\title{
A SUMMARY OF WATER-RESOURCES ACTIVITIES OF THE U.S. GEOLOGICAL SURVEY IN IOWA, FISCAL YEAR 1992
}

Compiled by Richard A. Karsten

\section{U.S. GEOLOGICAL SURVEY}

Open-File Report 92-154

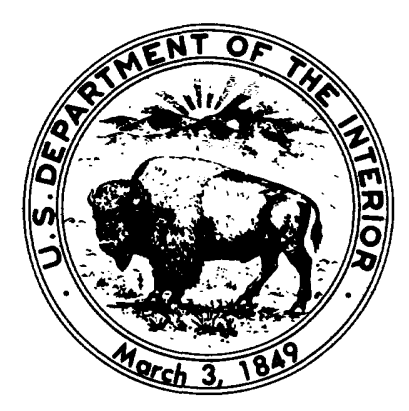

Iowa City, Iowa

1992 


\section{U.S. DEPARTMENT OF THE INTERIOR}

MANUEL LUJAN, JR., Secretary

\section{U.S. GEOLOGICAL SURVEY}<smiles>[CH]1CC1</smiles>

Dallas L. Peck, Director

For additional information write to:

Copies of this report can be purchased from:

District Chief

U.S. Geological Survey

Rm. 269, Federal Building

400 South Clinton Street

Iowa City, Iowa 52244
U.S. Geological Survey

Open-File Reports

Earth Science Information Center

Box 25425

Denver, Colorado 80225 


\section{CONTENTS}

Page

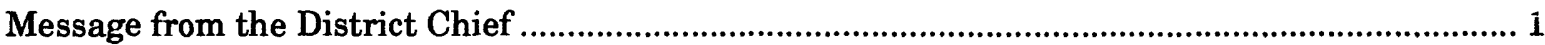

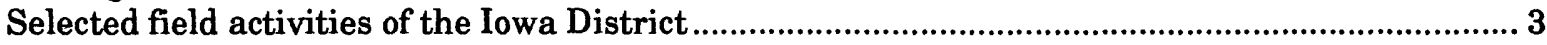

Origin and mission of the U.S. Geological Survey ........................................................................ 5

Mission and program of the Water Resources Division ................................................................. 6

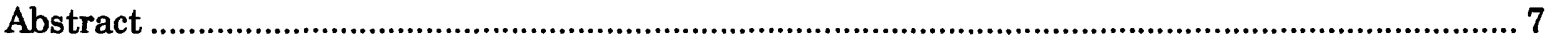

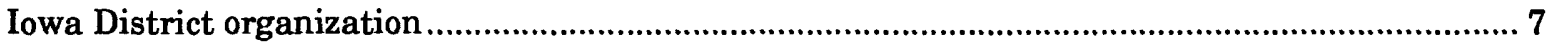

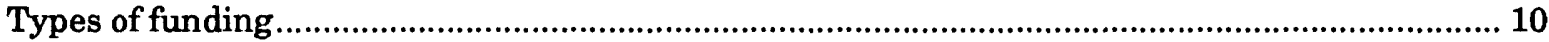

Where to obtain U.S. Geological Survey publications................................................................... 10

Hydrologic data-collection networks and programs................................................................... 13

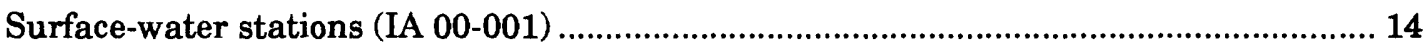

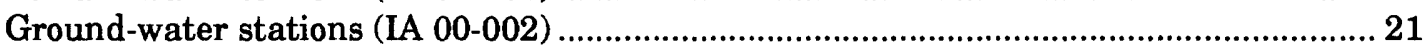

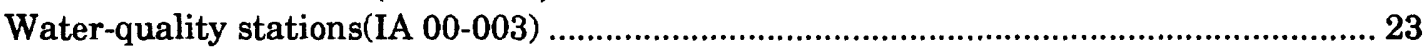

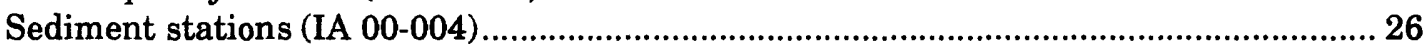

Iowa precipitation monitoring for the National trends network (IA 84-005) .................... 27

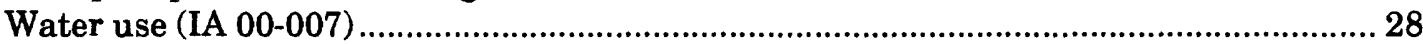

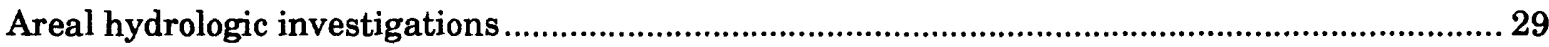

Flood information at selected bridge and culvert sites (IA 66-006) ....................................30

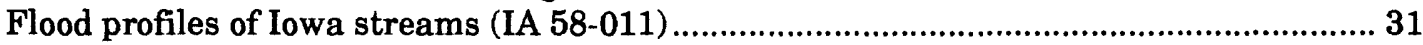

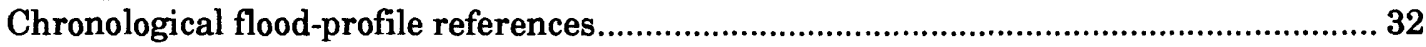

Iowa ground-water-quality monitoring program (IA 83-047) ........................................ 34

Des Moines Air National Guard Base, installation restoration program (IA 84-

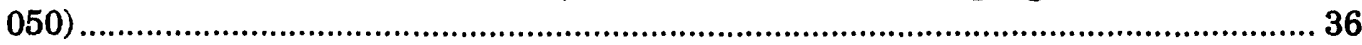

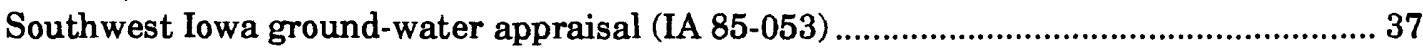

An accounting of pesticides in soil and ground water at selected sites in the Iowa

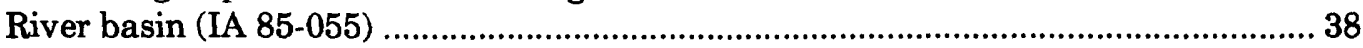

Side-looking airborne radar as a reconnaissance tool for the delineation of

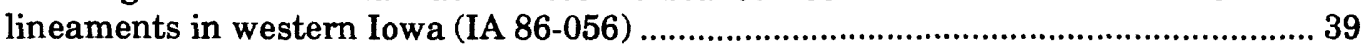

Evaluation of factors affecting the occurrence of agricultural chemicals in water resources in the central Midwest (IA 87-057) ........................................................... 40

Hydrologic analysis of water quality and the flow system in the Big Spring Basin, Clayton County, Iowa (IA 87-058)

Movement of nonpoint-source agricultural chemicals by the interaction of ground water and surface water in an alluvial aquifer (IA 88-061) ..................................... 44

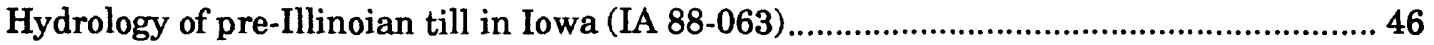

Estimating design flood discharges for Iowa using drainage-basin and channel-

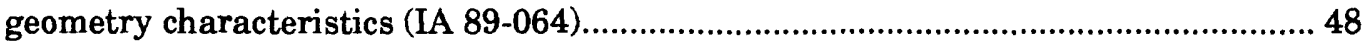

Analysis of the ground-water flow system, geochemistry, and underseepage in the vicinity of the Red Rock Dam near Pella, Iowa (IA 89-065) ...................................... 50

Water-flow processes and related agricultural chemical loadings in the Walnut Creek watershed near Ames, Iowa (IA 91-067) .................................................... 52

Agricultural chemicals in a water-supply reservoir in south-central Iowa (IA 91068)

Occurrence and flux of inert pesticide ingredients in shallow ground water (IA 91069).

Determination of storm-water runoff quality in Davenport, Iowa (IA 91-070) ....................56 


\section{CONTENTS}

Areal hydrologic investigations--Continued

Effect of land-use changes on stream sediment in the Sny Magill watershed, Clayton County, Iowa (IA 92-072)

Investigation of scour susceptibility at bridges in Iowa (IA 92-071) ................................ 58

Selected references

\section{ILLUSTRATIONS}

Figure 1. Diagram showing Iowa District organization.................................................. 8

2. Map showing location of Water Resources Division offices and areas assigned to field headquarters in Iowa ...............................................................9

3. Diagram showing distribution of funding of the U.S. Geological Survey in Iowa, fiscal year 1991

Figures 4-8.--Maps showing:

4. Location of continuous-record surface-water gaging stations .............................. 15

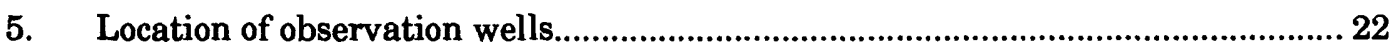

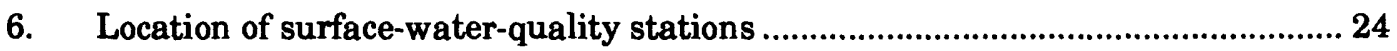

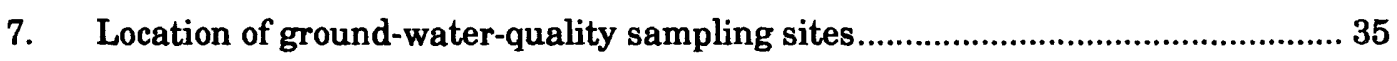

8. Area of focus for interagency research into the occurrence of agricultural chemicals in the water resources of the central Midwest

Figures 9-11.--Graphs showing:

9. Weekly precipitation, mean daily specific conductance, daily mean discharge, daily mean water temperature, and median $\mathrm{pH}$ at Big Spring, Clayton County, Iowa, water year 1990.

10. Cross-sectional distribution of atrazine in Cedar River alluvium near Cedar Rapids, Iowa, February through April 1990.

11. Daily mean water level in continuously monitored observation well EI-19, April-September 1990, near Cedar Rapids, Iowa.

Figures 12-13.--Maps showing:

12. Red Rock Dam site with observation wells near Pella, Iowa.

13. Walnut Creek watershed in parts of Boone and Story Counties near Ames, Iowa. 


\section{TABLES}

\section{Page}

Table 1. Agencies supporting water-resources investigations in Iowa during fiscal year 1992

2. Daily discharge and surface-water-quality stations operated by the Iowa District during fiscal year 1992

3. Discontinued surface-water gaging stations in Iowa .......................................... 19

4. Discontinued surface-water-quality stations in Iowa ..........................................24 


\section{CONVERSION FACTORS, ABBREVIATIONS, AND VERTICAL DATUM}

Multiply

inch (in.)

foot $(\mathrm{ft})$

mile (mi)

acre

acre

square mile $\left(\mathrm{mi}^{2}\right)$

cubic foot per second $\left(\mathrm{ft}^{3} / \mathrm{s}\right)$

ton per acre (ton/acre)

degree Fahrenheit $\left({ }^{\circ} \mathrm{F}\right)$
By

To obtain

Length

25.40

0.3048

1.609

Area

4,047

0.4047

2.590

Flow

0.02832

Mass

0.0002241

Temperature

(1) millimeter

meter

kilometer

square meter

hectare

square kilometer

cubic meter per second

megagram per square meter

degree Celsius $\left({ }^{\circ} \mathrm{C}\right)$

$1{ }^{\circ} \mathrm{C}=5 / 9\left({ }^{\circ} \mathrm{F}-32\right)$.

$$
{ }^{\circ} \mathrm{F}=9 / 5\left({ }^{\circ} \mathrm{C}\right)+32 \text {. }
$$

Sea level: In this report, "sea level" refers to the National Geodetic Vertical Datum of 1929--a geodetic datum derived from a general adjustment of the first-order level nets of the United States and Canada, formerly called Sea Level Datum of 1929.

The use of firm or trade names in this report is for identification purposes only and does not constitute endorsement by the U.S. Geological Survey. 


\section{MESSAGE FROM THE DISTRICT CHIEF}

The collection of hydrologic data and the investigation and assessment of the quantity, quality, and use of surface- and ground-water resources are major components of the mission of the Water Resources Division, U.S. Geological Survey. To accomplish this mission the Iowa District is organized into a district office and two field offices. These offices are geographically located in the State to provide access and response to hydrologic events and to maintain liaison with cooperating State and Federal agencies. The Iowa District technical staff is organized into two major sections: (1) the Hydrologic Surveillance Section, which maintains the systematic hydrologic data programs for the State and (2) the Hydrologic Studies Section, which investigates and assesses the quantity, quality, and use of the State's water resources.

The Hydrologic Surveillance Section maintains a network of hydrologic data-collection sites and compiles hydrologic data collected from these sites for public distribution. These hydrologic data include records of: (1) stage and discharge of principal rivers and tributaries; (2) chemistry and suspended-sediment concentration of selected rivers; (3) levels and quality of principal aquifers; (4) precipitation chemistry; and (5) surface- and ground-water use. Data from this network are compiled and entered in the Survey's National Water Information System data base, located in Reston, Virginia, and are published annually in the report series "Water Resources Data--Iowa."

The Iowa District has made substantial progress in developing a real-time hydrologic data base. Surface- and ground-water data at selected sites are transmitted to the Iowa District central hydrologic data base at regular intervals using satellite or ground data-relay systems. This information is accessible to scientists or water managers to assess current hydrologic conditions.

The investigation and assessment of the surface- and ground-water resources of Iowa is accomplished through a series of diversified projects by the Hydrologic Studies Section. Each project is managed by a designated project chief who is responsible for managing the investigative aspects of the project, maintaining a project budget, and providing public access to the findings of the project.

New projects planned for the Iowa District during fiscal 1992-93 include studies of:

- The Effects of Land-Use Changes on Stream Sediment in the Sny Magill Watershed

- Herbicide Degradation Processes in Small Streams

- Pier Scour Susceptibility at Bridges in Iowa

- Channel Aggradation and Degradation on Selected Main-Stem Rivers in Iowa.

The effects of land-use practices on water resources and the effects of chemical fertilizers and pesticides on surface- and ground-water quality is a growing public concern in Iowa. The Iowa District is presently conducting several State and Federally supported projects relating the effects of agricultural chemical use on the water resources of the State. An important part of the effort is an interagency research project presently being conducted at the Iowa Management System Evaluation Area (MSEA). The Iowa MSEA is a collaborative effort by the U.S. Department of Agriculture, the U.S. Geological Survey, the U.S. Environmental Protection Agency, the Iowa Department of Natural Resources, the University of Iowa Hygienic Laboratory, and Iowa State University. As part of this cooperative program, the lowa project will quantify the levels of nitrates and pesticides and their movement in soils according to climate, crops, and varying management practices. Research teams are assessing the impact of Iowa's current farming systems. These solutions will be arrived at through: (1) extensive monitoring of surface and ground water; (2) comparison of farming systems on water quality; and (3) evaluation of new and improved farming systems for their environmental and economic impact. Assessing the impact of these contaminants on surface- and ground-water supplies and analyzing the processes of chemical transport through the hydrologic systems represents both a challenge and an opportunity for the Iowa District for the 1990's. 
Utilizing the energy and interests of the Iowa District staff, the Iowa District can continue to assist cooperating State, Federal, and local agencies by providing accurate and timely hydrologic information. I look forward to continuing partnerships that will provide the basis for the beneficial use and management of Iowa's water resources.

N.B. Melcher

District Chief Iowa City, Iowa 


\section{SELECTED FIELD ACTIVITIES OF THE IOWA DISTRICT}

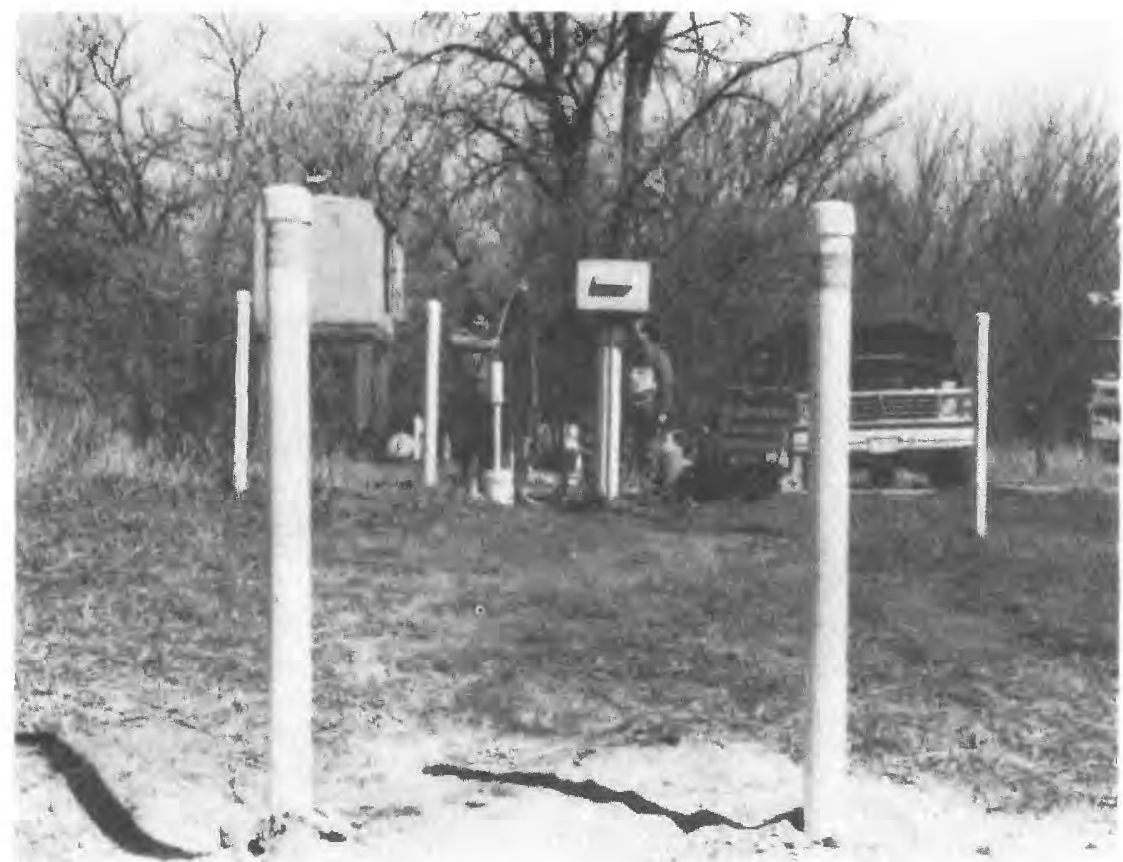

U.S. Geological Survey personnel collecting ground-water samples from the alluvium along the Cedar River in Linn County.

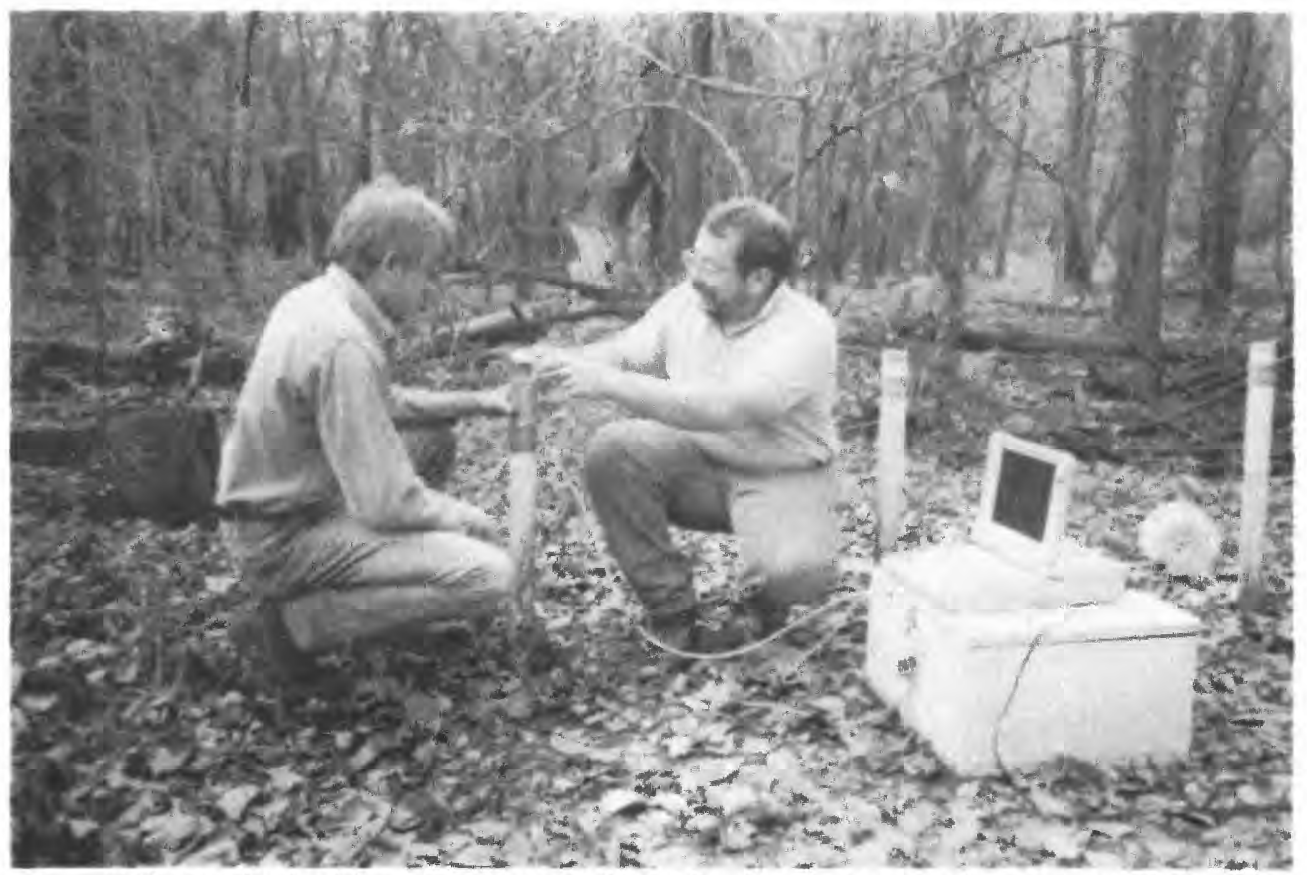

U.S. Geological Survey personnel conducting a slug test in the alluvium along the Cedar River in Linn County. 
The gaging station on Sny Magill Creek near Clayton in Clayton County.

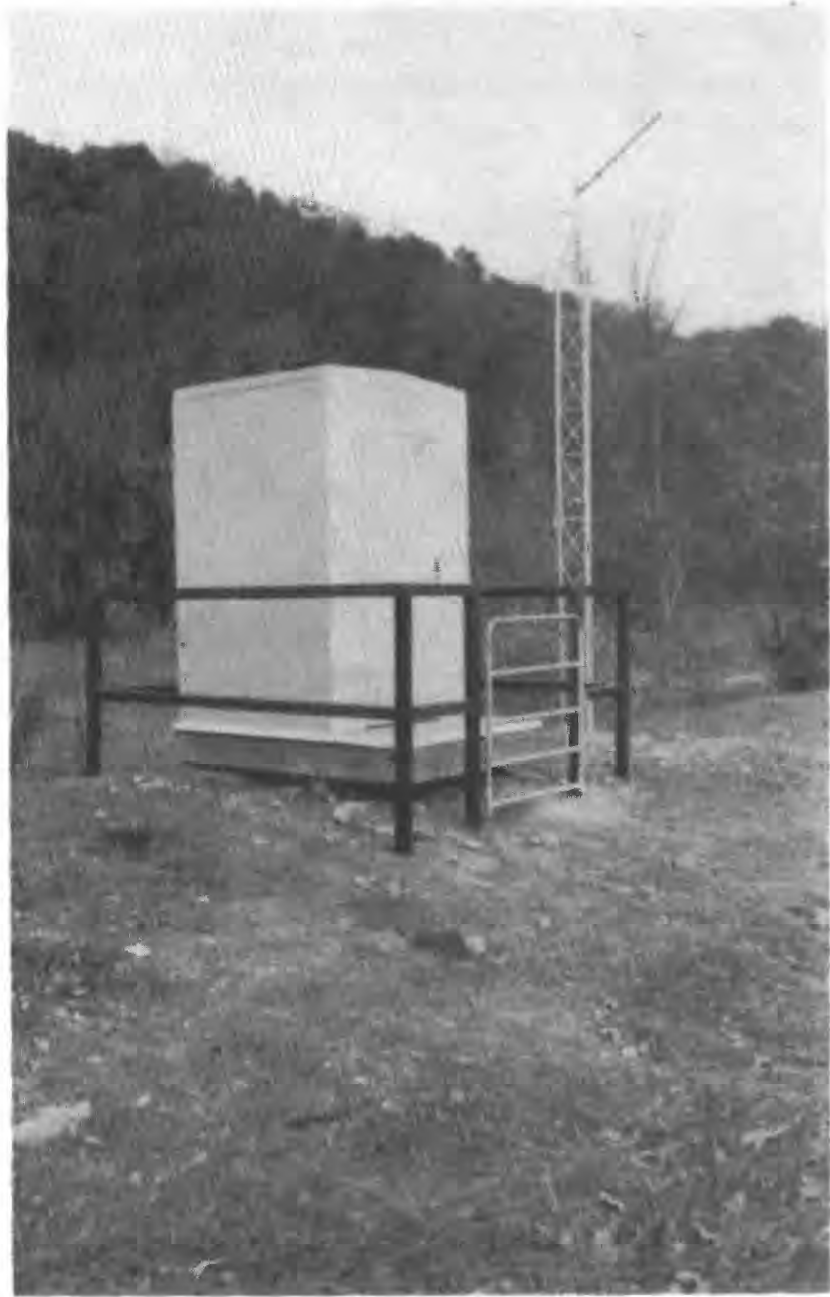

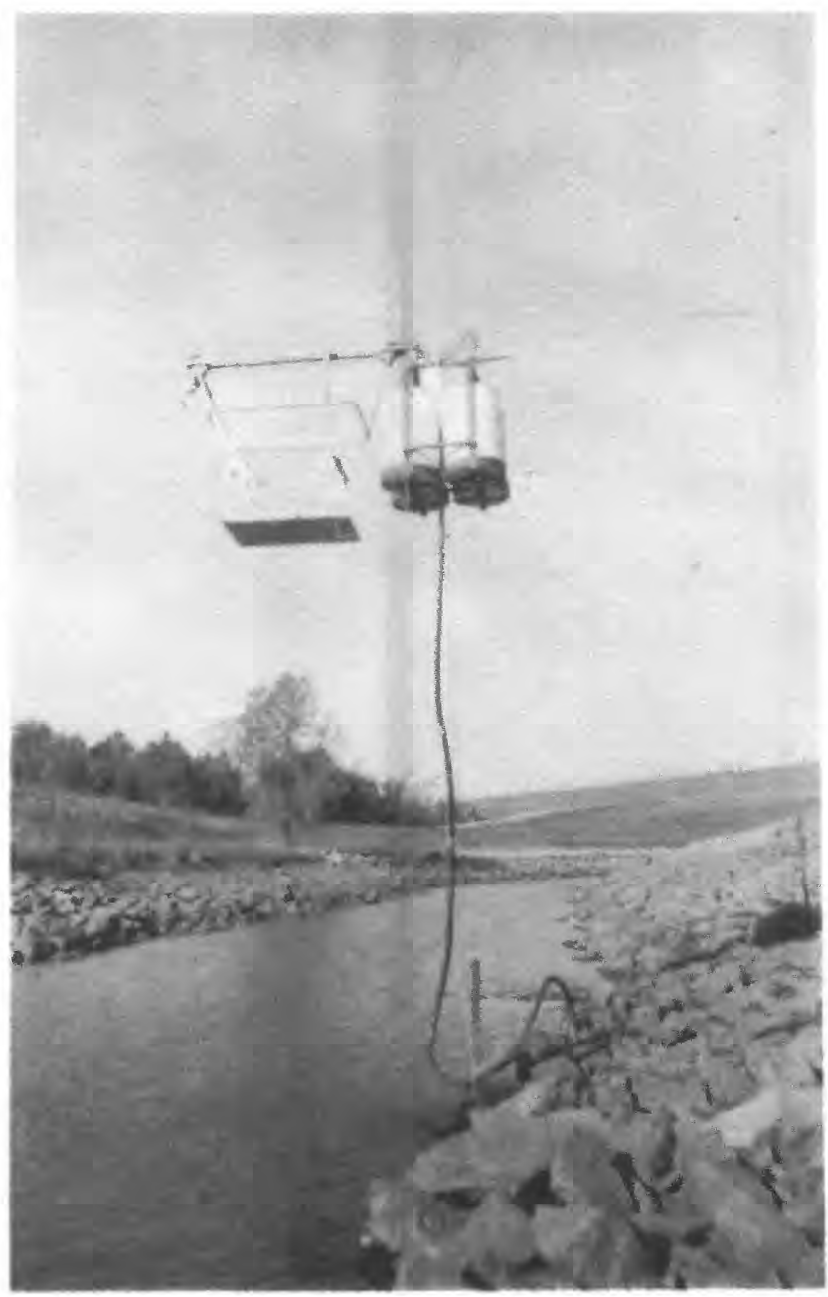

A cableway deadload tester on the Chariton River below the Rathbun Reservoir in Appanoose County. 


\section{ORIGIN AND MISSION OF THE U.S. GEOLOGICAL SURVEY}

The U.S. Geological Survey (USGS) was established by an act of Congress on March 3, 1879, to provide a permanent Federal agency to conduct the systematic and scientific classification of the public lands, and examination of the geological structure, mineral resources, and products of national domain. An integral part of that original mission includes publishing and disseminating the Earth-science information needed to understand, to plan the use of, and to manage the Nation's energy, land, mineral, and water resources.

Since 1879 , the research and fact-finding role of the USGS has grown and been modified to meet the changing needs of the Nation it serves. As part of that evolution, the USGS has become the Federal Government's largest Earth-science research agency, the Nation's largest civilian mapmaking agency, the primary source of data on the Nation's surface- and ground-water resources, and the employer of the largest number of professional Earth scientists. Today's programs serve a diversity of needs and users. Programs include:

- Conducting detailed assessments of the energy and mineral potential of the Nation's land and offshore areas.

o Investigating and issuing warnings of earthquakes, volcanic eruptions, landslides, and other geologic and hydrologic hazards.

- Conducting research on the geologic structure of the Nation.

o Studying the geologic features, structure, processes, and history of the other planets of our solar system.

- Craducting topographic surveys of the Nation and preparing topographic and thematic maps and related cartographic products.

- Developing and producing digital cartographic data bases and products.

- Collecting data on a routine basis to determine the quantity, quality, and use of surface and ground water.

- Conducting water-resource appraisals to describe the consequences of alternative plans for developing land and water resources.

o Conducting research in hydraulics and hydrology, and coordinating all Federal water-data acquisition.

- Using remotely sensed data to develop new cartographic, geologic, and hydrologic research techniques for natural resources planning and management.

o Providing Earth-science information through an extensive publications program and a network of public-access points.

Along with its continuing commitment to meet the growing and changing Earth-science needs of the Nation, the USGS remain s dedicated to its original mission to collect, analyze, interpret, publish, and disseminate information about the natural resources of the Nation--providing "Earth Science in the Public Service." 


\section{MISSION AND PROGRAM OF THE WATER RESOURCES DIVISION}

The mission of the Water Resources Division is to provide the hydrologic information and understanding needed for the optimum utilization and management of the Nation's water resources for the overall benefit of the people of the United States.

This is accomplished, in large part, through cooperation with other Federal and non-Federal agencies by:

- Collecting, on a systematic basis, data needed for the continuing determination and evaluation of the quantity, quality, and use of the Nation's water resources.

- Conducting analytical and interpretive water-resources appraisals describing the occurrence, availability, and the physical, chemical, and biological characteristics of surface and ground water.

- Conducting basic and problem-oriented research in hydraulics, hydrology, and related fields of science to improve the scientific basis for investigations and measurement techniques and to understand hydrologic systems to predict their response to stress, either natural or human-induced.

- Disseminating water data and the results of investigations and research through reports, maps, computerized information services, and other forms of public releases.

- Coordinating the activities of Federal agencies in the acquisition of water data for streams, lakes, reservoirs, estuaries, and ground water.

- Providing scientific and technical assistance in hydrologic fields to other Federal, State, and local agencies, to licensees of the Federal Energy Regulatory Commission, and to international agencies on behalf of the Department of State. 


\title{
A SUMMARY OF WATER-RESOURCES ACTIVITIES OF THE U.S. GEOLOGICAL SURVEY IN IOWA, FISCAL YEAR 1992

\author{
Compiled by \\ Richard A. Karsten
}

\begin{abstract}
Water-resources activities of the U.S. Geological Survey in lowa consist of collecting hydrologic data and conducting interpretive studies. Hydrologic investigations in lowa are made through three basic types of projects: (1) hydrologic data-collection programs; (2) local or areal studies; and (3) statewide or regional investigations. These projects are funded through cooperative joint-funding agreements with Federal, State, and local agencies and direct Federal funds. The data and the results of the interpretive studies are published or released by either the U.S. Geological Survey or by cooperating agencies. This report describes: (1) the hydrologic data-collection programs; (2) the local or areal hydrologic investigations; and (3) statewide or regional studies conducted by the U.S. Geological Survey in lowa during fiscal year 1992 and provides a list of selected water-resources references for lowa.
\end{abstract}

\section{IOWA DISTRICT ORGANIZATION}

The Iowa District of the U.S. Geological Survey, Water Resources Division, consists of two operating sections (the Hydrologic Surveillance Section and the Hydrologic Studies Section), two support units (the Administrative Services Section and the Computer Services Section), and three field offices (fig. 1). Personnel are based at the District Office in Iowa City and at field headquarters in Council Bluffs, Fort Dodge, and Iowa City (fig. 2). The District operates with guidance from the Office of Midwest Programs in Lawrence, Kansas, the Central Regional Office in Denver, Colorado, and the National Headquarters in Reston, Virginia.

Inquires regarding projects described in this report, ground-water data, and water-quality data may be directed to the District Office listed below. Requests for streamflow data should be directed to the District Office or the field headquarters nearest the area of concern (fig. 2).

\section{Iowa District Office}

\author{
N.B. Melcher \\ District Chief \\ Water Resources Division \\ U.S. Geological Survey \\ 269 Federal Building \\ P.O. Box 1230 \\ Iowa City, Iowa 52244 \\ Telephone (319) 337-4191
}




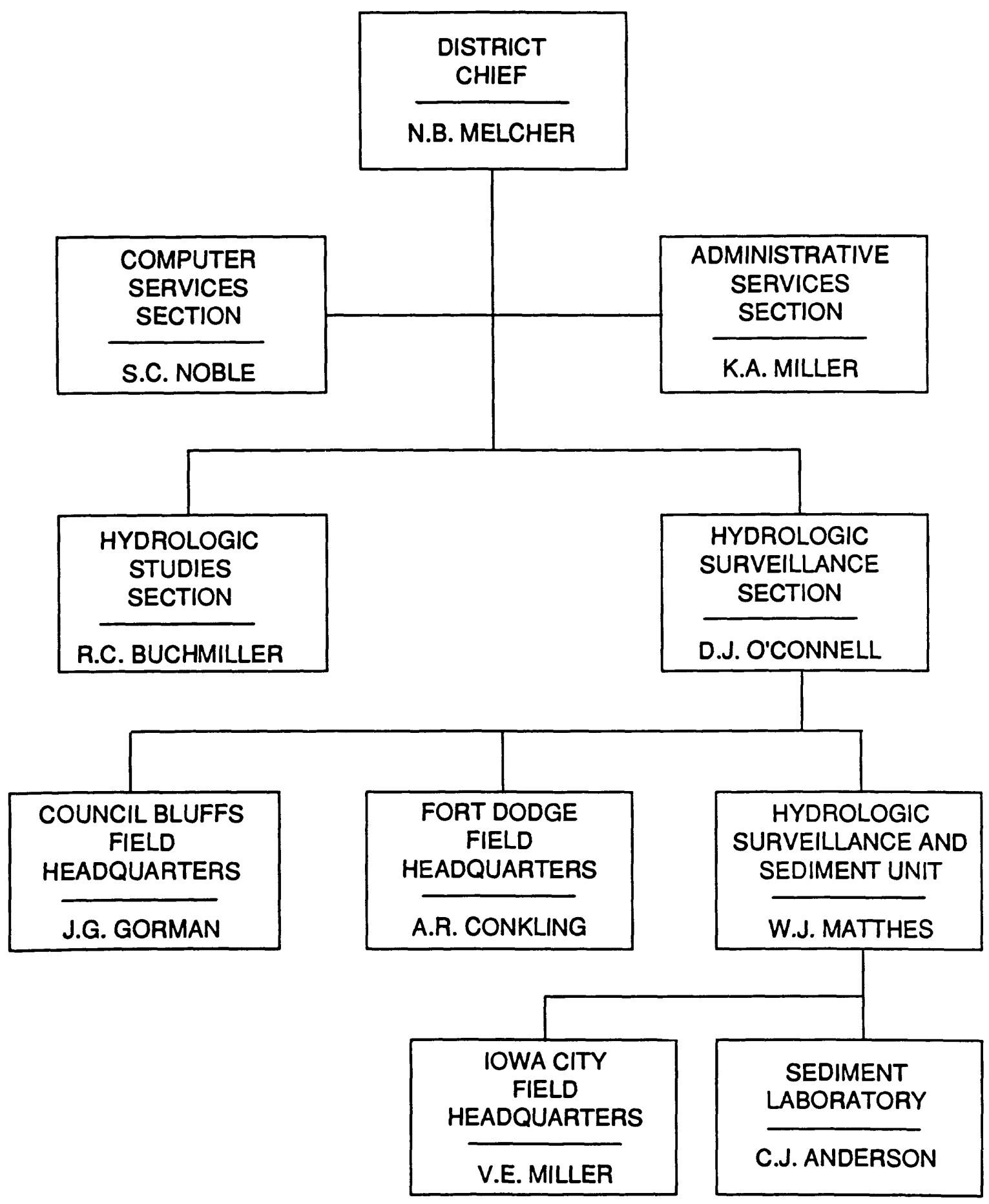

Flgure 1. Lowa District organization. 


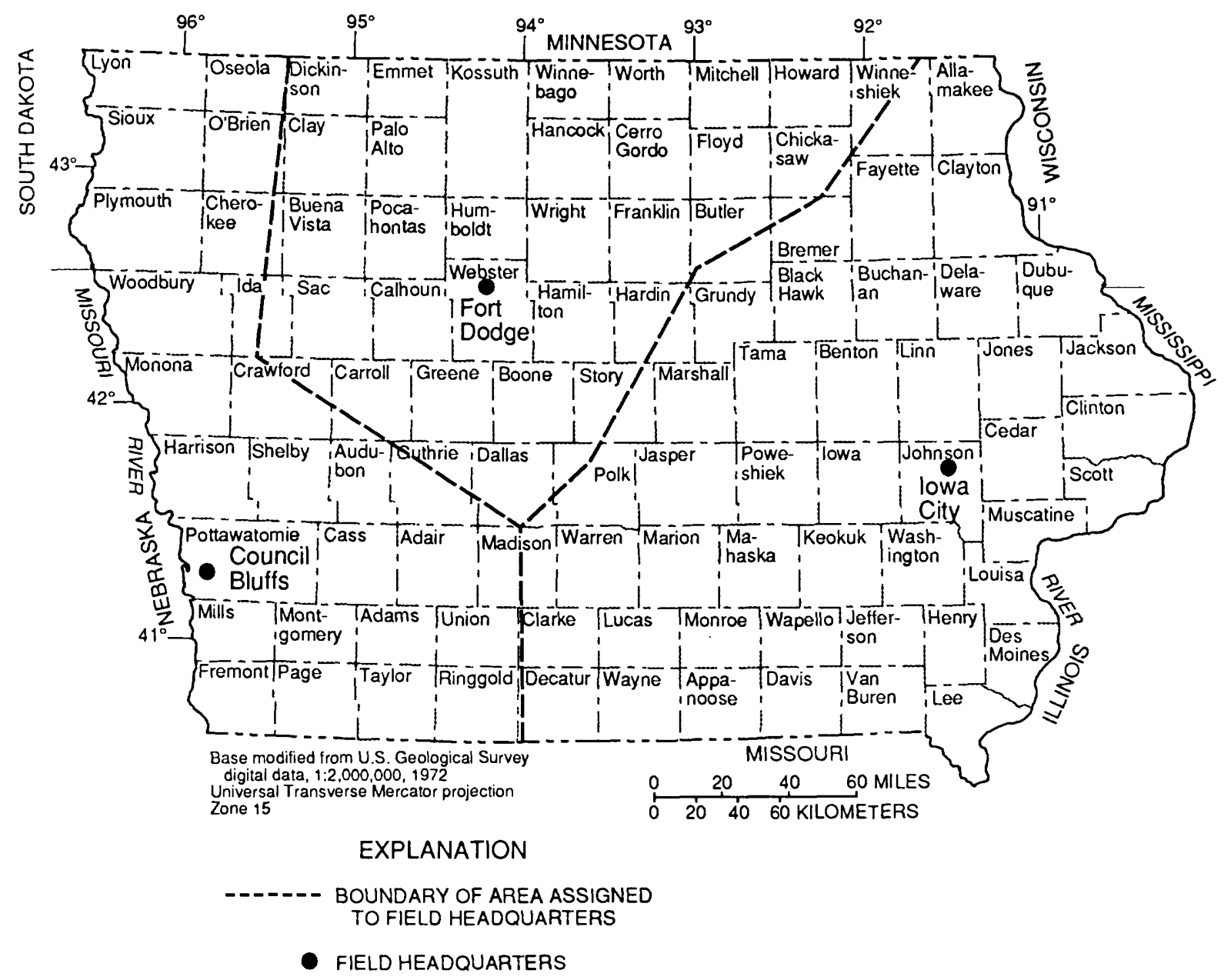

Figure 2. Location of Water Resources Division offices and areas assigned to field headquarters in lowa.

Field Headquarters

J.G. Gorman
Supervisory Hydrologic Technician
Water Resources Division
U.S. Geological Survey
250 Federal Building
P.O. Box 917
Council Bluffs, Iowa 51502
Telephone: (712) 323-8024

A.R. Conkling

Lead Hydrologic Technician

Water Resources Division

U.S. Geological Survey

456 Federal Building

P.O. Box 693

Fort Dodge, Iowa 50501

Telephone: (515) 576-4571

V.E. Miller

Lead Hydrologic Technician

Water Resources Division

U.S. Geological Survey

269 Federal Building

P.O. Box 1240

Iowa City, Iowa 52244

Telephone: (319) 337-4191 


\title{
TYPES OF FUNDING
}

The hydrologic investigations and data-collection efforts in the Iowa District are supported by services and joint funding provided by State and local agencies (table 1) on a 50-50 basis with Federal monies (cooperative program); by funds transferred from other Federal agencies (OFA), such as the U.S. Army Corps of Engineers (OFA program); and by funds appropriated directly to the U.S. Geological Survey (Federal program). In fiscal year 1991, the financial support for these programs in Iowa was $\$ 3,513,816$, which was distributed as shown in figure 3 .

\section{WHERE TO OBTAIN \\ U.S. GEOLOGICAL SURVEY PUBLICATIONS}

Current publications are described in a monthly pamphlet, "New Publications of the Geological Survey," which may be obtained from:

\author{
U.S. Geological Survey \\ Books and Open-File Reports \\ Federal Center, Building 810 \\ Box 25425 \\ Denver, Colorado 80225
}

Professional Papers, Bulletins, Water-Supply Papers, Techniques of Water-Resources Investigations, Circulars, Earthquake Bulletins, and popular leaflets, pamphlets, and booklets may be purchased from the above address. Additional information is given in "Guide to Obtaining Information from the U.S. Geological Survey, 1989," U.S. Geological Survey Circular 900, which is available at no cost from the above address. Reports and maps of flood-prone areas for Iowa are available for inspection at the Iowa District Office. Other map information is available from:

\author{
U.S. Geological Survey \\ Map Distribution \\ Federal Center, Building 810 \\ Box 25286 \\ Denver, Colorado 80225
}

Requests for miscellaneous water information and information on programs in other states may be referred to:

\author{
Water Resources Division \\ U.S. Geological Survey \\ Mail Stop $\mathbf{4 4 0}$ \\ 12201 Sunrise Valley Drive \\ Reston, Virginia 22092
}


Table 1. Agencies supporting water-resources investigations in Iowa during fiscal year 1992

\section{STATE AGENCIES:}

Iowa Department of Natural Resources

Iowa Department of Transportation

Highway Division

Highway Research Board

Iowa State University

Department of Agricultural Engineering and

Biosystems Engineering

Iowa State Water Resources Research Institute

The University of Iowa

Department of Preventive Medicine and

Environmental Health

Hygienic Laboratory

Institute of Hydraulic Research

\section{LOCAL AGENCIES:}

Carroll County Auditor

Carroll County Soil and Water Conservation District

City of Ames

City of Cedar Rapids

City of Charles City

City of Clear Lake

City of Davenport

City of Denison

City of Des Moines

City of Des Moines Water Works

City of Fort Dodge

City of Iowa City

City of Marshalltown

City of Sioux City

City of Waterloo

City of Waterloo Sewage Treatment Plant

Union Electric Company, Keokuk

West Central Iowa Rural Water Association, Manning

\section{FEDERAL AGENCIES:}

U.S. Department of Agriculture

U.S. Department of the Army

U.S. Army Corps of Engineers

Kansas City District

Omaha District

Rock Island District

St. Paul District

U.S. Department of Defense

Air Force Center for Environmental Excellence

U.S. Department of the Interior

U.S. Geological Survey

Water Resources Division (Federal Program)

U.S. Environmental Protection Agency 


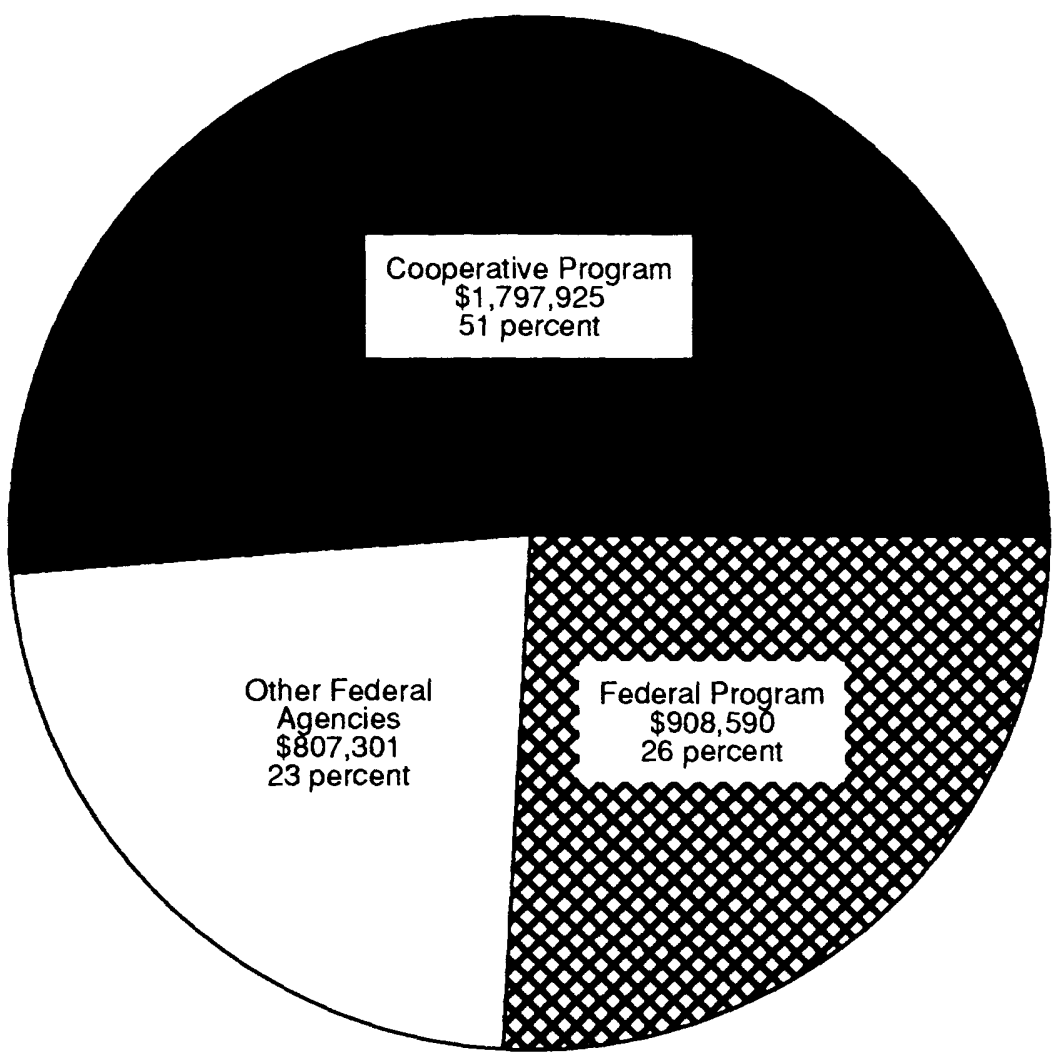

Figure 3. Distribution of funding of the U.S. Geological Survey in lowa, fiscal year 1991.

The U.S. Geological Survey's National Center maintains a library with an extensive Earth-sciences collection. Local libraries may obtain books, periodicals, and maps through the interlibrary loan system by writing to:

\author{
U.S. Geological Survey Library \\ 12201 Sunrise Valley Drive \\ Reston, Virginia 22092
}


HYDROLOGIC DATA-COLLECTION NETWORKS AND PROGRAMS 


\section{SURFACE-WATER STATIONS}

(IA 00-001)

PERIOD OF PROJECT: Continuous since 1902

PROJECT CHIEF: D.J. O’Connell

STUDY AREA: Statewide

COOPERATING AGENCIES: Iowa Department of

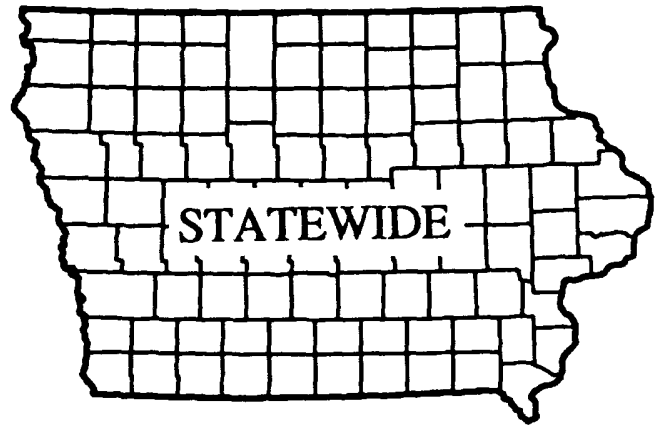

Natural Resources (Environmental

Protection Division, Geological Survey Bureau), U.S. Army Corps of

Engineers, the University of Iowa (Institute of Hydraulic Research and

Department of Preventive Medicine and Environmental Health), Iowa

Department of Transportation (Highway Division, Highway Research

Advisory Board), Iowa State University (Department of Agricultural

Engineering and Biosystems Engineering and the Iowa State Water

Resources Research Institute), City of Cedar Rapids, City of

Davenport, City of Des Moines, City of Fort Dodge, Union Electric

Company, Des Moines Water Works, Waterloo Sewage Treatment

Plant, the University of Iowa, West Central Iowa Rural Water

Association, City of Charles City, City of Clear Lake, City of Denison, City of Iowa City, City of Marshalltown, City of Sioux City, and City of Waterloo.

NEED FOR STUDY: Streamflow supplies water for many uses, including domestic, commercial, and industrial uses; irrigation of crops; dilution and transport for removal of wastes; energy for hydroelectric-power generation; transport channels for commerce; and a medium for recreation. Records of streamflow are the basic data used in developing reliable surface-water supplies because the records provide information on the availability of streamflow and its variability in time and space. Streamflow, when it occurs in excess, can create a hazard--floods cause extensive damage and hardship. Records of floods obtained at streamflow-gaging stations serve as the basis for the design of bridges, culverts, dams, and flood-control reservoirs, and for flood-plain delineation and flood-warning systems. Streamflow records are used in the planning and design of surface-water related projects, and they are used in the management or operation of such projects after the projects have been completed.

OBJECTIVES: To collect surface-water data sufficient to satisfy the needs for current uses, such as: (1) assessment of water resources; (2) operation of reservoirs or industries; (3) forecasting; (4) pollution control and disposal of wastes; (5) discharge data to accompany water-quality measurements; (6) contract and legal requirements; and (7) research or special studies. To collect the data necessary for analytical studies to define, for any location, the statistical properties and trends in discharge or elevation of streams, lakes, and reservoirs. 
PROGRESS: Surface-water data were collected, compiled, and published. The annual data report, "Water Resources Data-Iowa, Water Year 1990" was completed during 1991. The data network consists of 119 daily streamflow-gaging stations, 5 lakes and stream-stage stations, and 4 reservoir-contents stations. Data for floods in Iowa were compiled and will be published in the Survey's "Summary of Floods in the United States During 1991." Data for 86 of the streamflow-gaging stations are transmitted to the Iowa District's central computer using a real-time telemetry network. A complete list of active stations is given in table 2, locations are shown in figure 4, and a list of discontinued gaging stations is given in table 3 .

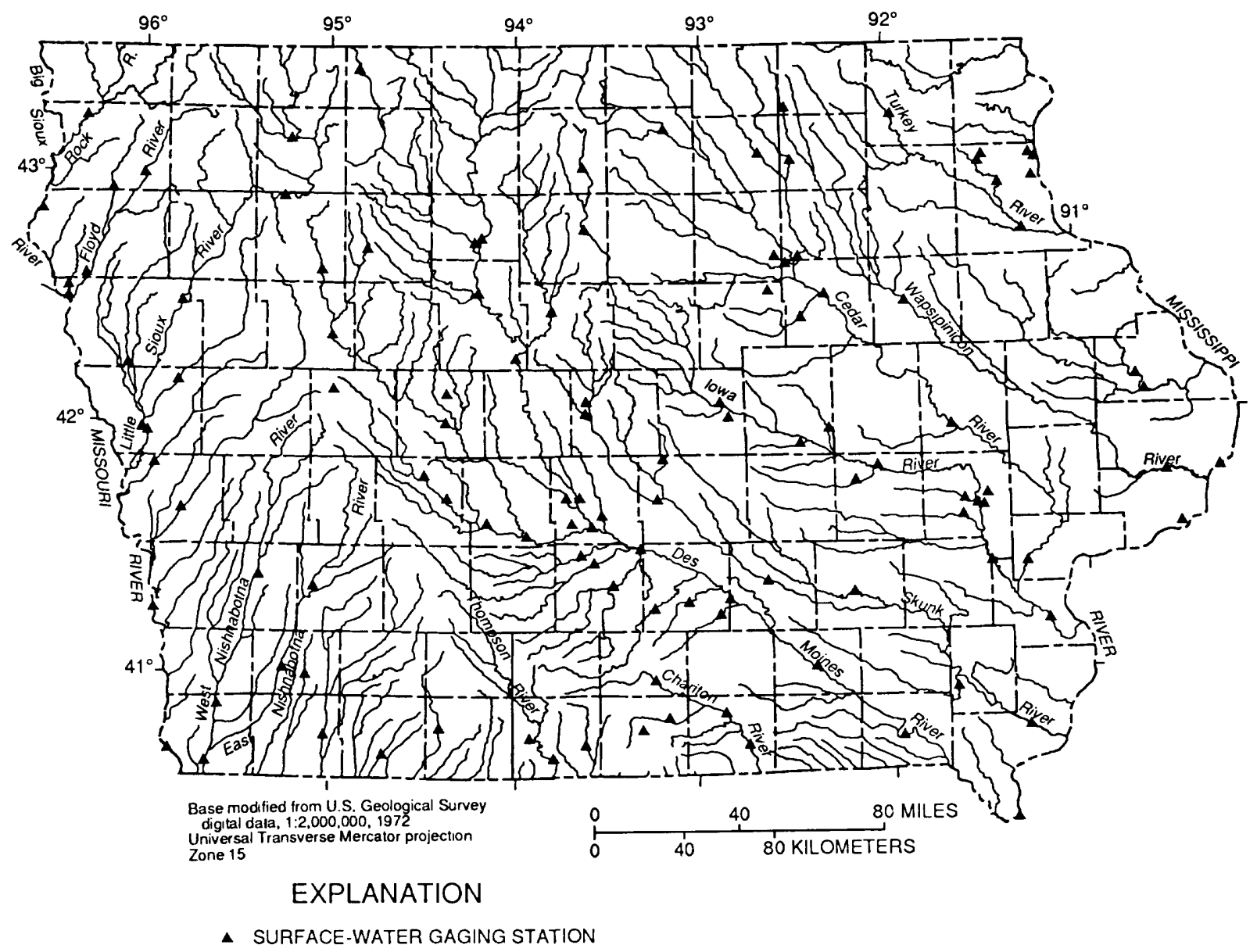

Figure 4. Location of continuous-record surface-water gaging stations. 
Table 2. Daily discharge and surface-water-quality stations operated by the Iowa District during fiscal year 1992

[Letter after station name designates type of data: (d) discharge,(c) chemical, (m) microbiological, (t) water temperature, (s) sediment]

\begin{tabular}{|c|c|c|c|}
\hline Station name & Station number & $\begin{array}{l}\text { Drainage area } \\
\text { (square miles) }\end{array}$ & Period of record \\
\hline Upper Iowa River near Dorchester (d) & 05388250 & 770 & $1936-92$ \\
\hline Bloody Run Creek near Marquette (dts) & 05389400 & 1992 & \\
\hline Mississippi River at McGregor (dts) & 05389500 & 67,500 & 1936-92 \\
\hline Sny Magill Creek near Clayton (dts) & 05411400 & 27.6 & 1992 \\
\hline Turkey River at Spillville (d) & 05411600 & 177 & $1956-70,1977-92$ \\
\hline Silver Creek near Luana (d) & 05412060 & 4.39 & 1986-92 \\
\hline Unnamed Creek near Luana (d) & 05412070 & 1.15 & 1886-92 \\
\hline Roberts Creek above Saint Olaf (d) & 05412100 & 70.7 & $1957-77,1986-92$ \\
\hline Turkey River at Garber (d) & 05412500 & 1,545 & $\begin{array}{l}1913-16,1919-27 \\
1929-30,1932-92\end{array}$ \\
\hline North Fork Maquoketa River at Fulton (d) & 05418450 & 516 & $1977-92$ \\
\hline Maquoketa River near Maquoketa (d) & 05418500 & 1,553 & 1913-92 \\
\hline Mississippi River at Clinton (d) & 05420500 & 85,600 & 1873-1992 \\
\hline Wapsipinicon River near Elma (d) & 05420560 & 95.2 & $1958-92$ \\
\hline Wapsipinicon River at Independence (d) & 05421000 & 1,048 & 1933-92 \\
\hline Wapsipinicon River near De Witt (d) & 05422000 & 2,330 & $1934-92$ \\
\hline Crow Creek at Bettendorf (d) & 05422470 & 17.8 & $1977-92$ \\
\hline East Branch Iowa River near Klemme (d) & 05449000 & 133 & $1948-76,1977-92$ \\
\hline Iowa River near Rowan (d) & 05449500 & 429 & $1940-76,1977-92$ \\
\hline Iowa River at Marshalltown (dts) & 05451500 & 1,564 & $\begin{array}{l}\text { 1902-03, 1914-27, } \\
1932-92\end{array}$ \\
\hline Timber Creek near Marshalltown (d) & 05451700 & 118 & $1949-92$ \\
\hline Richland Creek near Haven (d) & 05451900 & 56.1 & $1949-92$ \\
\hline Salt Creek near Elberon (d) & 05452000 & 201 & $1945-92$ \\
\hline Walnut Creek near Hartwick (d) & 05452200 & 70.9 & 1949-92 \\
\hline Big Bear Creek at Ladora (d) & 05453000 & 189 & $1945-92$ \\
\hline Iowa River at Iowa City (d) & 05454500 & 3,271 & $1903-92$ \\
\hline Iowa River at Marengo (d) & 05453100 & 2,794 & $1956-92$ \\
\hline Coralville Lake near Coralville & 05453510 & 3,115 & $1958-92$ \\
\hline Rapid Creek near Iowa City (d) & 05454000 & 23.5 & $1937-92$ \\
\hline Clear Creek near Coralville (d) & 05454300 & 98.1 & $1952-92$ \\
\hline South Branch Ralston Creek at Iowa City (d) & 05455010 & 2.94 & $1963-92$ \\
\hline Old Mans Creek near Iowa City (d) & 05455100 & 201 & $1950-77,1984-92$ \\
\hline English River at Kalona (d) & 05455500 & 573 & 1939-92 \\
\hline Iowa River near Lone Tree (d) & 05455700 & 4,293 & $1956-92$ \\
\hline Cedar River at Charles City (d) & 05457700 & 1,054 & 1964-92 \\
\hline Little Cedar River near Ionia (d) & 05458000 & 306 & $1954-92$ \\
\hline Cedar River at Janesville (d) & 05458500 & 1,661 & $\begin{array}{l}1904-06,1914-27, \\
1932-42,1945-92\end{array}$ \\
\hline West Fork Cedar River at Finchford (d) & 05458900 & 846 & $1945-92$ \\
\hline Winnebago River at Mason City (d) & 05459500 & 526 & $1932-92$ \\
\hline Clear Lake at Clear Lake & 05460000 & 22.6 & 1933-92 \\
\hline Shell Rock River at Shell Rock (d) & 05462000 & 1,746 & $1953-92$ \\
\hline Beaver Creek at New Hartford (d) & 05463000 & 347 & $1945-92$ \\
\hline Cedar River at Cedar Falls (c) & 05463050 & 4,734 & $\begin{array}{l}1975-79,1984-85 \\
1986-92\end{array}$ \\
\hline Black Hawk Creek at Hudson (d) & 05463500 & 303 & $1952-92$ \\
\hline
\end{tabular}


Table 2. Daily discharge and surface-water-quality stations operated by the Iowa District during fiscal year 1992--Continued

\begin{tabular}{|c|c|c|c|}
\hline Station name & Station number & $\begin{array}{l}\text { Drainage area } \\
\text { (square miles) }\end{array}$ & Period of record \\
\hline Cedar River at Waterloo (d) & 05464000 & 5,146 & $1940-92$ \\
\hline Cedar River at Cedar Rapids (d) & 05464500 & 6,510 & $1902-92$ \\
\hline Cedar River near Conesville (d) & 05465000 & 7,785 & 1939-92 \\
\hline Iowa River at Wapello (dcmts) & 05465500 & 12,499 & $1914-92$ \\
\hline South Skunk River (head of Skunk River) near Ames (d) & 05470000 & 315 & $1920-27,1932-92$ \\
\hline Squaw Creek at Ames (d) & 05470500 & 204 & 1919-27, 1965-92 \\
\hline South Skunk below Squaw Creek near Ames (d) & 05471000 & 556 & $1952-79,1992$ \\
\hline South Skunk River at Colfax (d) & 05471050 & 803 & $1985-92$ \\
\hline Indian Creek near Mingo (d) & 05471200 & 276 & $1958-75,1985-92$ \\
\hline South Skunk River near Oskaloosa (d) & 05471500 & 1,635 & $1945-92$ \\
\hline North Skunk River near Sigourney (d) & 05472500 & 730 & 1945-92 \\
\hline Cedar Creek near Oakland Mills (d) & 05473400 & 530 & $1957-77,1977-92$ \\
\hline Skunk River at Augusta (dcmts) & 05474000 & 4,303 & $1913-14,1914-92$ \\
\hline Mississippi River at Keokuk (d) & 05474500 & 119,000 & 1878-1992 \\
\hline Des Moines River at Estherville (d) & 05476500 & 1,372 & $1951-92$ \\
\hline Des Moines River at Humboldt (d) & 05476750 & 2,256 & $1964-92$ \\
\hline East Fork Des Moines River at Dakota City (d) & 05479000 & 1,308 & $1940-92$ \\
\hline Des Moines River at Fort Dodge (d) & 05480500 & 4,190 & $\begin{array}{l}1905-06,1913-27, \\
1946-92\end{array}$ \\
\hline Boone River near Webster City (d) & 05481000 & 844 & $1940-92$ \\
\hline Des Moines River near Stratford (d) & 05481300 & 5,452 & $1920-92$ \\
\hline Saylorville Lake near Saylorville & 05481630 & 5,823 & $1977-92$ \\
\hline Des Moines River near Saylorville (dts) & 05481650 & 5,841 & $1961-92$ \\
\hline Beaver Creek near Grimes (d) & 05481950 & 358 & $1960-92$ \\
\hline North Raccoon River near Newell (d) & 05482135 & 233 & $1982-92$ \\
\hline Big Cedar Creek near Varina (d) & 05482170 & 80 & $1959-92$ \\
\hline North Raccoon River near Sac City (d) & 05482300 & 700 & 1958-92 \\
\hline Black Hawk Lake at Lake View & 05482315 & 23.3 & $1970-75,1978-92$ \\
\hline North Raccoon River near Jefferson (d) & 05482500 & 1,619 & $1940-92$ \\
\hline East Fork Hardin Creek near Churdan (d) & 05483000 & 24 & $1952-92$ \\
\hline Hazelbrush Creek near Maple River (dts) & 05483343 & 9.22 & $1991-92$ \\
\hline Middle Raccoon River near Bayard (d) & 05483450 & 375 & $1979-92$ \\
\hline Lake Panorama at Panora & 05483470 & 433 & 1979-92 \\
\hline Middle Raccoon River at Panora (d) & 05483600 & 440 & 1958-92 \\
\hline South Raccoon River at Redfield (d) & 05484000 & 994 & $1940-92$ \\
\hline Raccoon River at Van Meter (dc) & 05484500 & 3,441 & 1915-92 \\
\hline Walnut Creek at Des Moines (d) & 05484800 & 78.4 & 1971-92 \\
\hline Des Moines River below Raccoon River at Des Moines"(d) & 05485500 & 9,879 & $1940-92$ \\
\hline Fourmile Creek at Des Moines (d) & 05485640 & 92.7 & $1971-92$ \\
\hline North River near Norwalk (d) & 05486000 & 349 & $1940-92$ \\
\hline Middle River near Indianola (d) & 05486590 & 503 & $1940-92$ \\
\hline South River near Ackworth (d) & 05487470 & 460 & $1940-92$ \\
\hline Des Moines River near Runnells(d) & 05487500 & 11,655 & $1985-92$ \\
\hline White Breast Creek near Dallas (d) & 05487980 & 342 & $1962-92$ \\
\hline Lake Red Rock near Pella & 05488100 & 12,323 & 1963-92 \\
\hline English Creek near Knoxville (d) & 05488200 & 90.1 & 1985-92 \\
\hline Des Moines River near Tracy (d) & 05488500 & 12,479 & 1920-92 \\
\hline Cedar Creek near Bussey (d) & 05489000 & 374 & $1947-92$ \\
\hline Des Moines River at Ottumwa (d) & 05489500 & 13,374 & $1917-92$ \\
\hline
\end{tabular}


Table 2. Daily discharge and surface-water-quality stations operated by the Iowa District during fiscal year 1992 --Continued

\begin{tabular}{|c|c|c|c|}
\hline Station name & Station number & $\begin{array}{l}\text { Drainage area } \\
\text { (square miles) }\end{array}$ & Period of record \\
\hline Des Moines River at Keosauqua (d) & 05490500 & 14,038 & $1903-06,1910-92$ \\
\hline Rock River near Rock Valley (d) & 06483500 & 1,592 & $1948-92$ \\
\hline Big Sioux River at Akron (d) & 06485500 & 8,424 & $1928-92$ \\
\hline Missouri River at Sioux City (ds) & 06486000 & 314,600 & 1897-1992 \\
\hline Perry Creek at 38th Street, Sioux City (d) & 06600000 & 65.1 & $1945-69,1981-92$ \\
\hline Floyd River at Alton (d) & 06600100 & 268 & $1955-92$ \\
\hline West Branch Floyd River near Struble (d) & 06600300 & 180 & 1955-92 \\
\hline Floyd River at James (d) & 06600500 & 886 & $1934-92$ \\
\hline Missouri River at Decatur, Nebraska (d) & 06601200 & 316,200 & $1987-92$ \\
\hline \multicolumn{2}{|c|}{ West Fork ditch (head of Monana-Harrison ditch) at Hornick (d)06602020 } & 403 & $1939-69,1974-92$ \\
\hline Monona-Harrison ditch near Turin (d) & 06602400 & 900 & 1939-92 \\
\hline Spirit Lake near Orleans & 06604000 & 75.6 & $1933-75,1990-92$ \\
\hline West Okoboji Lake at Lakeside Laboratory near Milford & 06604200 & 125 & 1933-92 \\
\hline Ocheyedan River near Spencer (d) & 06605000 & 426 & $1977-92$ \\
\hline Little Sioux River at Linn Grove (d) & 06605850 & 1,548 & 1972-92 \\
\hline Little Sioux River at Correctionville (d) & 06606600 & 2,500 & $\begin{array}{l}1918-25,1928-32, \\
1936-92\end{array}$ \\
\hline Maple River at Mapleton (d) & 06607200 & 669 & $1941-92$ \\
\hline Little Sioux River near Turin (d) & 06607500 & 3,526 & $1958-92$ \\
\hline Soldier River at Pisgah (d) & 06608500 & 407 & $1940-92$ \\
\hline Boyer River at Logan (d) & 06609500 & 871 & $1918-25,1937-92$ \\
\hline Missouri River at Omaha, Nebraska (ds) & 06610000 & 322,800 & $1928-92$ \\
\hline Missouri River at Nebraska City, Nebraska (ds) & 06807000 & 410,000 & 1929-92 \\
\hline West Nishnabotna River at Hancock (d) & 06807410 & 609 & $1959-92$ \\
\hline West Nishnabotna River at Randolph (d) & 06808500 & 1,326 & 1948-92 \\
\hline East Nishnabotna River near Atlantic (d) & 06809210 & 436 & $1960-92$ \\
\hline East Nishnabotna River at Red Oak (d) & 06809500 & 894 & $1918-25,1936-92$ \\
\hline Nishnabotna River above Hamburg (dcmts) & 06810000 & 2,806 & $1922-23,1928-92$ \\
\hline Tarkio River at Stanton (d) & 06811840 & 49.3 & $1957-92$ \\
\hline Missouri River at Rulo, Nebraska (d) & 06813500 & 414,900 & 1949-92 \\
\hline Nodaway River at Clarinda (dets) & 06817000 & 762 & $1918-25,1936-92$ \\
\hline Platte River near Diagonal (d) & 06818750 & 217 & 1968-92 \\
\hline East Fork One Hundred and Two River near Bedford (d) & 06819185 & 85.4 & 1983-92 \\
\hline Elk Creek near Decatur City (dcmts) & 06897950 & $\mathbf{5 2 . 5}$ & $1967-92$ \\
\hline Thompson River at Davis City (d) & 06898000 & 701 & $1918-25,1941-92$ \\
\hline Weldon River near Loon (d) & 06898400 & 104 & 1958-92 \\
\hline Chariton River near Chariton (d) & 06903400 & 182 & $1965-92$ \\
\hline Corydon Lake at spillway at Corydon (dc) & 06903677 & 2.5 & 1991-92 \\
\hline South Fork Chariton River near Promise City (d) & 06903700 & 168 & $1967-92$ \\
\hline Rathbun Lake near Rathbun & 06903880 & 549 & 1969-92 \\
\hline Chariton River near Rathbun (d) & 06903900 & 549 & $1956-92$ \\
\hline Chariton River near Moulton (d) & 06904010 & 740 & $1979-92$ \\
\hline
\end{tabular}


[The following surface-water gaging stations have been discontinued in Iowa. Continuous daily streamflow records were collected and published for the period of record shown for each station]

\begin{tabular}{|c|c|c|c|}
\hline Station name & Station number & $\begin{array}{l}\text { Drainage area } \\
\text { (square miles) }\end{array}$ & Period of record \\
\hline Upper Iowa River at Decorah & 05387500 & 511 & $1952-83$ \\
\hline Upper Iowa River near Decorah & 05388000 & 568 & $\begin{array}{l}\text { 1913-14, 1919-27, } \\
1933-51\end{array}$ \\
\hline Paint Creek at Waterville & 05388500 & 42.8 & $1952-73$ \\
\hline Yellow River at Ion & 05389000 & 221 & $1934-51$ \\
\hline Mississippi River at Clayton & 05411500 & 9,200 & $1930-36$ \\
\hline Turkey River at Elkader & 05412000 & 891 & $1932-42$ \\
\hline Little Maquoketa River near Durango & 05414500 & 130 & $1934-82$ \\
\hline Maquoketa River near Manchester & 05417000 & 305 & $1933-73$ \\
\hline Maquoketa River near Delhi & 05417500 & 347 & $1933-40$ \\
\hline Bear Creek near Monmouth & 05417700 & 61.3 & $1957-76$ \\
\hline $\begin{array}{l}\text { Maquoketa River above North Fork Maquoketa River } \\
\text { near Maquoketa }\end{array}$ & 05418000 & 938 & $1913-14$ \\
\hline Wapsipinicon River at Stone City & 05421500 & 1,324 & $1903-14$ \\
\hline Crow Creek at Eldridge & 05422420 & 2.20 & $1977-82$ \\
\hline Creek at Mt. Joy & 05422450 & 6.90 & $1977-82$ \\
\hline Pine Creek at Muscatine & 05448150 & 38.9 & $1975-82$ \\
\hline Eagle Lake inlet near Britt & 05448285 & 3.83 & $1975-80$ \\
\hline Eagle Lake outlet near Britt & 05448290 & 11.3 & $1975-80$ \\
\hline West Branch (West Fork) Iowa River near Klemme & 05448500 & 112 & 1948-58 \\
\hline Iowa River near Iowa Falls & 05450000 & 665 & $1911-14$ \\
\hline Upper Pine Lake at Eldora & 05450500 & 14.9 & 1936-70 \\
\hline Lower Pine Lake at Eldora & 05451000 & 15.9 & $1936-70$ \\
\hline Iowa River near Belle Plaine & 05452500 & 2,455 & 1939-59 \\
\hline Lake Macbride near Solon & 05453500 & 27.0 & $1936-71$ \\
\hline Ralston Creek at Iowa City & 05455000 & 3.01 & $1924-87$ \\
\hline Cedar River at Mitchell & 05457500 & 826 & $1933-42$ \\
\hline Shell Rock River near Northwood & 05459000 & 300 & $1945-86$ \\
\hline Shell Rock River at Marble Rock (Greene) & 05460500 & 1,318 & 1933-53 \\
\hline Shell Rock River at Greene & 05461000 & 1,357 & $1933-42$ \\
\hline Shell Rock River near Clarksville & 05461500 & 1,626 & $1915-27,1932-34$ \\
\hline Fourmile Creek near Lincoln & 05464130 & 13.78 & $1962-67,1969-74$ \\
\hline Half Mile Creek near Gladbrook & 05464133 & 1.33 & $1962-67,1969-74$ \\
\hline Fourmile Creek near Traer & 05464137 & 19.51 & $1962-74,1975-80$ \\
\hline Prairie Creek at Fairfax & 05464640 & 178 & $1966-82$ \\
\hline South Skunk River below Squaw Creek near Ames & 05471000 & 556 & $1952-79$ \\
\hline Lake Keomah near Oskaloosa & 05472000 & 3.06 & 1936-71 \\
\hline Skunk River at Coppock & .05473000 & 2,916 & $1913-44$ \\
\hline Big Creek near Mount Pleasant & 05473500 & 106 & 1955-79 \\
\hline East Fork Des Moines River near Burt & 05478000 & 462 & 1971-74 \\
\hline East Fork Des Moines River near Hardy & 05478500 & 1,268 & $1940-54$ \\
\hline Des Moines River near Fort Dodge & 05479500 & 3,753 & $1911-13$ \\
\hline
\end{tabular}


Table 3. Discontinued surface-water gaging stations in Iowa--Continued

\begin{tabular}{|c|c|c|c|}
\hline Station name & Station number & $\begin{array}{l}\text { Drainage area } \\
\text { (square miles) }\end{array}$ & Period of record \\
\hline $\begin{array}{l}\text { Lizard Creek near Clare } \\
\text { Des Moines River near Boone } \\
\text { Des Moines River at Des Moines } \\
\text { Storm Lake at Storm Lake } \\
\text { Springbrook Lake near Guthrie Center }\end{array}$ & $\begin{array}{l}05480000 \\
05481500 \\
05482000 \\
05482140 \\
05483500\end{array}$ & $\begin{array}{c}257 \\
5,511 \\
6,245 \\
28.3 \\
5.18\end{array}$ & $\begin{array}{l}1940-82 \\
1920-68 \\
1905-06,1915-61 \\
1970-75 \\
1936-71\end{array}$ \\
\hline $\begin{array}{l}\text { Raccoon River at Des Moines } \\
\text { Lake Ahquabi near Indianola } \\
\text { White Breast Creek near Knoxville } \\
\text { Muchakinock Creek near Eddyville } \\
\text { Lake Wapello near Drakesville }\end{array}$ & $\begin{array}{l}05485000 \\
05487000 \\
05488000 \\
05489190 \\
05490000\end{array}$ & $\begin{array}{c}3,590 \\
4.93 \\
380 \\
70.2 \\
7.75\end{array}$ & $\begin{array}{l}1902-03 \\
1936-71 \\
1945-62 \\
1975-79 \\
1936-71\end{array}$ \\
\hline $\begin{array}{l}\text { Sugar Creek near Keokuk } \\
\text { Fox River at Bloomfield } \\
\text { Fox River at Cantril } \\
\text { Rock River at Rock Rapids } \\
\text { Dry Creek at Hawarden }\end{array}$ & $\begin{array}{l}05491000 \\
05494300 \\
05494500 \\
06483270 \\
06484000\end{array}$ & $\begin{array}{l}105 \\
87.7 \\
161 \\
788 \\
48.4\end{array}$ & $\begin{array}{l}1922-31,1958-73 \\
1957-73 \\
1940-61 \\
1959-74 \\
1948-69\end{array}$ \\
\hline $\begin{array}{l}\text { West Fork ditch at Holly Springs } \\
\text { Loon Creek near Orleans } \\
\text { Spirit Lake outlet at Orleans } \\
\text { Milford Creek at Milford } \\
\text { Little Sioux River at Spencer }\end{array}$ & $\begin{array}{l}06602000 \\
06603920 \\
06604100 \\
06604400 \\
06605100\end{array}$ & $\begin{array}{c}399 \\
31 \\
75.6 \\
146 \\
990\end{array}$ & $\begin{array}{l}1939-69 \\
1971-74 \\
1971-74 \\
1971-74 \\
1936-42\end{array}$ \\
\hline $\begin{array}{l}\text { Little Sioux River at Gillett Grove } \\
\text { Little Sioux River near Kennebeck } \\
\text { Odebolt Creek near Arthur } \\
\text { Maple River at Turin } \\
\text { Little Sioux River near Blencoe (Turin) }\end{array}$ & $\begin{array}{l}06605600 \\
06606700 \\
06607000 \\
06607300 \\
06607510\end{array}$ & $\begin{array}{c}1,334 \\
2,738 \\
39.3 \\
725 \\
4,470\end{array}$ & $\begin{array}{l}1958-73 \\
1939-69 \\
1957-75 \\
1939-41 \\
1939-42\end{array}$ \\
\hline $\begin{array}{l}\text { Steer Creek near Magnolia } \\
\text { Thompeon Creek near Woodbine } \\
\text { Willow Creek near Logan } \\
\text { Indian Creek at Council Blufif } \\
\text { Mosquito Creek near Earling }\end{array}$ & $\begin{array}{l}06609200 \\
06609590 \\
06609600 \\
06610500 \\
06610520\end{array}$ & $\begin{array}{c}9.26 \\
6.97 \\
129 \\
7.99 \\
32.0\end{array}$ & $\begin{array}{l}1963-69 \\
1963-69 \\
1972-75 \\
1954-76 \\
1965-79\end{array}$ \\
\hline $\begin{array}{l}\text { Waubonsie Creek near Bartlett } \\
\text { West Nishnabotna River at Harlan } \\
\text { Weat Nishnabotna River at (near) White Cloud } \\
\text { Mule Creek near Malvern } \\
\text { Spring Valley Creek near Tabor }\end{array}$ & $\begin{array}{l}06806000 \\
06807320 \\
06807500 \\
06808000 \\
06808200\end{array}$ & $\begin{array}{c}30.4 \\
316 \\
967 \\
10.6 \\
7.6\end{array}$ & $\begin{array}{l}1946-69 \\
1977-82 \\
1918-24 \\
1954-69 \\
1955-64\end{array}$ \\
\hline $\begin{array}{l}\text { Davids Creek near Hamlin } \\
\text { Tarkio River at Blanchard } \\
\text { West Nodaway River at Villisca } \\
\text { Honey Creek near Russell } \\
\text { Chariton River near Centerville }\end{array}$ & $\begin{array}{l}06809000 \\
06812000 \\
06816500 \\
06903500 \\
06904000\end{array}$ & $\begin{array}{l}26.0 \\
200 \\
342 \\
13.2 \\
708\end{array}$ & $\begin{array}{l}1952-73 \\
1934-40 \\
1918-25 \\
1952-62 \\
1938-59\end{array}$ \\
\hline
\end{tabular}


GROUND-WATER STATIONS

(IA 00-002)

PERIOD OF PROJECT: Continuous since 1939

PROJECT CHIEF: R.B. Lambert

STUDY AREA: Statewide

COOPERATING AGENCY: Iowa Department of Natural Resources (Geological Survey Bureau).

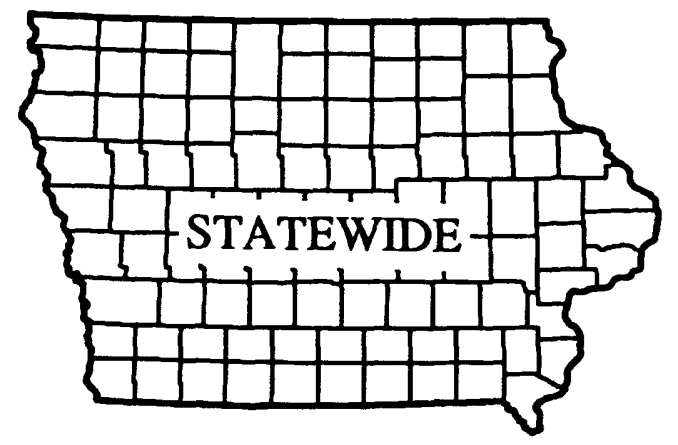

NEED FOR STUDY: A long-term regional ground-water data base is needed to evaluate the effects of natural and human-induced stresses on the principal ground-water systems in Iowa. Information from the long-term data base will provide baseline information for short-term aquifer studies, as well as documenting long-term trends in Iowa's ground-water supply. Information from the short-term and long-term data bases will be used to assess the ground-water resource, project future conditions of supply, address contamination concerns, and provide the information necessary to effectively manage the resource.

OBJECTIVES: The primary objectives of the Iowa ground-water-level monitoring network are to: (1) collect data documenting the change in ground-water storage through time in the principal aquifers; (2) provide both the long-term and short-term data necessary to assess and predict the response of hydrologic systems to natural climatic variations and human-induced stresses; (3) quantify the hydrologic characteristics of aquifers including transmissivity, hydraulic conductivity, and specific capacity; and (4) provide historical baseline data for aquifer studies.

PROGRESS: The ground-water-level monitoring network (fig. 5) has been redesigned and expanded to improve the areal distribution and aquifer coverage across Iowa. The current (1991) monitoring network has 202 observation wells. Three data components of the monitoring-network have been established to address the primary objectives of the project. These three data networks are: (1) hydrologic; (2) water-management; and (3) baseline data. The hydrologic network is designed to document the change in storage and define the areal extent of an aquifer. The water-management network provides information needed to assess and predict the effects of natural stresses and human activities on aquifer recharge and discharge. The baseline network collects information that documents long-term changes in climate and the effects of topography and geology on the climatic response. Historical water levels for the observation wells have been updated in the Survey's WATer data STOrage and REtrieval System (WATSTORE). Quality-assurance techniques have been implemented to ensure the integrity of the data. The annual data report, "Water Resources Data--Iowa, Water Year 1990" was published in May 1991 and included data for 185 observation wells. Monitoring data for the 1991 water year will be compiled and published in the annual data report. An open-file report "The Ground-Water-Level Monitoring Network in Iowa" is in preparation. 


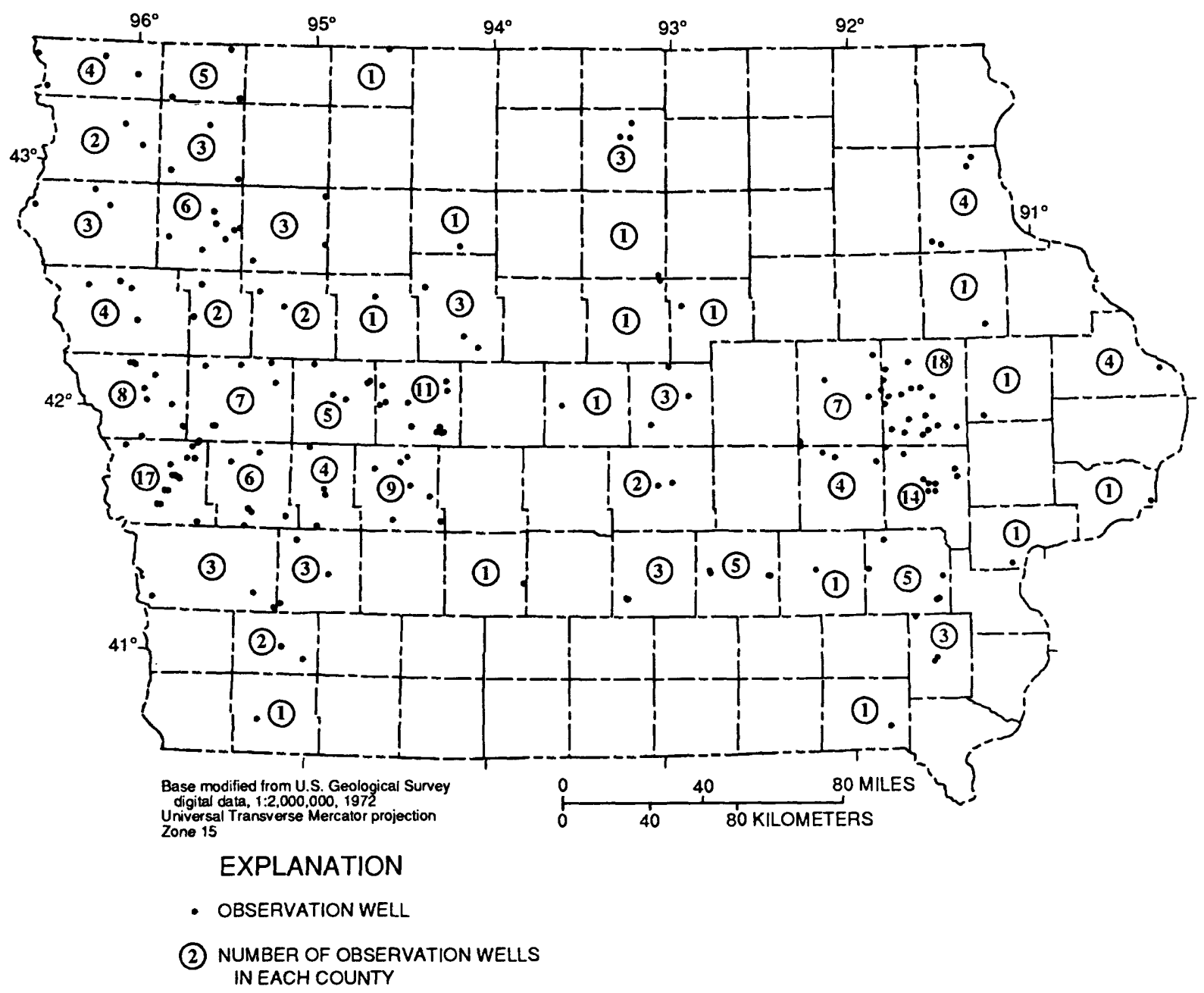

Flgure 5. Location of observation wells. 
WATER-QUALITY STATIONS

(IA 00-003)

PERIOD OF PROJECT: Continuous since 1906

PROJECT CHIEF: D.J. O’Connell

STUDY AREA: Statewide

COOPERATING AGENCY: U.S. Geological Survey

(Federal program).

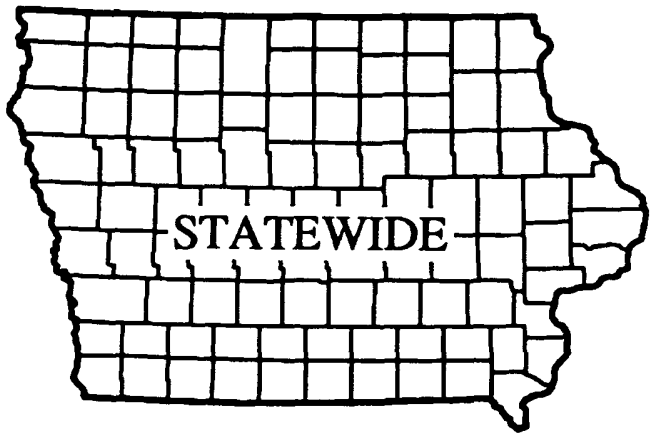

NEED FOR STUDY: Water-resource planning and water-quality assessment require a nationwide data base with standardized information for planning and assessment of water resources. Furthermore, the chemical and physical quality of the rivers and streams must be monitored and defined.

OBJECTIVES: To provide a national data base for water-quality information for Federal planning and action programs and to provide data for Federal management of interstate waters.

PROGRESS: Water-quality data were collected, compiled, and processed for publication in the annual data report for five National Stream-Quality Accounting Network (NASQAN) stations and one National Hydrologic Bench-Mark Station. Measurements of water temperature and specific conductance were obtained during discharge measurements at most surface-water gaging stations and published in the annual report. The annual data report, "Water Resources Data--Iowa, Water Year 1990" was delivered in May 1991. Station locations are shown in figure 6, and a list of discontinued surface-water-quality stations is given in table 4 . Sampling for verification of herbicides in nine streams in Iowa, as part of the Regional Program Verification Sampling for Herbicides in Upper Midwest Streams, was completed in 1990. Results of this study were published in the ASCE Irrigation and Drainage Proceedings 1991 as an article entitled "Geographic and Seasonal Distribution of Herbicides in Streams of the Midwestern United States" (Goolsby and others, 1991b). Sampling for herbicides in the Mississippi River near Clinton (fig. 6) began in April 1991 and will continue through the spring runoff season of 1992. This sampling is part of a program to study of the occurrence, distribution, and transport of agricultural chemicals in the Mississippi, Missouri, and Ohio River basins. 


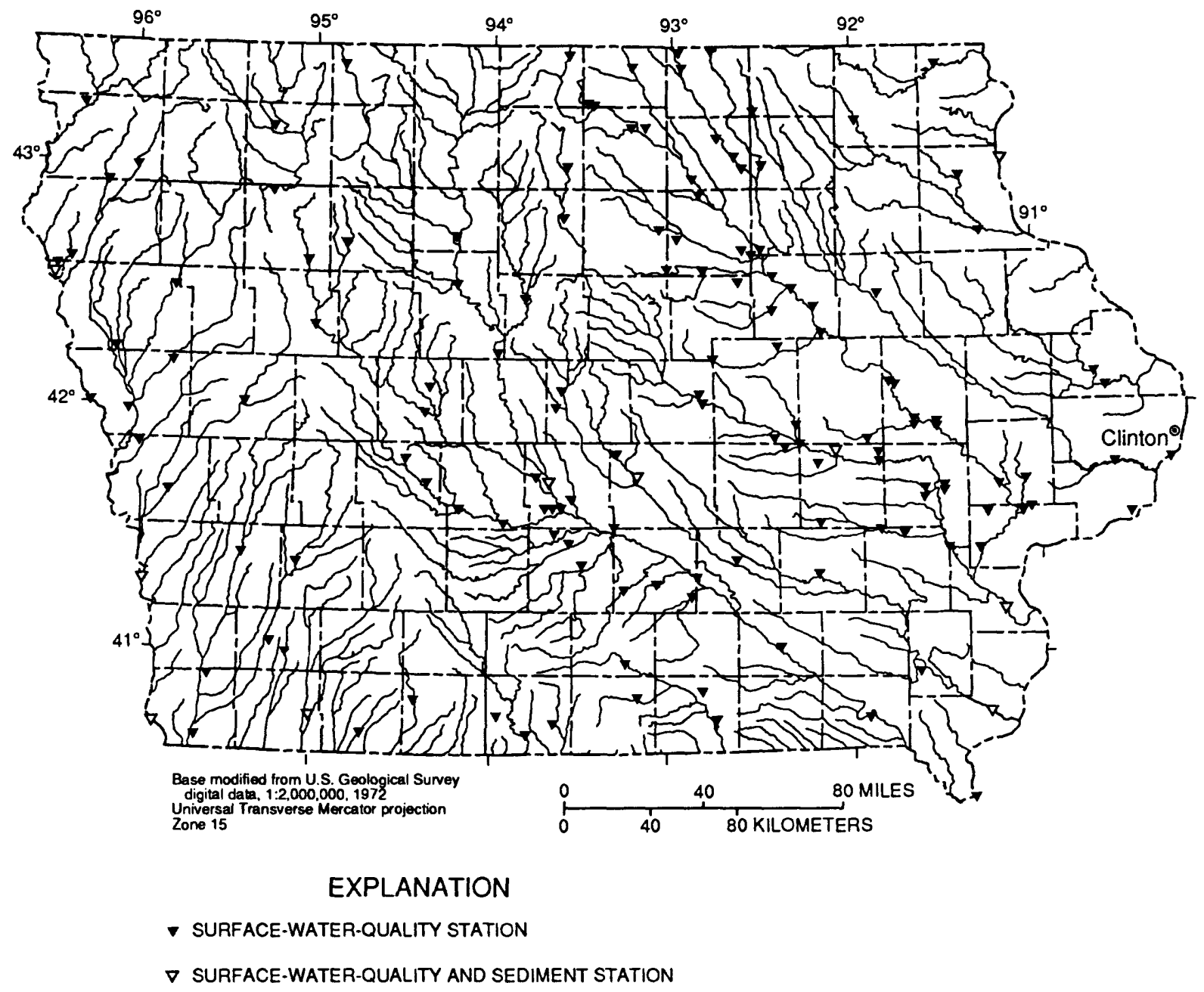

Figure 6. Location of surface-water-quality stations.

Table 4. Discontinued surface-water-quality stations in Iowa

[The following surface-water-quality stations have been discontinued in Iowa. Continuous daily records of water temperature or sediment and monthly or periodic samples of chemical quality were collected and published for the period of record shown for each station. An asterisk $\left(^{*}\right)$ in the type of record column indicates that periodic data are available for that water-quality constituent subsequent to the period of daily record]

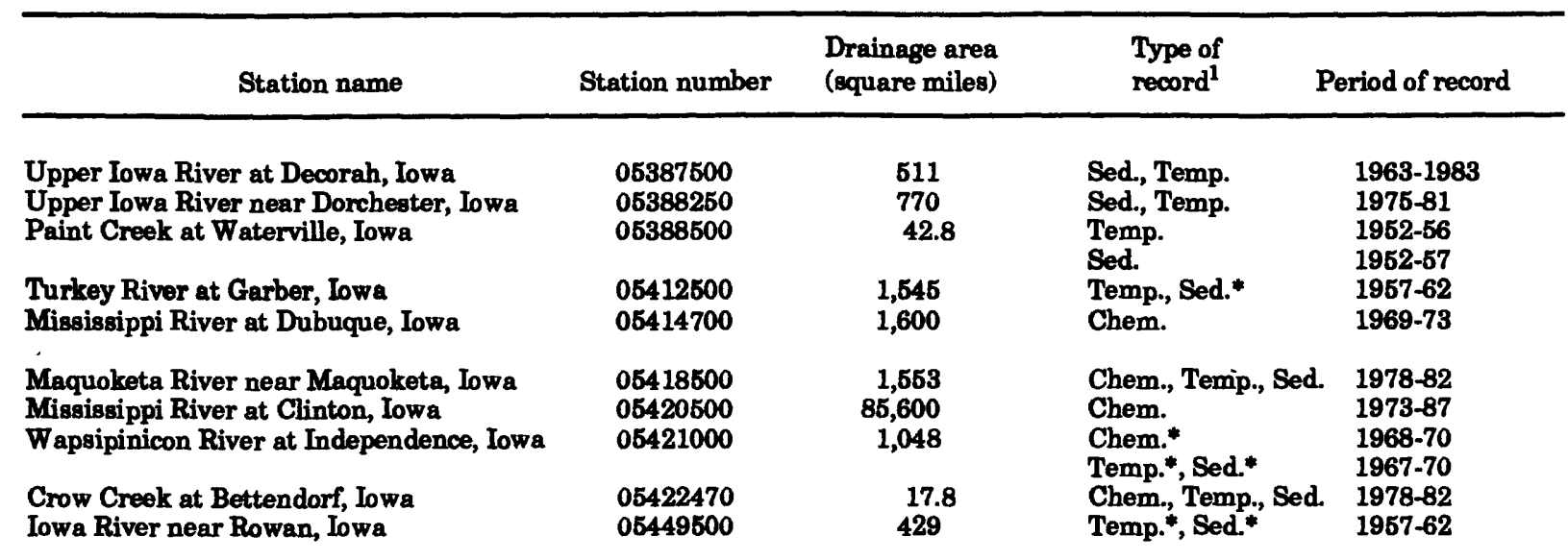


Table 4. Discontinued surface-water-quality stations in Iowa--Continued

\begin{tabular}{|c|c|c|c|c|}
\hline Station name & Station number & $\begin{array}{l}\text { Drainage area } \\
\text { (square miles) }\end{array}$ & $\begin{array}{l}\text { Type of } \\
\text { record }\end{array}$ & Period of record \\
\hline $\begin{array}{l}\text { Cedar River near Gilbertville, Iowa } \\
\text { Iowa River at Iowa City, Iowa } \\
\text { Ralston Creek at Iowa City, Iowa }\end{array}$ & $\begin{array}{l}05464020 \\
05454500 \\
05455000\end{array}$ & $\begin{array}{l}5,234 \\
3,271 \\
\quad 3.01\end{array}$ & $\begin{array}{l}\text { Chem. } \\
\text { Chem., Temp., Sed. } \\
\text { Chem., Temp., Sed. }\end{array}$ & \multirow{2}{*}{$\begin{array}{l}1971,1975-81 \\
1952-1987 \\
1906-1907 \\
1944-88 \\
1969-74 \\
1969-74\end{array}$} \\
\hline $\begin{array}{l}\text { Fourmile Creek near Lincoln, Iowa } \\
\text { Half Mile Creek near Gladbrook, Iowa }\end{array}$ & $\begin{array}{l}05464130 \\
05464133\end{array}$ & $\begin{array}{r}13.78 \\
1.33\end{array}$ & $\begin{array}{l}\text { Chem., Temp., Sed. } \\
\text { Chem., Temp., Sed. }\end{array}$ & \\
\hline $\begin{array}{l}\text { Fourmile Creek near Traer, Iowa } \\
\text { Cedar River near Palo, Iowa } \\
\text { Cedar River at Cedar Rapids, Iowa }\end{array}$ & $\begin{array}{l}05464137 \\
05464450 \\
05464500\end{array}$ & $\begin{array}{l}19.51 \\
6,380 \\
6,640\end{array}$ & $\begin{array}{l}\text { Chem., Temp., Sed. } \\
\text { Chem. } \\
\text { Chem.* } \\
\text { Temp.* } \\
\text { Sed. }\end{array}$ & $\begin{array}{l}1969-74 \\
1975-79 \\
1906-07,1944-54 \\
1944-54 \\
1943-54\end{array}$ \\
\hline $\begin{array}{l}\text { Cedar River near Bertram, Iowa } \\
\text { Mississippi River at Burlington, Iowa }\end{array}$ & $\begin{array}{l}05464760 \\
05469720\end{array}$ & $\begin{array}{l}6,955 \\
4,000\end{array}$ & $\begin{array}{l}\text { Chem. } \\
\text { Chem. }\end{array}$ & $\begin{array}{l}1975-81 \\
1969-73\end{array}$ \\
\hline $\begin{array}{l}\text { Mississippi River at Keokuk, Iowa } \\
\text { Des Moines River at Fort Dodge, Iowa } \\
\text { Des Moines River at Des Moines, Iowa }\end{array}$ & $\begin{array}{l}05474500 \\
05480500 \\
05482000\end{array}$ & $\begin{array}{r}119,000 \\
4,190 \\
6,245\end{array}$ & $\begin{array}{l}\text { Chem. } \\
\text { Chem. } \\
\text { Chem. } \\
\text { Temp., Sed. }\end{array}$ & $\begin{array}{l}1974-87 \\
1972-73 \\
1954-55 \\
1954-61\end{array}$ \\
\hline $\begin{array}{l}\text { E. Fork Hardin Creek near Churdan, Iowa } \\
\text { M. Fork Raccoon River near Bayard, Iowa }\end{array}$ & $\begin{array}{l}05483000 \\
05483450\end{array}$ & $\begin{array}{l}24.0 \\
375\end{array}$ & $\begin{array}{l}\text { Temp.*, Sed.* } \\
\text { Chem., Temp., Sed. }\end{array}$ & $\begin{array}{l}1952-57 \\
1979-85\end{array}$ \\
\hline $\begin{array}{l}\text { M. Fork Raccoon River at Panora, Iowa } \\
\text { Raccoon River at Des Moines, Iowa } \\
\text { Des Moines River below Raccoon River } \\
\text { at Des Moines, Iowa }\end{array}$ & $\begin{array}{l}05483600 \\
05485000 \\
05485500\end{array}$ & $\begin{array}{r}440 \\
3,590 \\
9,770\end{array}$ & $\begin{array}{l}\text { Chem., Temp., Sed. } \\
\text { Chem., Temp. } \\
\text { Chem.* } \\
\text { Temp.*, Sed. }\end{array}$ & $\begin{array}{l}1979-85 \\
1945-47 \\
1944-45 \\
1944-47\end{array}$ \\
\hline $\begin{array}{l}\text { Des Moines River below Des Moines, Iowa } \\
\text { Middle River near Indianola, Iowa }\end{array}$ & $\begin{array}{l}05485520 \\
05486490\end{array}$ & $\begin{array}{r}9,901 \\
503\end{array}$ & $\begin{array}{l}\text { Chem. } \\
\text { Temp.*, Sed. }\end{array}$ & $\begin{array}{l}1971,1975-81 \\
1962-67\end{array}$ \\
\hline White Breast Creek near Dallas, Iowa & 05487980 & 342 & $\begin{array}{l}\text { Chem. } \\
\text { Temp., Sed. }\end{array}$ & $\begin{array}{l}1968-73 \\
1967-73\end{array}$ \\
\hline $\begin{array}{l}\text { Big Sioux River at Sioux City, Iowa } \\
\text { Missouri River at Sioux City, Iowa }\end{array}$ & $\begin{array}{l}06485950 \\
06486000\end{array}$ & $\begin{array}{r}9,410 \\
314,600\end{array}$ & $\begin{array}{l}\text { Chein. } \\
\text { Chem. }\end{array}$ & $\begin{array}{l}1969-73 \\
1972-86\end{array}$ \\
\hline $\begin{array}{l}\text { Floyd River at James, Iowa } \\
\text { Floyd River at Sioux City, Iowa } \\
\text { Missouri River at Decatur, Nebr. } \\
\text { Little Sioux River at Correctionville, } \\
\text { Iowa }\end{array}$ & $\begin{array}{l}06600500 \\
06600520 \\
06601200 \\
06606600\end{array}$ & $\begin{array}{r}882 \\
921 \\
316,160 \\
2,500\end{array}$ & $\begin{array}{l}\text { Temp., Sed. } \\
\text { Chem. } \\
\text { Chem. } \\
\text { Chem.* } \\
\text { Temp.* } \\
\text { Sed. }\end{array}$ & $\begin{array}{l}1968-73 \\
1969-73 \\
1974-81 \\
1954-55 \\
1951-62 \\
1950-62\end{array}$ \\
\hline Little Sioux River near Kennebec, Iowa & 06606700 & 2,738 & $\begin{array}{l}\text { Temp. } \\
\text { Sed. }\end{array}$ & $\begin{array}{l}1950-55 \\
1950-57\end{array}$ \\
\hline $\begin{array}{l}\text { Little Sioux River at River Sioux, Iowa } \\
\text { Soldier River near Mondamin, Iowa } \\
\text { Steer Creek near Magnolia, Iowa } \\
\text { Thompson Creek near Woodbine, Iowa } \\
\text { Willow Creek near Logan, Iowa }\end{array}$ & $\begin{array}{l}06607513 \\
06608505 \\
06609200 \\
06609590 \\
06609600\end{array}$ & $\begin{array}{l}3,600 \\
440 \\
9.26 \\
6.97 \\
129\end{array}$ & $\begin{array}{l}\text { Chem. } \\
\text { Chem. } \\
\text { Temp., Sed. } \\
\text { Temp., Sed. } \\
\text { Chem., Temp. } \\
\text { Sed. }\end{array}$ & $\begin{array}{l}1969-73 \\
1970-73 \\
1963-69 \\
1963-69 \\
1972-75 \\
1971-75\end{array}$ \\
\hline $\begin{array}{l}\text { Missouri River at Omaha, Nebr. } \\
\text { Mule Creek near Malvern, Iowa }\end{array}$ & $\begin{array}{l}06610000 \\
06808000\end{array}$ & $\begin{array}{r}322,800 \\
10.6\end{array}$ & $\begin{array}{l}\text { Chem. } \\
\text { Temp. } \\
\text { Sed. }\end{array}$ & $\begin{array}{l}1969-86 \\
1958-69 \\
1954-69\end{array}$ \\
\hline $\begin{array}{l}\text { Davids Creek near Hamlin, Iowa } \\
\text { East Nishnabotna River at Red Oak, Iowa } \\
\text { Platte River near Diagonal, Iowa } \\
\text { Thompson River at Davis City, Iowa }\end{array}$ & $\begin{array}{l}06809000 \\
06809500 \\
06818750 \\
06898000\end{array}$ & $\begin{array}{l}26.0 \\
894 \\
217 \\
701\end{array}$ & $\begin{array}{l}\text { Temp.* } \\
\text { Temp., Sed. } \\
\text { Chem. } \\
\text { Chem. } \\
\text { Temp., Sed. }\end{array}$ & $\begin{array}{l}1952-53,1965-68 \\
1962-73 \\
1969-73 \\
1967-73 \\
1968-73\end{array}$ \\
\hline Weldon River near Leon, Iowa & 06898400 & 104 & Chem. & $1968-73$ \\
\hline $\begin{array}{l}\text { Chariton River near Chariton, Iowa } \\
\text { Honey Creek near Russell, Iowa. } \\
\text { Chariton River near Rathbun; Iowa }\end{array}$ & $\begin{array}{l}06903400 \\
06903500 \\
06903900\end{array}$ & $\begin{array}{l}182 \\
13.2 \\
551\end{array}$ & $\begin{array}{l}\text { Temp., Sed. } \\
\text { Sed. } \\
\text { Temp*, Sed.* }\end{array}$ & $\begin{array}{l}1969-73 \\
1952-62 \\
1962-69\end{array}$ \\
\hline
\end{tabular}

\footnotetext{
1 Type of record: Chem., chemical quality; Temp., water temperature; Sed., sediment.
} 
SEDIMENT STATIONS

(IA 00-004)

PERIOD OF PROJECT: Continuous since 1943

PROJECT CHIEF: D.J. O’Connell

STUDY AREA: Statewide

COOPERATING AGENCIES: U.S. Geological

Survey (Federal program), U.S. Army

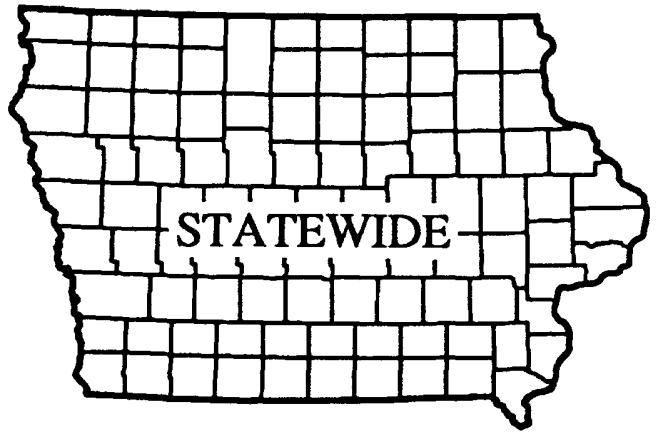

Corps of Engineers, Iowa Department of Natural Resources (Geological

Survey Bureau), and the University of Iowa (Department of Preventive Medicine).

NEED FOR STUDY: Water-resource planning and water-quality assessment requires a nationwide base of standardized information. Sediment concentrations and discharges in rivers and streams need to be monitored and defined to achieve this goal.

OBJECTIVES: To provide a national sediment data base for use in Federal programs and Federal management of interstate waters. The State also needs spatial and temporal averages and trends of sediment concentration, sediment discharge, and particle size of sediment being transported in the rivers and streams for planning and action programs.

PROGRESS: Data were collected, compiled, and processed for publication on schedule. The annual data report, "Water Resources Data--Iowa, Water Year 1990" was delivered in May 1991. The network consist of five daily sediment stations operated in cooperation with the Iowa Department of Natural Resources (Geological Survey Bureau) [IDNR (GSB)]. Two daily stations were operated in cooperation with the U.S. Army Corps of Engineers, Rock Island District, and one daily sediment station with the U.S. Army Corps of Engineers, St. Paul District. A program operated cooperatively with the U.S. Army Corps of Engineers, Omaha District, studied the larger than 0.062-millimeter material in the river cross section and the point velocities at three sites on the Missouri River. A daily sediment station was added to the network during 1991 that is operated cooperatively with the University of Iowa (Department of Preventive Medicine) and the Agricultural Research Service. Two daily sediment stations were added during 1992 in cooperation with the IDNR (GSB) to study the effect of land-use practices on the stream's sediment yield.

Laboratory analyses of sediment samples collected by the U.S. Army Corps of Engineers (Rock Island District) and by U.S. Geological Survey Districts in Alaska, Arizona, Colorado, Illinois, Indiana, Kentucky, Minnesota, Missouri, Montana, Nebraska, Nevada, North Dakota, South Dakota, Tennessee, Wisconsin, and Wyoming are performed in the Iowa District sediment laboratory. The laboratory performs the following analyses for suspended sediment: (1) concentration; (2) concentration and determination of material finer than sand; and (3) dissolved solids. Particle-size analyses for suspended sediment, bed material, and bed-load material that are sand are determined by sieve or visual accumulation tube, and particle size of material finer than sand by pipet, Sedigraph, or air elutriation. 
IOWA PRECIPITATION MONITORING

FOR THE NATIONAL TRENDS

NETWORK

(IA 84-005)

PERIOD OF PROJECT: Continuous since 1984

PROJECT CHIEF: R.B. Lambert

STUDY AREA: Clayton and Lucas Counties

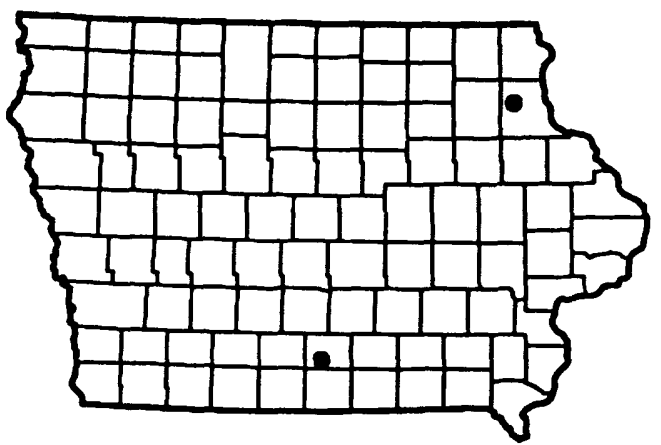

COOPERATING AGENCY: U.S. Geological Survey (Federal program).

NEED FOR STUDY: The amount of substances dispersed into the atmosphere and deposited by precipitation, aerosols, and gases is expected to continue to increase throughout North America. Thus, there is an increasing need for careful, coordinated measurement on a national scale of the amounts, nature, and effects of these substances. To establish long-term trends in composition and flux, it is necessary that these measurements be carried out for a period of at least 10 years. These measurements will be used to assess the link between environmental effects and atmospheric deposition and will provide the information necessary for the responsible management of the agricultural, forest, and aquatic ecosystems of the United States.

OBJECTIVES: The Iowa precipitation-monitoring stations are part of the National Atmospheric Deposition Program/National Trends Network (NADP/NTN) program to provide a regional-to-national overview of chemical composition of atmospheric deposition in the United States. This program includes scientific research in atmospheric deposition, monitoring, and assessment activities. Information from this overview is being used to discover and characterize environmentally important geographical and temporal trends in the chemical climate of North America. In addition, information from this national program is being used to assess the effects of atmospheric deposition on: (1) the productivity of agricultural and forest lands; (2) the health of domestic animals, wildlife, and fish; (3) the chemistry of surface and ground water; and (4) visibility and materials.

PROGRESS: Precipitation samples are collected at 200 sites across the United States. Wet-deposition samples are collected weekly on a continuing basis at the Big Spring Fish Hatchery in Clayton County, and at the McNay Agricultural Research Farm in Lucas County (see index map). Severe flooding in the Turkey River basin in northeast lowa destroyed the Big Spring Fish Hatchery sampler in August 1990 and again in June 1991. After the June 1991 flood, equipment at that site was again replaced, and the station returned to operation in July 1991. Information collected from the Iowa stations is published annually in a series of data reports. Values of $\mathrm{pH}$ and specific conductance and the chemical composition of the precipitation from both stations were published in the "Water Resources Data--Iowa, Water Year 1990" and the U.S. Geological Survey's "NADP/NTN Annual Data Summary-Precipitation Chemistry in the United States for 1989." The results of sample analyses for water year 1991 will be published in "Water Resources Data--Iowa, Water Year 1991." 
WATER USE

(IA 00-007)

PERIOD OF PROJECT: Continuous since 1980

PROJECT CHIEF: E.E. Fischer

STUDY AREA: Statewide

COOPERATING AGENCY: Iowa Department of Natural Resources

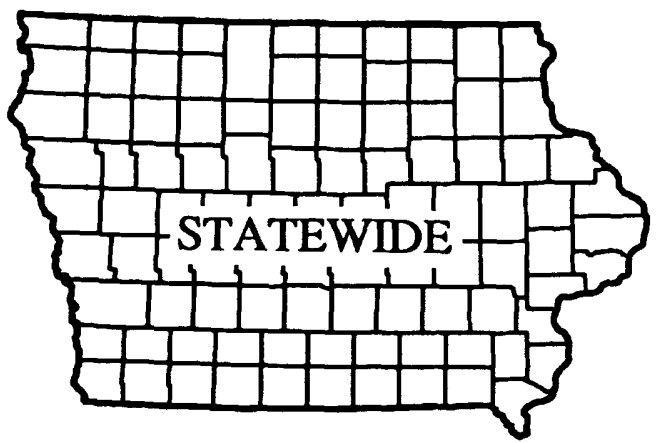

NEED FOR STUDY: In 1977, the Congress of the United States directed the U.S. Geological Survey to establish a National Water-Use Information Program to complement the Survey's data on the Nation's water resources. This program, which became a part of the U.S. Geological Survey's Federal-State cooperative program, was designed to be the source for accurate, consistent, timely, and accessible water-use information.

OBJECTIVES: The objective of the Iowa water-use program is to collect and compile reliable site-specific and aggregated water-use information for the State in accordance with guidelines established for the National Water-Use Information Program. Data are to be collected annually and the data base updated to maintain current information on the State's water use.

PROGRESS: The Iowa part of the 1990 national water-use data-collection effort was completed during fiscal year 1991. Much of the data were supplied by water-user reports to the Iowa Department of Natural Resources. The remainder of the data were collected by contacting individual water users (municipal, commercial, and industrial) and by using documented estimation procedures. The data were sent to the National Water-Use Information Program office. The data that were collected include the amount of water each user withdrew during 1990, whether the withdrawal was ground water or surface water, and the location of each withdrawal point. Estimates of water use on farms (other than irrigation, which is covered by the State's water-permit system) were made from data supplied by the Iowa Department of Agriculture and Land Stewardship. Estimates of domestic water use were provided by the billing departments of municipalities representing more than one-half of the State's population. 


\section{AREAL HYDROLOGIC INVESTIGATIONS}


FLOOD INFORMATION AT SELECTED BRIDGE AND CULVERT SITES

\section{(IA 66-006)}

PERIOD OF PROJECT: Continuous since 1966

PROJECT CHIEF: D.A. Eash

STUDY AREA: Statewide

COOPERATING AGENCY: Iowa Department of

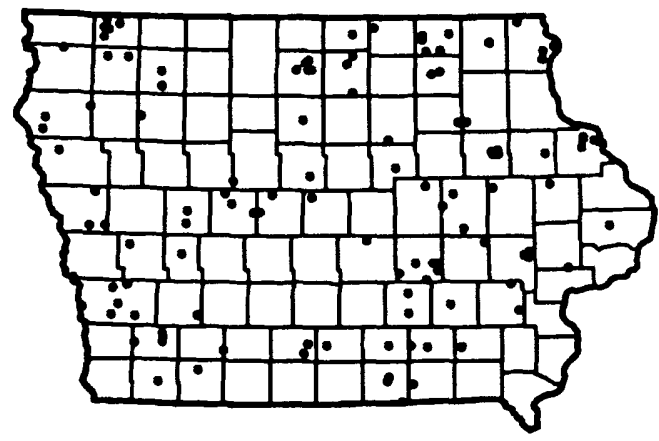

Transportation (Highway Division, Highway Research Advisory

Board).

NEED FOR STUDY: Systematic flood information is needed for the proper hydraulic design of new bridges, culverts, and other flow structures (especially in small drainage basins of less than $100 \mathrm{mi}^{2}$ ) and for the evaluation of existing structures. There also is a need to analyze the hydrology and hydraulics of proposed sites with little available data and to document outstanding floods at ungaged sites on an event basis. Because of the large number of small basins in the State, relatively few will ever have specific flood data available. Therefore, flood discharges are estimated from numerical models that are calibrated using data collected for these sites. To define and calibrate these models for basins in Iowa, flood data must be available from basins with a variety of characteristics, such as drainage area, topography, soil type, shape, and land use.

OBJECTIVES: For small drainage basins with less than $100 \mathrm{mi}^{2}$ of drainage area: (1) obtain basin characteristics and systematic flood data for a network of representative basins in the State, and (2) document peak discharges on ungaged basins on an event basis.

PROGRESS: Operation of the small-basin, crest-stage gage (CSG) network was continued. Annual peak-flood data from the network were compiled and published in the U.S. Geological Survey Water-Data Report IA-90-1 (O'Connell and others, 1990). Data were collected from 120 CSG sites throughout the State (see index map). During water year 1990, 22 CSG sites were discontinued, and 17 new sites were added to the network. Maximum gage heights were recorded at the 17 sites with peak discharges determined at 7 of the sites. Maximum gage heights were recorded, and peak discharges were determined at 46 sites. Maximum gage heights were recorded at 18 sites, and peak discharges will be published when ratings for the sites are defined. At 11 sites, a peak above the base did not occur. Two sites were affected by ice during the time of the maximum gage height, and no discharge could be determined. Three indirect discharge computations and field work for five indirect discharge measurements and culvert ratings were completed in 1990. Six indirect discharge measurements started in water year 1990 were finalized and submitted for review in water year 1991. Field work for a bridge study on the Cedar River at Cedar Falls was completed. Site selection and installation of new CSG sites continued. 


\section{FLOOD PROFILES OF IOWA STREAMS (IA 58-011)}

PERIOD OF PROJECT: Continuous since 1957

PROJECT CHIEF: P.J. Soenksen

STUDY AREA: Statewide

COOPERATING AGENCY: Iowa Department of Transportation (Highway Division, Highway Research Advisory Board).

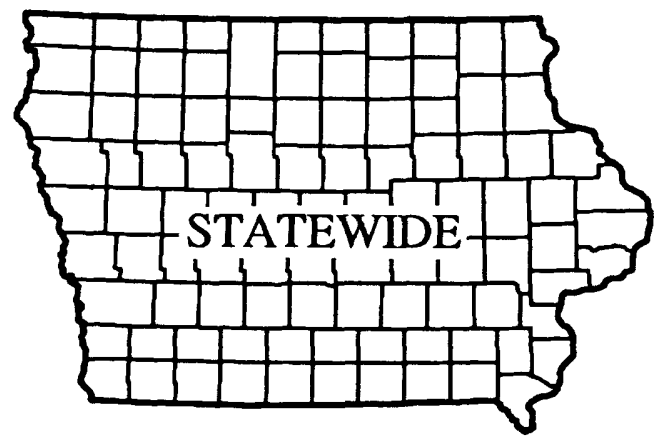

NEED FOR STUDY: Information is needed on flood peaks and profiles for the economical and safe location and design of bridges and other structures on or over streams and the adjacent flood plains. Defining the limits of flood inundation and establishing encroachment limits on flood plains are related problems needing this information. Basic data on major floods are needed to compute flood-peak discharges and calibrate water-surface profile models at ungaged sites along streams.

OBJECTIVES: Define the water-surface profiles and corresponding discharges along streams in basins with at least $100 \mathrm{mi}^{2}$ of drainage area for at least one recorded flood and the expected flood(s) of one or more selected recurrence intervals, usually the 25- and 50-year recurrence intervals. Evaluate the flood characteristics and hydraulics at existing and proposed flow structures on basins of all sizes on a request basis.

PROGRESS: Low-water and low-bridge chord and bridge-deck elevations were determined using temporary bench marks along the East and West Nishnabotna Rivers. The Nishnabotna report was published as U.S. Geological Survey Open-File Report 91-171, "Floods in the Nishnabotna River Basin, Iowa" (Eash and Heintz, 1991). As a result of significant floods, high-water marks, low-bridge chord elevations, and bridge-deck elevations were surveyed using temporary bench marks along Clear Creek in Johnson County (flood of June 17, 1990), Perry Creek in Woodbury County (flood of May 19, 1990), along Squaw Creek and the Skunk River near and in Ames in Story County (flood of June 17, 1990), and in the Turkey River basin in northeast Iowa (flood of June 6, 1991). Level lines were measured using sea level datum from bench marks to establish temporary marks on bridges along Willow Creek, Mosquito Creek, and the South Raccoon River for the Raccoon River report using third-order accuracy. The report for the Raccoon River basin, "Floods of 1986 and 1990 in the Raccoon River Basin, Iowa" is in preparation. Elevations of high-water marks were obtained at three bridge sites in the Waterloo-Cedar Falls area in Black Hawk County for site-specific documentation of backwater and flood conditions for the flood of July 30,1990, along the Cedar River. The following is a chronological listing of flood-profile reports for Iowa prepared by the U.S. Geological Survey. 


\section{CHRONOLOGICAL FLOOD-PROFILE \\ REFERENCES}

Schwob, H.H., 1963, Cedar River basin floods: Iowa Highway Research Board Bulletin 27, Iowa Department of Transportation, Ames, $59 \mathrm{p}$.

Schwob, H.H., and Meyers, R.E., 1965, The 1965 Mississippi River flood in Iowa: Iowa City, U.S. Geological Survey Open-File Report, 39 p.

Schwob, H.H., 1966, Little Sioux River basin floods: Iowa City, U.S. Geological Survey Open-File Report, 60 p.

1967, Floods on Otter Creek in Linn County, Iowa: Iowa City, U.S. Geological Survey Open-File Report, 22 p.

1968, Flood of June 7, 1967, in the Wapsinonoc Creek basin, Iowa: Iowa City, U.S. Geological Survey Open-File Report, 21 p.

1968, Flood profile study, Squaw Creek, Linn County, Iowa: Iowa City, U.S. Geological Survey Open-File Report, 13 p.

1970a, Floods in the upper Des Moines River basin, Iowa: Iowa City, U.S. Geological Survey Open-File Report, 49 p.

1970b, Flood profile study, Morgan Creek, Linn County, Iowa: Iowa City, U.S. Geological Survey Open-File Report, 16 p.

1970c, Flood of March 3, 1970, on Old Mans Creek, Johnson County, Iowa: Iowa City, U.S. Geological Survey Open-File Report, 9 p.

1970d, Flood profile study, Hoosier Creek, Linn County, Iowa: Iowa City, U.S. Geological Survey Open-File Report, 18 p.

1971, Floods in the Wapsipinicon River basin, Iowa: Iowa City, U.S. Geological Survey Open-File Report, 52 p.

Heinitz, A.J., 1973a, Floods in the Iowa River basin upstream from Coralville Lake, Iowa: Iowa City, U.S. Geological Survey Open-File Report, 75 p.

1973b, Flood of August 2, 1972, in the Little Maquoketa River basin, Dubuque County, Iowa: Iowa City, U.S. Geological Survey Open-File Report, 28 p.

1973c, Floods in the Rock River basin, Iowa: Iowa City, U.S. Geological Survey Open-File Report, 74 p.

Lara, O.G., and Heinitz, A.J., 1976, Flood of June 27, 1975, in city of Ames, Iowa: U.S. Geological Survey Open-File Report 76-728, $56 \mathrm{p}$.

Heinitz, A.J., 1977, Floods in the Big Creek basin, Linn County, Iowa: 
U.S. Geological Survey Open-File Report 77-209, 35 p.

Heinitz, A.J., and Wiitala, S.W., 1978, Floods in the Skunk River basin, Iowa: U.S. Geological Survey Open-File Report 79-272, $80 \mathrm{p}$.

Heinitz, A.J., 1979, Supplement to floods in the upper Des Moines River basin, Iowa: U.S. Geological Survey Open-File Report 79-1486, 6 p.

1980, Floods in the Raccoon River basin, Iowa: U.S. Geological Survey Open-File Report 80-162, 110 p.

Heinitz, A.J., and Riddle, D.E., 1981, Floods in the English River basin, Iowa: U.S. Geological Survey Open-File Report 81-67, $61 \mathrm{p}$.

Heinitz, A.J., 1985, Floods in south-central Iowa: U.S. Geological Survey Open-File Report 85-100, 95 p.

1986, Floods in the Floyd River basin, Iowa: U.S. Geological Survey Open-File Report 86-476, 61 p.

Eash, D.A., and Heinitz, A.J., 1991, Floods in the Nishnabotna River basin, Iowa: U.S. Geological Survey Open-File Report 91-171, $118 \mathrm{p}$. 
IOWA GROUND-WATER-QUALITY

MONITORING PROGRAM

(IA 83-047)

PERIOD OF PROJECT: Continuous since 1982

PROJECT CHIEF: D.A. Sneck-Fahrer

STUDY AREA: Statewide

COOPERATING AGENCIES: Iowa Department of Natural Resources (Geological Survey Bureau) and the University of Iowa Hygenic Laboratory.

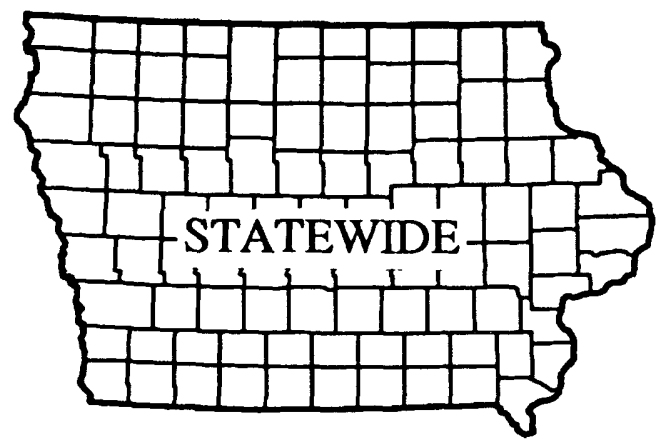

NEED FOR STUDY: Ground-water quality is one of the most critical resource concerns in the State of Iowa. A ground-water-quality monitoring program is needed to provide data to aid State and local management and regulatory agencies to effectively evaluate the ground-water resources of the State.

OBJECTIVES: The primary purpose of the ground-water-quality monitoring program is to provide consistent and representative ground-water-quality data that describe the chemical quality of the ground-water resources in the State. The two major objectives of this program are: (1) to specifically describe the baseline ground-water quality of the major aquifers in the State and to provide ground-water-quality data for areas of the State or aquifers in the State that are stressed by intensive use, contamination, or deteriorating quality; and (2) to establish a subnetwork within the program to monitor long-term ground-water-quality trends.

PROGRESS: Approximately 2,000 municipal wells are included in the ground-water-quality monitoring inventory of potential sampling sites, 200 of which are selected annually. The location of sampling sites for 1990 and 1991 is shown in figure 7. The current sampling strategy, which was initiated in 1990 , combines a random selection from the inventory of wells, weighted by aquifer vulnerability, with a fixed subnetwork of 50 wells. This selection process provides a more statistically sound basis for the assessment of the overall quality of ground water throughout the State and for the analysis of long-term water-quality trends.

During 1991 sampling, personal contact with municipal-well superintendents resulted in updating and confirmation of $\mathbf{4 0}$ percent of the ground-water-quality monitoring inventory. Additionally, land use within a 2-mi radius surrounding each well was assessed. All samples were collected according to standard U.S. Geological Survey 
procedures and chemically analyzed by the University of Iowa Hygenic Laboratory for constituents in the following categories: (1) major ions, (2) nutrients, (3) common herbicides, (4) selected priórity contaminants, and (5) radiochemical materials. Analytical and land-use data from 1991 currently are being compiled and will be used in conjunction with data from previous years for subsequent statistical analysis to explore the spatial and temporal aspects of water quality in Iowa.

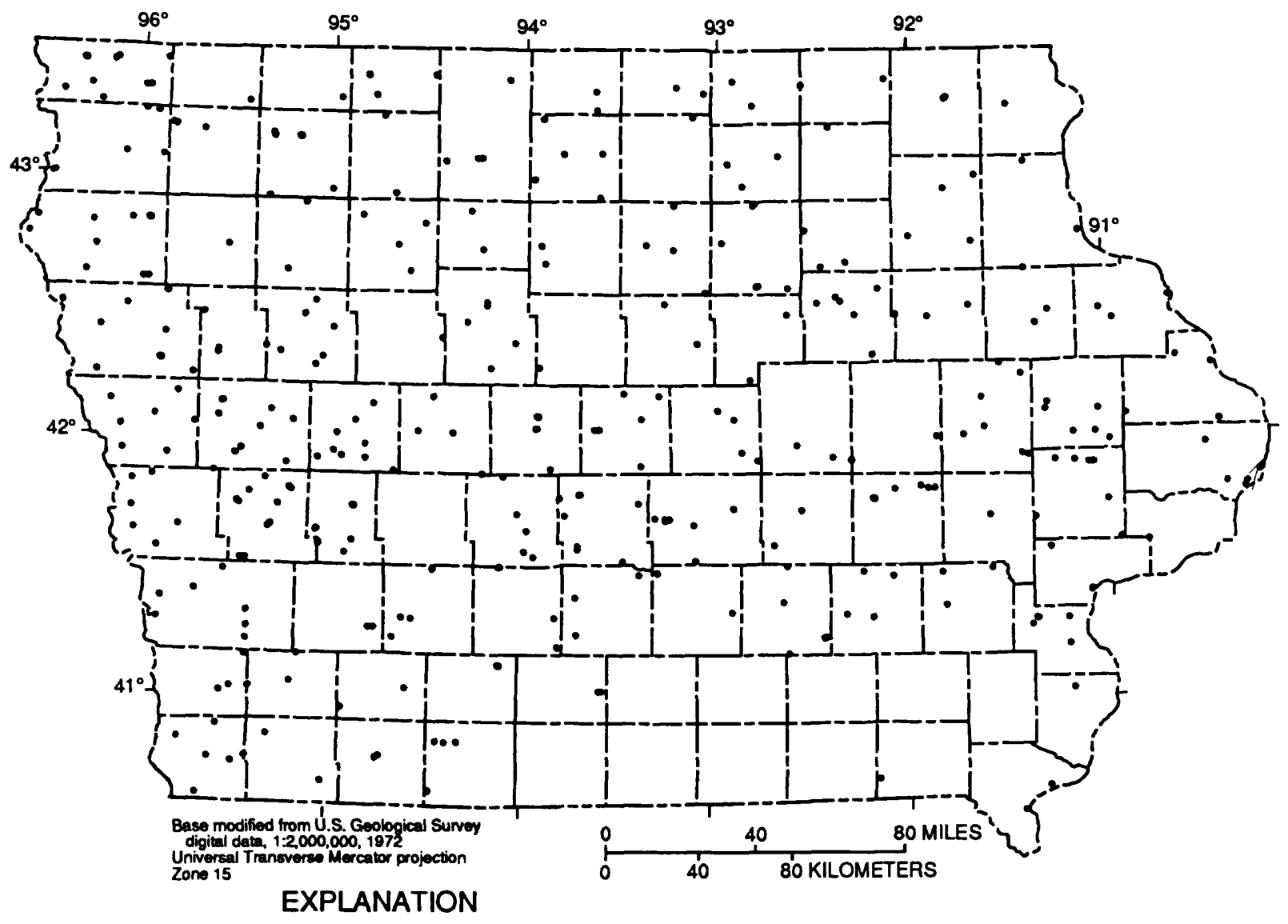

- GROUND-WATER-QUALITY SAMPLING SITE

Figure 7. Location of ground-water-quality sampling sites. 
DES MOINES AIR NATIONAL GUARD BASE, INSTALLATION RESTORATION PROGRAM

(IA 84-050)

PERIOD OF PROJECT: 1984-92

PROJECT CHIEF: R.C. Buchmiller

STUDY AREA: Polk County

COOPERATING AGENCY: U.S. Department of Defense (Air Force Center for Environmental Excellence).

NEED FOR STUDY: Disposal of chemicals at sites on the Iowa Air National Guard Base, Des Moines, may pose a hazard to ground and surface water in the study area.

OBJECTIVES: To identify and evaluate potential problems caused by past handling or disposal of toxic or hazardous materials at the U.S. Air Force facility.

PROGRESS: Four sites where disposal of wastes or leakage of stored materials occurred were studied. A total of 24 observation wells were drilled and completed at the four sites. Water levels were measured in the wells to obtain information on ground-water flow direction and gradient at each site. Hydraulic tests were performed on each well to characterize the hydraulic properties of soils at each site. Twelve wells were sampled for selected water-quality constituents and properties during 1984 to determine if disposal of wastes or leakage of stored materials had affected ground water adjacent to the sites. On the basis of results from this sampling, an additional 12 wells were installed, and all 24 wells were sampled twice during 1987 to confirm the presence of selected water-quality constituents and properties. In addition to the sampling of the observation wells, a soil-gas survey was performed during 1987 to attempt to map the areal extent of suspected ground-water contamination plumes. Onsite work has been completed, and the data have been presented to the U.S. Air Force for their approval. Plans for fiscal year 1992 include publishing the final report and the proper abandonment of monitoring wells at the sites selected for no further action. 
SOUTHWEST IOWA GROUND-WATER APPRAISAL (IA 85-053)

PERIOD OF PROJECT: 1985-90

PROJECT CHIEF: R.C. Buchmiller

STUDY AREA: Adair, Adams, Cass, Fremont, Mills, Montgomery, Page, Pottawattamie, and Taylor Counties

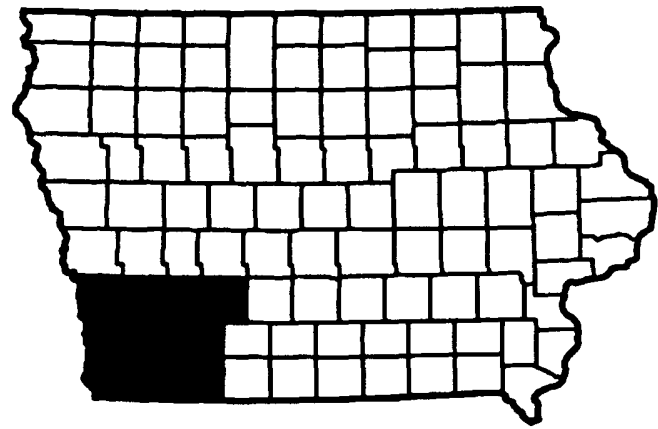

COOPERATING AGENCY: Iowa Department of Natural Resources (Geological Survey Bureau).

NEED FOR STUDY: Southwest Iowa is an agricultural area with limited sources of ground water. Possible ground-water sources with adequate quality and quantity of water are the alluvial, buried channel, and Dakota aquifers. Other aquifers exist but are of a discontinuous nature or contain water of undesirable quality.

OBJECTIVES: To determine the availability, quantity, and quality of ground water from the alluvial, glacial-drift, and Dakota aquifers in southwest Iowa. Specific objectives are to: (1) determine the location, areal extent, and use of these aquifers; (2) evaluate the occurrence, movement, and storage of ground water, including the relation between streams and near-surface aquifers; (3) estimate the potential yields to wells tapping the aquifers; (4) describe the chemical quality of the surface and ground water; and (5) report the annual municipal water withdrawals and estimate other categories of water use from the aquifers.

PROGRESS: A study of the shallow ground-water resources in a nine-county area of southwest Iowa was begun in 1985 . Historical records and test-well drilling were used to obtain data for specific aquifers in areas where little or no ground-water information was available. Observation wells were constructed to monitor water-level changes and to obtain samples of ground water for chemical analysis. Surface-water samples were collected at several sites to compare with analytical results from adjacent alluvial aquifers. Municipal water-use information was obtained from local water suppliers. Data were collected for five types of unconsolidated aquifers and one bedrock aquifer; alluvial, loess, inter-till sand-and-gravel, basal sand-and-gravel, buried-channel, and the Dakota aquifers, respectively. Data collection for this project has been completed. A report authored by personnel of the U.S. Geological Survey and Iowa Department of Natural Resources (Geological Survey Bureau) was published (Hansen and others, 1992). An additional report that revises the bedrock topography of the area on the basis of data compiled during this project is in preparation. 
PERIOD OF PROJECT: 1985-88

PROJECT CHIEF: S.J. Kalkhoff

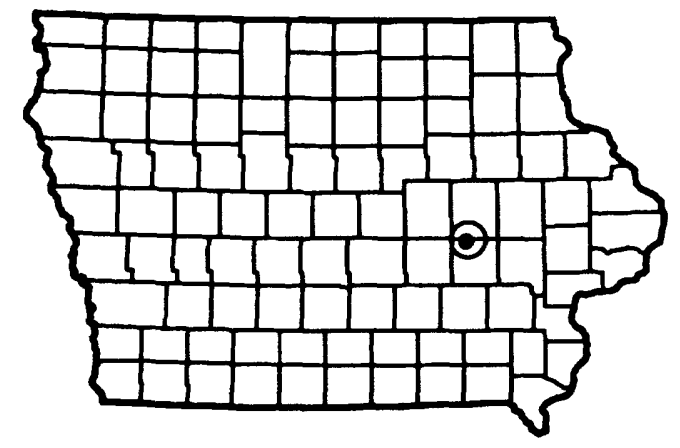

STUDY AREA: Iowa County

COOPERATING AGENCY: The University of Iowa Hygienic Laboratory.

NEED FOR STUDY: Ground-water-quality monitoring programs have detected pesticides, particularly the herbicides alachlor, atrazine, and cyanazine, in a substantial number of wells in shallow ground-water supplies throughout Iowa. Increasing concern about the undesirable leaching of agricultural chemicals from fields to underlying aquifers has led to a need for detailed study of the movement of these chemicals.

OBJECTIVES: The purpose of this study is to evaluate the movement and distribution of selected pesticides in the field. The investigation focuses on the leaching of herbicides and nitrate to shallow ground water. Specific objectives are to determine the distribution of selected herbicides in the soil-unsaturated zone-aquifer profile and to interpret the movement, leaching rates, and physical-chemical characteristics of herbicide movement from the soil to shallow ground water.

PROGRESS: Seven wells and four lysimeters were completed at various depths at a site in the Iowa River alluvial aquifer near Marengo, Iowa. Water levels were measured in each well to determine the vertical gradient of water movement at the site. The wells and lysimeters were sampled monthly for 2 years. Sampling was completed in the fall of 1987. Water samples were analyzed for nitrate and selected herbicides. Results and interpretations of data collected during the study are being prepared for publication in a technical journal. 
SIDE-LOOKING AIRBORNE RADAR AS

A RECONNAISSANCE TOOL FOR THE DELINEATION OF LINEAMENTS IN WESTERN IOWA

(IA 86-056)

PERIOD OF PROJECT: 1986-92

PROJECT CHIEF: J.P. Caldwell

STUDY AREA: Adams, Cass, Crawford, Montgomery,

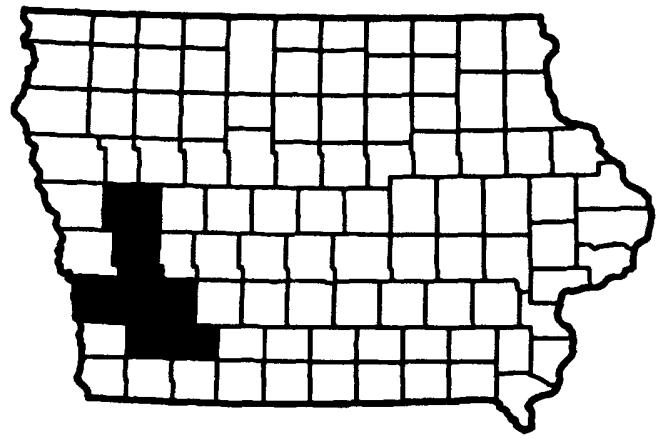

Pottawattamie, and Shelby Counties

COOPERATING AGENCY: U.S. Geological Survey (Federal program).

NEED FOR STUDY: The character and distribution of surface lithologic units have a major effect on the movement of surface and shallow ground water. Much of the ground-water availability or flow from carbonate bedrock in lowa is related to secondary permeability associated with structural features such as fractures, joints, and faults. The surface expression of bedrock structural features that have been mantled by glacial drift often is undetectable using routine field-reconnaissance methods. Mapping these bedrock features would aid in the protection, conservation, and utilization of these shallow aquifers. Side-looking airborne-radar (SLAR) imagery may provide a means of detecting fracture zones (lineaments) on a regional scale.

OBJECTTVES: Evaluate the usefulness of SLAR imagery for hydrologic and geologic reconnaissance in Iowa. SLAR imagery will be analyzed to detect subtle topographic expression, differences in texture and composition of surficial materials and vegetation, and drainage patterns that may follow the surface projection of bedrock-fracture zones. Regional maps showing the distribution of interpreted lineament systems will be evaluated according to known land-use patterns, cultural features, geology, and hydrology to select sites for detailed characterization studies. Bedrock-fracture zones may provide pathways for the migration of bedrock-sourced radiochemicals (radon). Streams will be sampled during base-flow conditions in the vicinity of these lineaments to determine if increased levels of radon are present.

PROGRESS: Preliminary maps showing the distribution of lineament systems in the Omaha quadrangle $\left(1^{\circ}\right.$ by $\left.2^{\circ}\right)$ were prepared utilizing SLAR imagery. Seven sites were selected for ground-water radon sampling on the basis of their close proximity to lineament intersections. Three of the sites were abandoned after onsite investigation showed them to be either inaccessible or that their streambed sediments were too fine grained to allow ground-water sampling with available field equipment. A total of 12 ground-waier samples were obtained from the four remaining sites. One sample at each site was collected where the stream crossed the mapped lineament intersection. Samples also were collected upstream and downstream from the primary sampling site to obtain background radon levels. The ground-water samples were analyzed onsite using a portable alpha scintillometer. Preliminary results show increased radon levels at three of the sites. 
EVALUATION OF FACTORS

AFFECTING THE OCCURRENCE OF

AGRICULTURAL CHEMICALS IN

WATER RESOURCES IN THE CENTRAL

MIDWEST

(IA 87-057)

PERIOD OF PROJECT: 1987-93

PROJECT CHIEF: M.R. Burkart

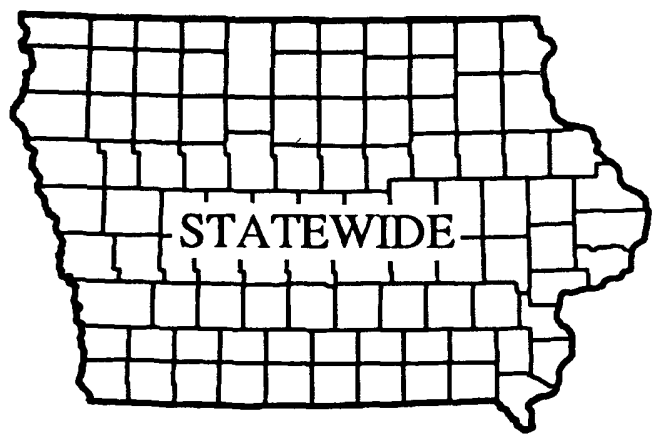

STUDY AREA: Ilinois, Indiana, and Iowa; parts of Kansas, Michigan, Minnesota, Missouri, Nebraska, North Dakota, Ohio, South Dakota, and Wisconsin

COOPERATING AGENCY: U.S. Geological Survey (Federal program).

NEED FOR STUDY: Agricultural chemical contamination of ground water and also of surface water poses a major threat in much of the United States. A comprehensive evaluation of the available information is needed to identify the extent to which water resources have been affected by agricultural chemicals and what measures are necessary to mitigate the problems related to contamination.

OBJECTIVES: In collaboration with the U.S. Department of Agriculture and the U.S. Environmental Protection Agency (EPA), the ultimate goal of this project is to develop an understanding of factors that affect the occurrence of agricultural chemicals in water resources and to develop strategies to improve and protect water resources. To meet this goal, two objectives being pursued by the U.S. Geological Survey are being coordinated within this project: (1) identify the spatial and temporal occurrence of herbicides and nitrate in water resources of the Midwest, and (2) evaluate the effect on water quality of prevailing and modified farming systems. The first objective is being approached through reconnaissance sampling and evaluation of chemical constituents of aquifers, streams, and precipitation throughout the 12-state area (fig. 8). Stream and atmospheric sampling is being coordinated through another U.S. Geological Survey project in Denver, Colorado. The project described here is conducting the ground-water reconnaissance and providing geographic-information-system support for the stream-sampling activity. The second objective is being met through collaborative efforts at five Management System Evaluation Areas (MSEA) in Iowa, Minnesota, Missouri, Nebraska, and Ohio. Water-quality research is being conducted through U.S. Geological Survey projects in the five states, and this research is being coordinated through this project.

PROGRESS: Progress on the first objective includes drafting and publishing a work plan for the ground-water reconnaissance. Samples were collected at 300 wells in shallow unconsolidated and bedrock aquifers in late winter 1991 and during July-August 1991. Among the reports in progress are the following topics: "Herbicides in Surface Waters of the Midwestern United States," "Effects of the Spring Flush," "Historical Occurrence of 
Nitrate in Ground Water of the MidContinent," and "Occurrence of Herbicides and Nitrate in Shallow Aquifers of the MidContinent." Several reports describing the MSEA plans and preliminary interpretation of regional data have been published. MSEA activities are fully in place. The EPA plans to initiate agro-ecosystem research at one or more MSEA sites in fiscal year 1992. A strategy is being developed to project the potential benefits of implementing MSEA research results to large segments of the MidContinent.

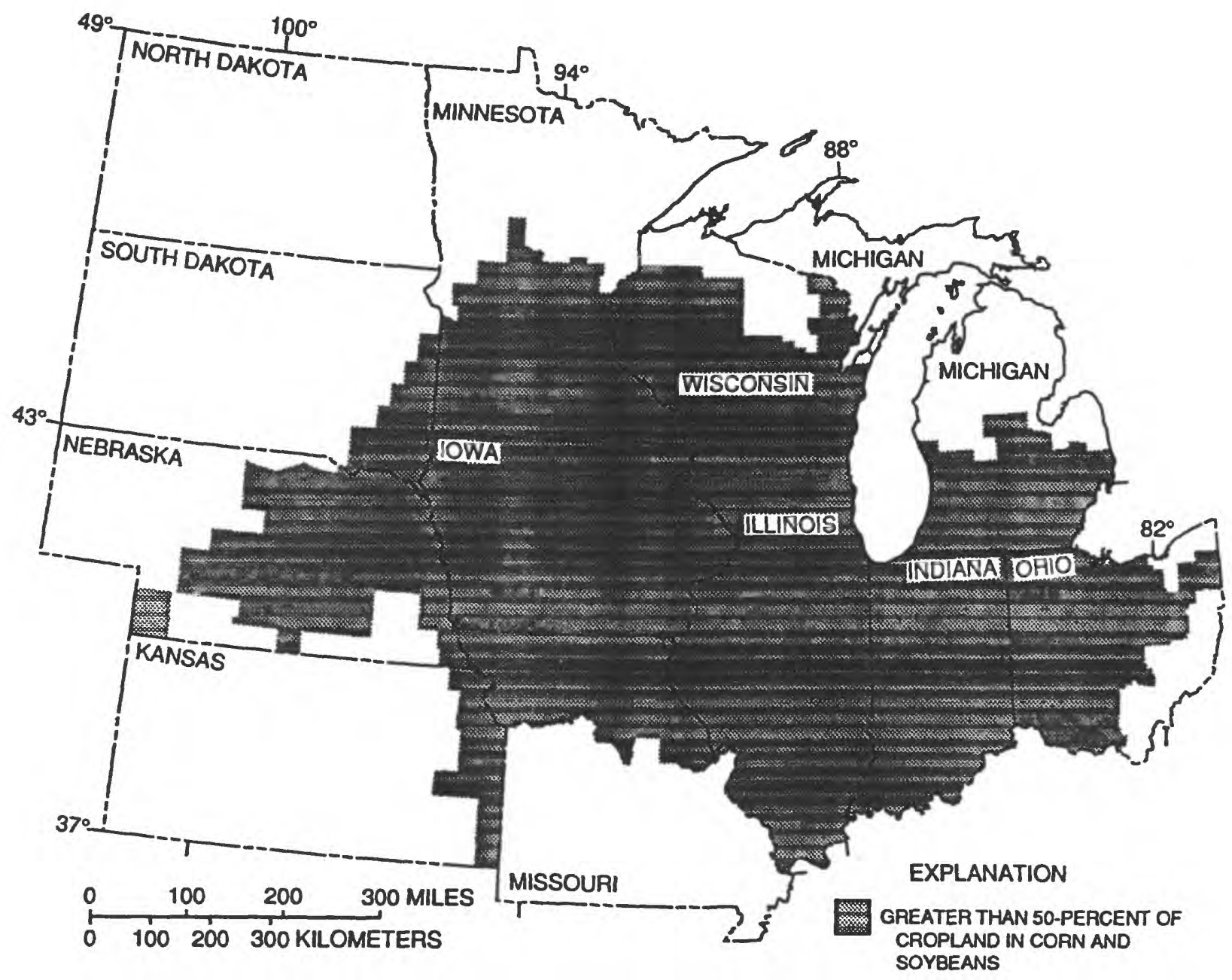

Figure 8. Area of focus for interagency research into the occurrence of agricultural chemicals in the water resources of the central Midwest. 
HYDROLOGIC ANALYSIS OF WATER QUALITY AND THE FLOW SYSTEM IN THE BIG SPRING BASIN, CLAYTON COUNTY, IOWA

(LA 87-058)

PERIOD OF PROJECT: 1987-92

PROJECT CHIEF: S.J. Kalkhoff

STUDY AREA: Clayton County

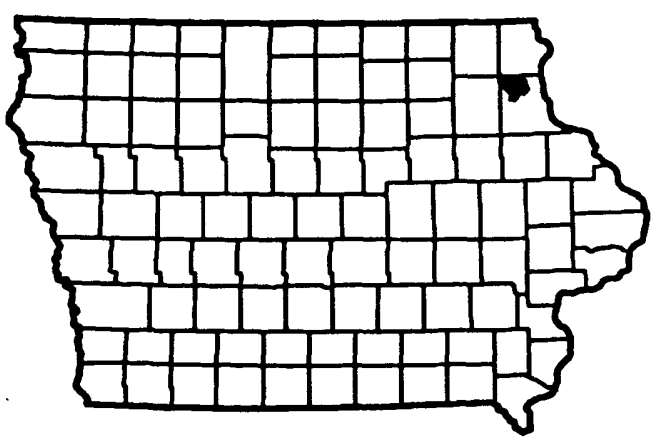

COOPERATING AGENCY: Iowa Department of Natural Resources (Geological Survey Bureau).

NEED FOR STUDY: Northeast Iowa is an agricultural region in an area of karst topography. Previous studies have shown that agricultural chemicals (nitrate and herbicides) have contaminated the Galena aquifer of Ordovician age, which is the source of most domestic water supplies. The contaminant source is generally known; however, knowledge of the flow path of ground water and the chemical processes affecting contamination is limited. Also, the effects of modified agricultural practices on the contaminant levels in surface and ground water need to be monitored.

OBJECTIVES: To further characterize the water quality in the Big Spring Basin and to define several aspects of the hydrologic flow system. Specific objectives include: (1) define ground-water recharge and discharge rates; (2) characterize the quality of small streams that drain into sinkholes; (3) identify the amount and quality of water lost from streams due to seepage; (4) monitor quality and sediment load of water leaving the basin through Big Spring and Roberts Creek; and (5) define geochemical processes that affect surface-water quality.

PROGRESS: The ground-water recharge and discharge rates have been partially defined by measuring stream discharge at about 40 sites in the basin to calculate the amount of water that infiltrates to the aquifer through stream seepage. Ground-water discharge is measured continuously at Big Spring. The quality and quantity of water in Unnamed Creek is monitored to characterize the water flowing directly into sinkholes. Specific conductance, water temperature, and $\mathrm{pH}$ are monitored continuously at both the surface-water discharge point, Roberts Creek above Saint Olaf, and at the ground-water discharge point, Big Spring (fig. 9). Sediment samples are collected at these sites to calculate the sediment load discharged from the basin. Monthly and intermittent samples for the analyses of nutrients and selected herbicides are collected to document changing water quality. A study was conducted in the Deer Creek watershed, a small headwater watershed, to determine how agricultural chemicals are transported to the stream after application. Another study investigated the process of instream herbicide degradation. Data collected during this study have been published in annual U.S. Geological Survey Open-File Reports (Kalkhoff, 1989a; Kalkhoff and Kuzniar, 1991). A report on "Time of Travel and Dispersion in a Selected Reach of Roberts Creek, Clayton County, Iowa" as part of the instream degradation study was published as U.S. Geological Survey 
Water-Resources Investigations Report 91-4145 (Kolpin and Kalkhoff, 1992). Results of the degradation study also are being prepared for publication as a journal article. Results of the study in the Deer Creek watershed are being prepared for publication in a U.S. Geological Survey Water-Resources Investigations Report.
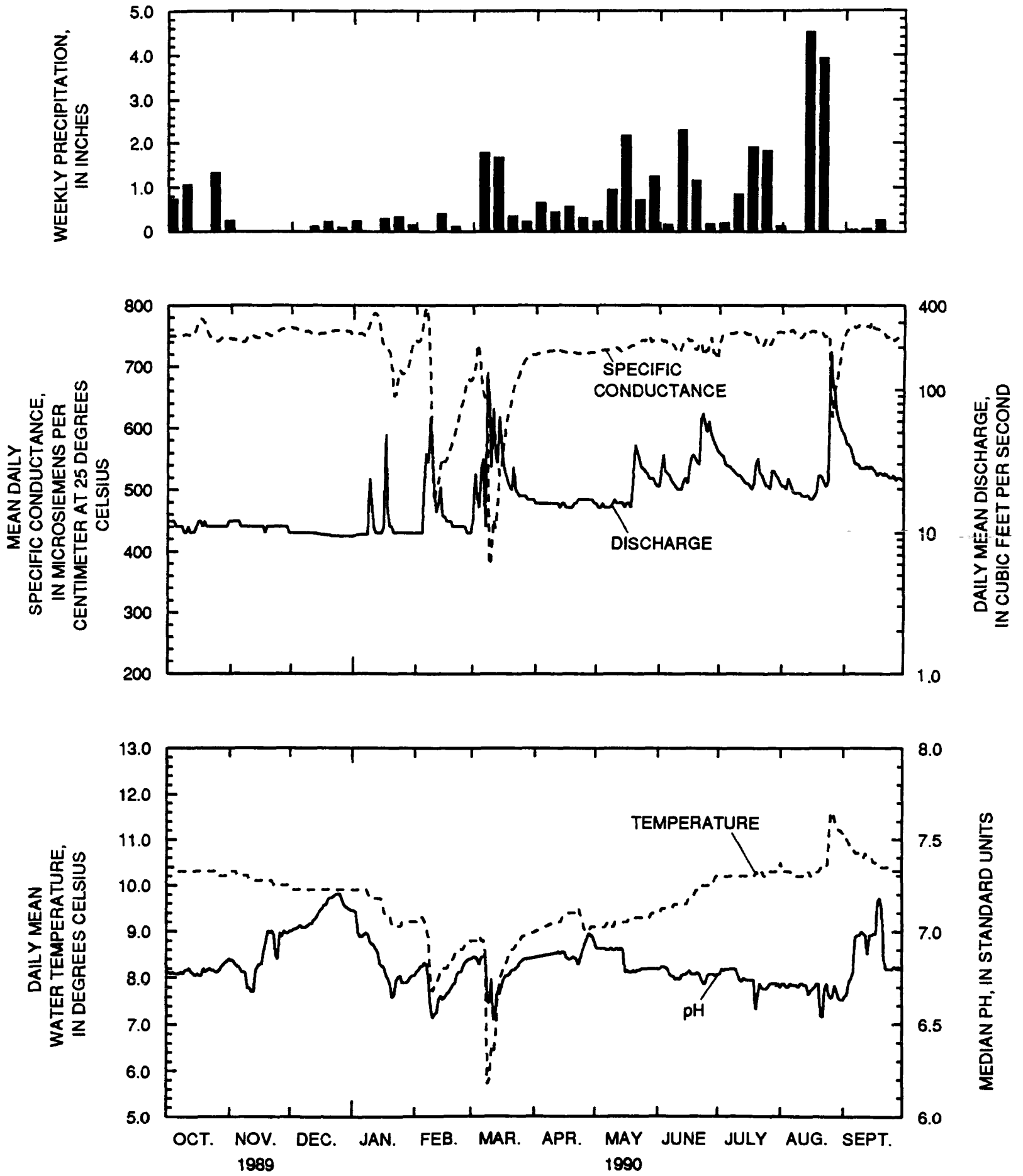

Figure 9. Weekly precipitation, mean daily specific conductance, daily mean discharge, daily mean water temperature, and median pH at Big Spring, Clayton County, lowa, water year 1990. 
MOVEMENT OF NONPOINT-SOURCE AGRICULTURAL CHEMICALS BY THE INTERACTION OF GROUND WATER AND SURFACE WATER IN AN ALLUVIAL AQUIFER (IA 88-061)

PERIOD OF PROJECT: 1988-92

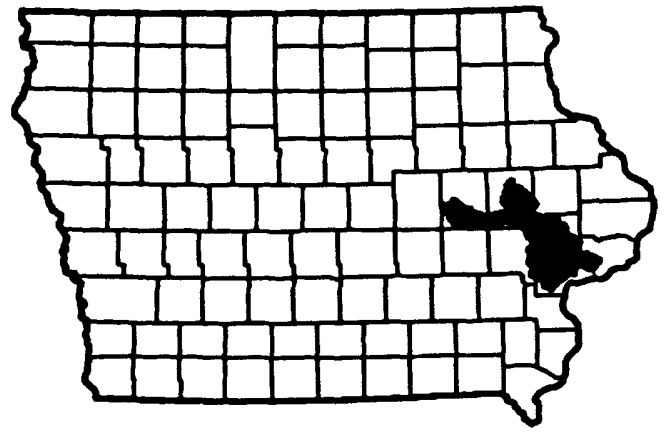

PROJECT CHIEF: P.J. Squillace

STUDY AREA: All or parts of Benton, Cedar, Johnson, Jones, Linn, Muscatine, and Scott Counties, Iowa.

COOPERATING AGENCY: U.S. Geological Survey (Federal program).

NEED FOR STUDY: Herbicides have been detected in rivers for a number of years during runoff and base-flow conditions. The presence of herbicides in rivers during base-flow conditions may indicate a ground-water source. Research is needed to determine how the surface-water quality affects the water quality of the alluvial aquifers and how the water quality of the ground water leaving the alluvial aquifer affects the water quality of the streams.

OBJECTIVE: The objective of this research project is to examine the significance of the exchange of nonpoint sources of contaminants between ground water and surface water by focusing on two questions: (1) to what extent does the water quality of a river affect the water quality of the alluvial aquifer as a result of changes in river stage? and (2) what is the contribution of nonpoint sources of agricultural contaminants to the river by alluvial aquifers during base-flow conditions?

PROGRESS: Three seepage investigations were conducted along the main stem of the Cedar River to determine where agricultural chemicals enter the river during base-flow conditions. The seepage investigations were conducted when the river was in base flow and there was no overland flow into the river. Forty-one wells were installed at a site in the alluvium along the Cedar River, and the water from the wells was sampled 15 times for water-quality properties and constituents. These wells were sampled to determine how agricultural chemicals move in and out of the alluvial aquifer with bank storage. The alluvial-aquifer material was analyzed for organic-carbon content, mineralogy, and particle-size analysis. The data-collection phase of the project has been completed. Figure 10 shows how atrazine in the alluvial aquifer increased near the river during runoff (March 20-22, 1990) due to bank storage of the river water. Other data collected indicate a ground-water source of herbicides to the river during base-flow conditions. As a result of the research conducted during this project, two reports and eight abstracts have been published, five reports are in press, and three reports are in preparation. 
Sampling period February 20-22

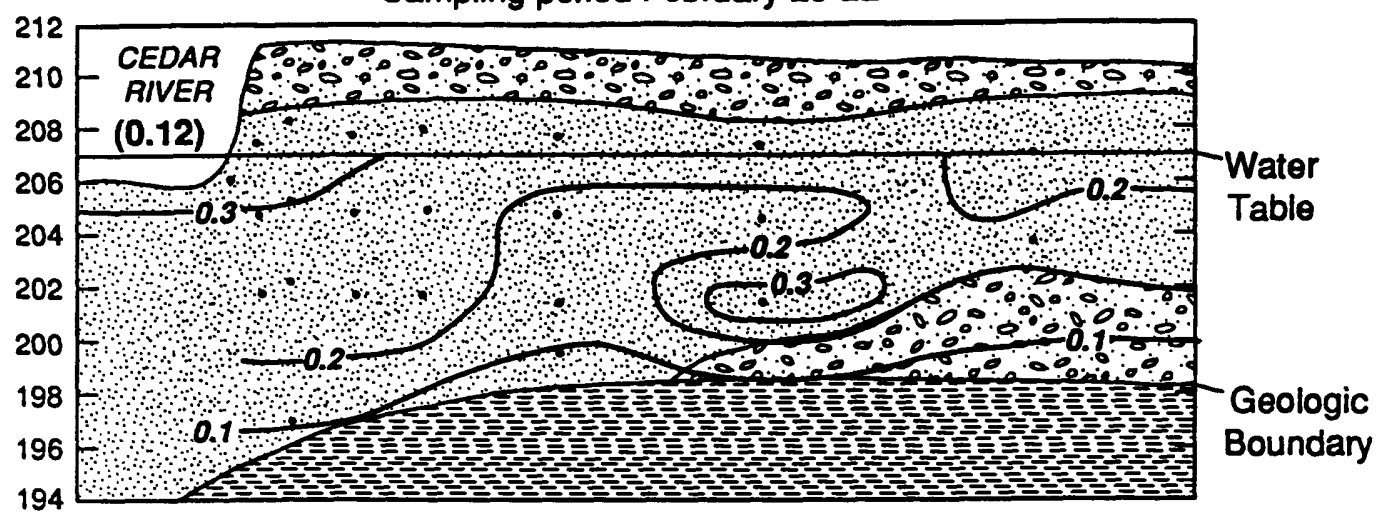

Sampling period March 20-22

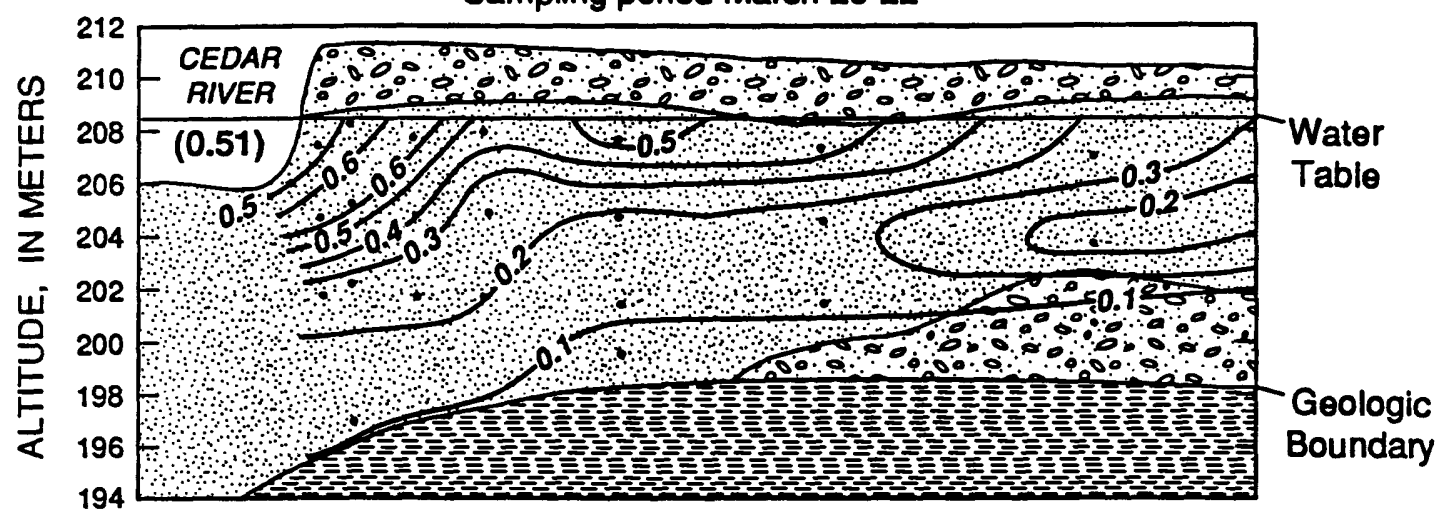

Sampling period April 3-5

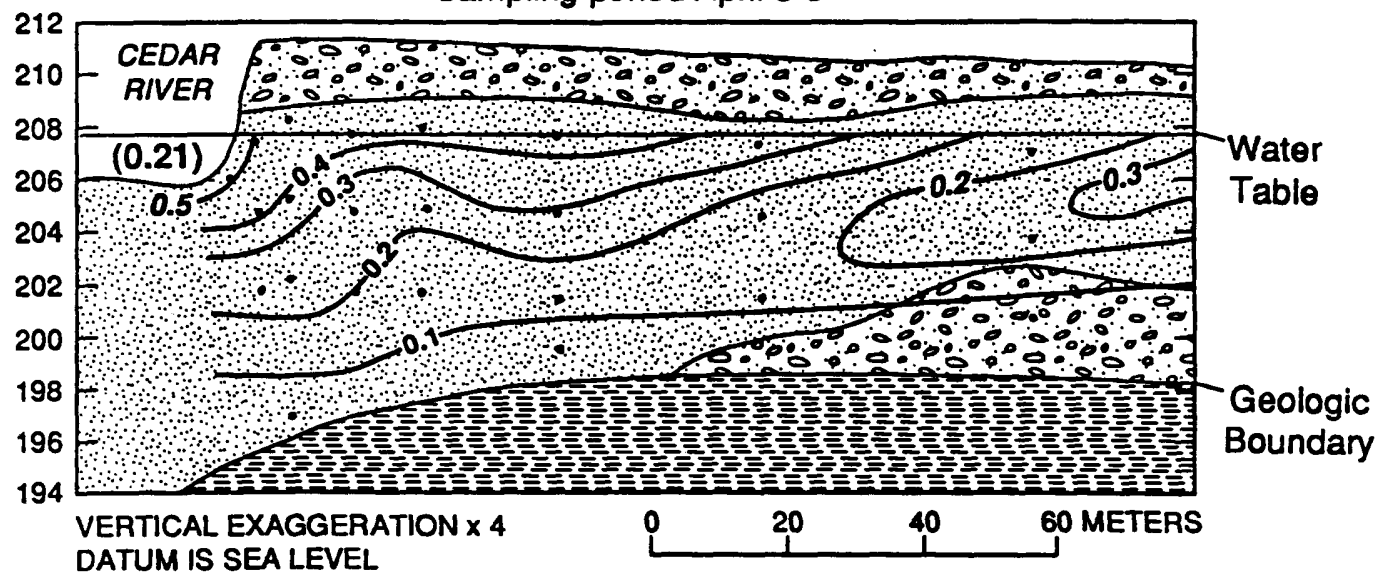

EXPLANATION

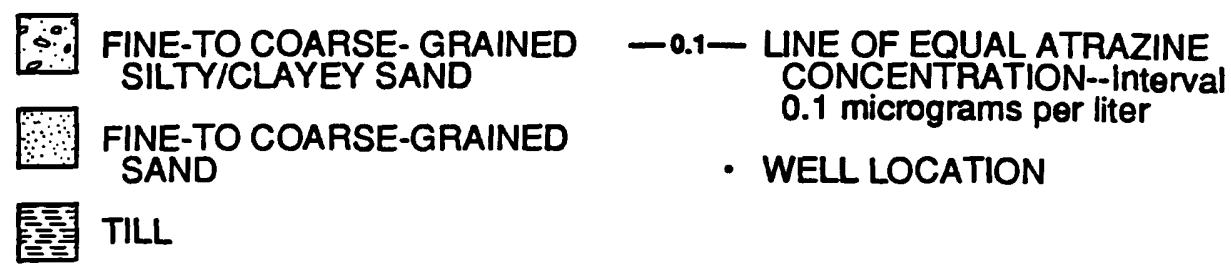

Figure 10. Cross-sectional distribution of atrazine in Cedar River alluvium near Cedar Rapids, lowa, February through April 1990. 
HYDROLOGY OF PRE-ILLINOIAN TILL IN IOWA

\section{(IA 88-063)}

PERIOD OF PROJECT: 1988-92

PROJECT CHIEF: R.C. Buchmiller

STUDY AREA: Linn County

COOPERATING AGENCY: Iowa Department of Natural Resources (Geological Survey Bureau).

NEED FOR STUDY: A great deal of research on the hydrology of Wisconsin glacial till has been done during the last two decades. However, little research has been done on the hydrology of pre-Illinoian glacial till. It is unknown, therefore, whether the hydrology of Wisconsin and pre-Illinoian till differs. In general, glacial till can be divided into two distinct zones: (1) an upper, fractured, weathered zone and (2) a lower, unfractured, unweathered zone. The physics of water movement through pre-Illinoian till needs to be defined--in particular, the relation of fractures in the till to water movement. Changes in the chemistry of water as it moves through pre-Illinoian till needs to be determined, particularly as related to potential contaminants applied or disposed of on the land surface.

OBJECTIVES: The objectives of this study are to determine the physics and chemistry of ground-water movement in pre-Illinoian glacial till.

PROGRESS: Field work began in September 1989 at a site in Linn County where pre-Illinoian glacial till is present. A hydrologic data-collection network, consisting of 22 observation wells and a meteorological station, was installed to describe the hydraulic properties of the till. Continuous water-level recorders were installed in 12 observation wells to monitor water-level fluctuations. A hydrograph from one of these wells is shown in figure 11. A meteorological station consisting of a rain gage, a barometric-pressure sensor, and an air-temperature sensor were installed to monitor climatic conditions. A multiple-constituent water-quality minimonitor was installed to measure temporal changes in specific conductance, water temperature, and $\mathrm{pH}$ of the ground water and to measure the changes that occur due to precipitation. Ten unvented, vibrating-wire, pressure transducers were buried at specific depths in two boreholes to provide an alternate method of collecting water-level and water-temperature measurements. Hydraulic-conductivity measurements, using vented pressure transducers, were conducted in 21 observation wells to record hydraulic-conductivity values. Forty-eight nested piezometers were installed for a tracer-injection study to monitor the three-dimensional movement of solute transport in the shallow, weathered, fractured till. Selected observation wells are sampled periodically for chemical analyses. All monitoring instruments at the study site were connected to digital data loggers. 


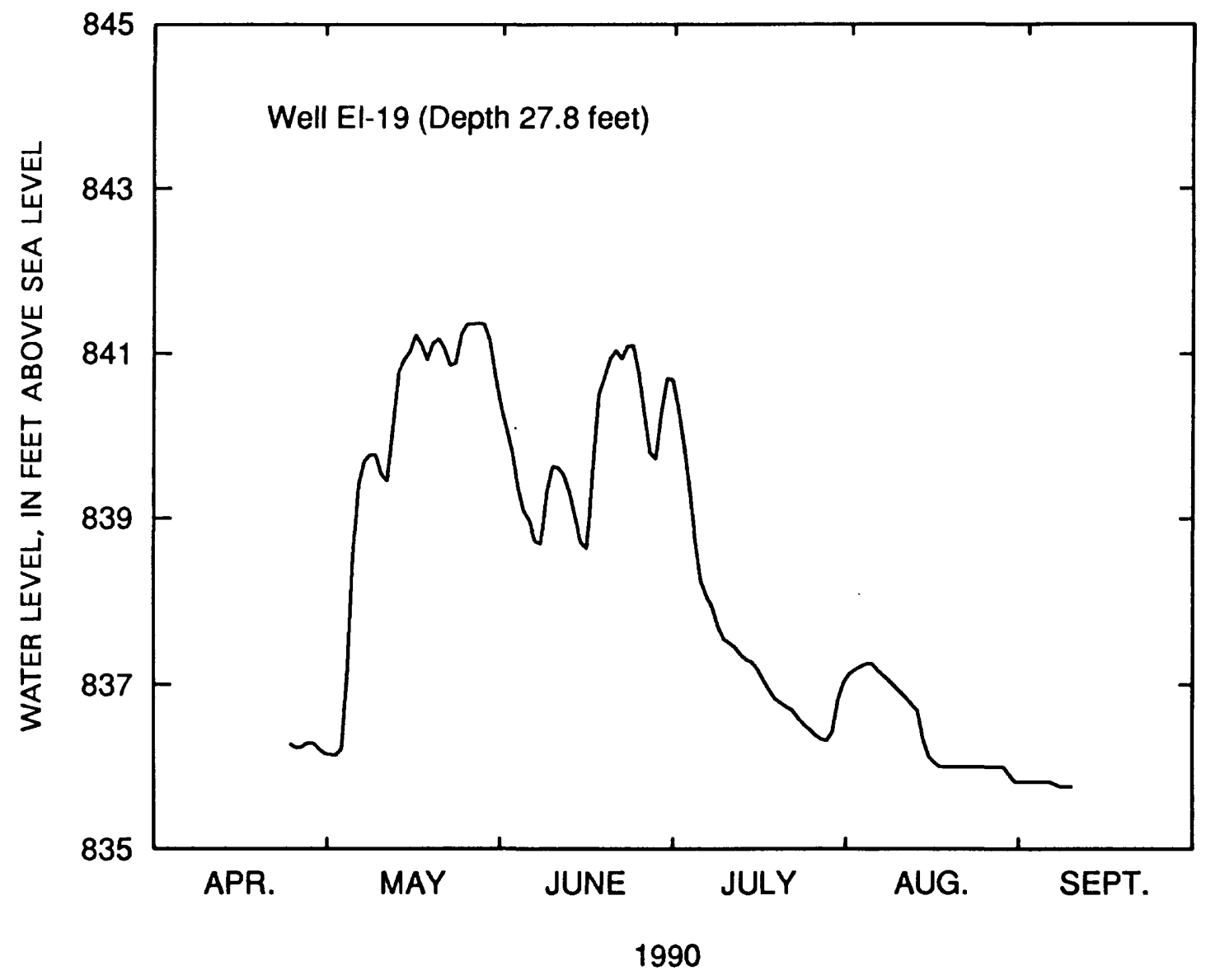

Figure 11. Daily mean water level in continuously monitored observation well El-19, April-September 1990, near Cedar Rapids, lowa. 
ESTIMATING DESIGN FLOOD

DISCHARGES FOR IOWA USING

DRAINAGE-BASIN AND CHANNELGEOMETRY CHARACTERISTICS

\section{(IA 89-064)}

PERIOD OF PROJECT: 1990-92

PROJECT CHIEF: D.A. Eash

STUDY AREA: Statewide

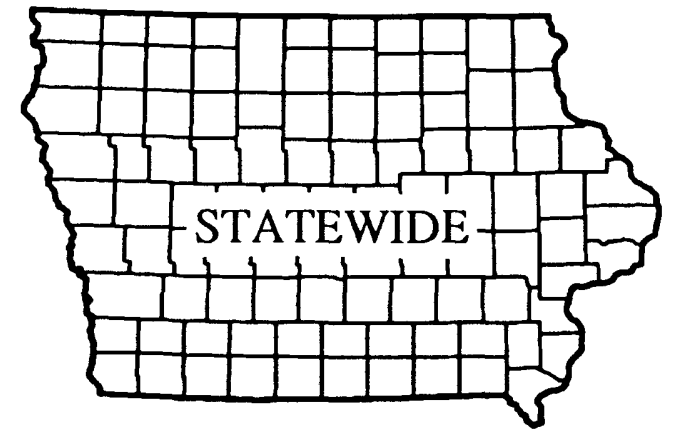

COOPERATING AGENCY: Iowa Department of Transportation (Highway Division, Highway Research Advisory Board).

NEED FOR STUDY: Knowledge of the magnitude and frequency of floods is essential for the economical planning and safe design of bridges, culverts, levees, and for other structures located along streams in Iowa and for the efficient management of flood plains. Two problems are encountered using the current hydrologic regionalization method to estimate design flood discharges for Iowa. First, because regional borders are not well-defined lines, it is difficult to proportion flood estimates for basins located in more than one hydrologic region. Second, because hydrologic regions contain basins with anomalous physiographies, it is difficult to reclassify or to proportion the regional flood estimates.

OBJECTIVES: To further characterize the flood potential for Iowa streams using two flood-estimation methods that are independent of hydrologic regionalization. Specific objectives include: (1) Develop a single set of equations for Iowa that relate measurable basin characteristics to flood discharges of 2-, 5-, 10-, 25-, 50-, and 100-year frequencies; (2) develop a corroborative set of equations for Iowa that relate channel-geometry characteristics to flood discharges of the same frequencies; and (3) obtain an improved understanding of the effect of drainage-basin and stream-channel morphology on flood runoff in Iowa.

PROGRESS: Channel-geometry measurements have been collected, and sediment samples from 170 gaging stations in Iowa have been analyzed for percent silt-clay and median grain size. A geographicinformation-system (GIS) procedure has been developed to quantify 29 drainage-basin characteristics. The GIS procedure uses ARC/INFO software to digitize basin-boundary coverages from 1:250,000-scale topographic maps, to create stream-network coverages from 1:100,000-scale digital line-graph data, and to create elevation-contour coverages from 1:250,000-scale digital-elevation model data. These three basin coverages, along with a long-term, mean annual precipitation coverage and a 2-year, 24-hour precipitation coverage, are processed using software developed by the Iowa District to quantify the 29 basin characteristics. Comparison measurements indicate that the quantification software developed by the Iowa District provides reliable results, and that 10 of 11 key basin characteristics quantified using the GIS procedure are within 10 percent of manual measurements made from the same scale of topographic maps. Basin slope appears to be consistently undermeasured due to limitations inherent in the GSI procedure. Approximately 100 of the 165 basins to 
be used in the basin-characteristics data set have been processed using the GIS procedure. Flood-frequency analyses for the gaging stations being used in the study have been updated through the 1990 water year. A report presenting flood-frequency equations and summary results is planned as a U.S. Geological Survey Water-Resources Investigations Report. 
ANALYSIS OF THE GROUND-WATER FLOW SYSTEM, GEOCHEMISTRY, AND UNDERSEEPAGE IN THE VICINITY OF THE RED ROCK DAM NEAR PELLA, IOWA

(IA 89-065)

PEROID OF PROJECT: 1989-91

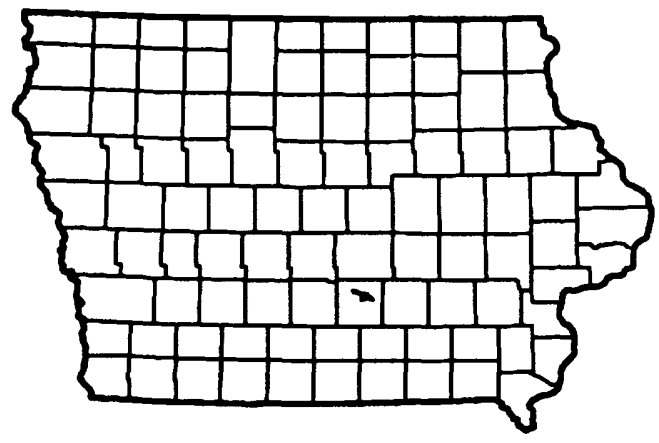

PROJECT CHIEF: K.J. Lucey

STUDY AREA: Marion County

COOPERATING AGENCY: U.S. Army Corps of Engineers.

NEED FOR STUDY: Since the completion of the Red Rock Dam on the Des Moines River in 1969, the U.S. Army Corps of Engineers has been collecting geologic and hydrologic data to monitor underseepage conditions. The bedrock foundation of the dam in the river valley is highly fractured and weathered from dissolution of a basal gypsum zone. Seepage of reservoir water could cause dissolution of the gypsum in the bedrock and threaten the integrity of the earthen structure.

OBJECTIVES: The objectives of this project are to: (1) evaluate the ground-water flow system, geochemistry, and underseepage conditions; (2) determine the flow path of any seepage; and (3) address the potential for dissolution of gypsum from the bedrock foundation.

PROGRESS: An interpretive report, "Analysis of the Ground-Water Flow System, Geochemistry, and Underseepage in the Vicinity of the Red Rock Dam near Pella, Iowa," has been published as U.S. Geological Survey Water-Resources Investigation Report 91-4092 (Lucey, 1991). The report provides an interpretation of hydrologic data collected by the U.S. Army Corps of Engineers and the U.S. Geological Survey from 70 ground-water monitoring wells at the dam site (fig. 12). Chloride-concentration data indicate a hydraulic connection between the reservoir and the ground-water system on the northeast side of the dam. Underseepage occurs through the basal evaporite zone of the St. Louis Limestone and through glacial sand in the northeast bluff. 


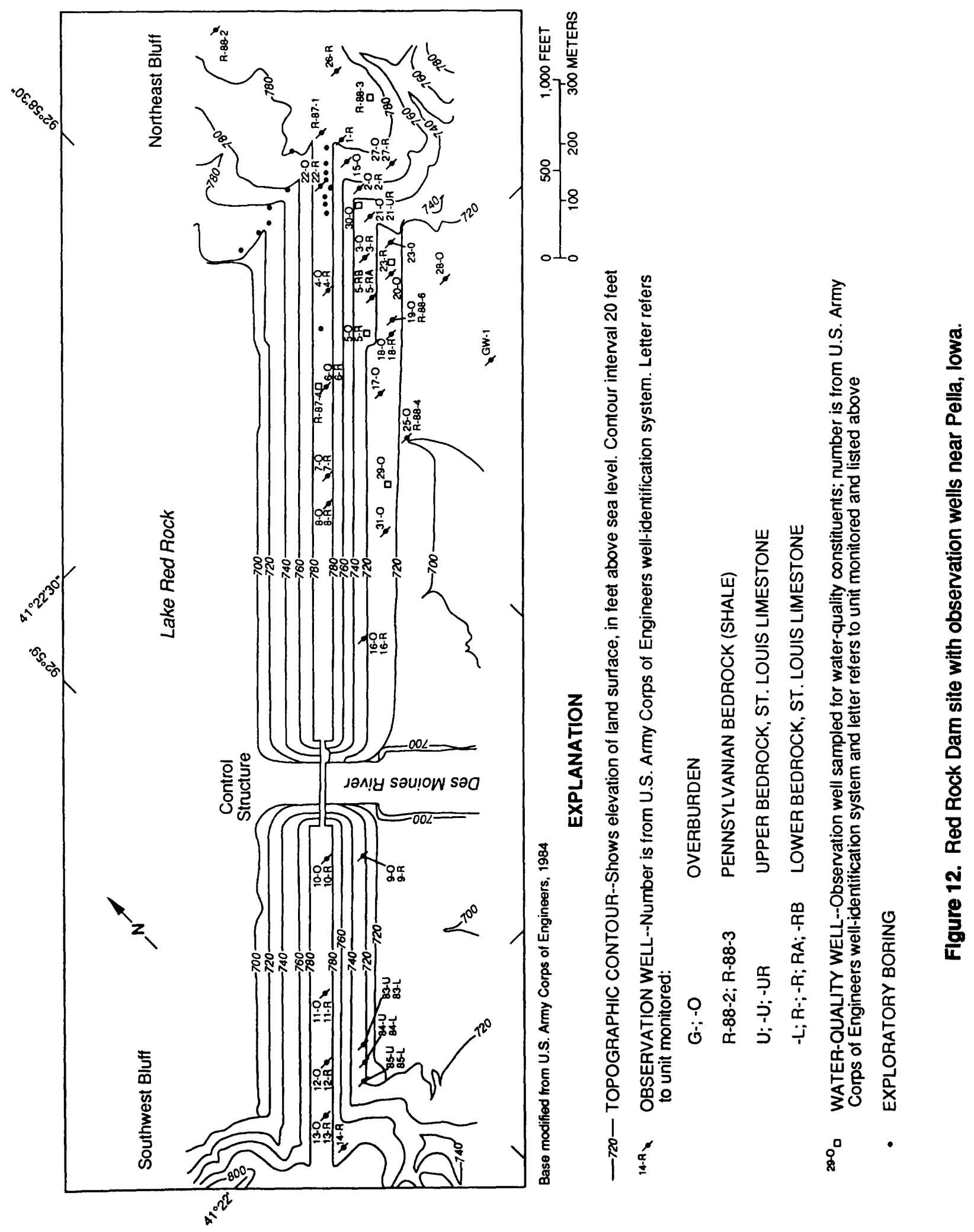


WATER-FLOW PROCESSES AND

RELATED AGRICULTURAL CHEMICAL

LOADINGS IN THE WALNUT CREEK

WATERSHED NEAR AMES, IOWA

(IA 91-067)

PERIOD OF PROJECT: 1991-93

PROJECT CHIEF: P.J. Soenksen

STUDY AREA: Parts of Boone and Story Counties

COOPERATING AGENCY: U.S. Geological Survey (Federal program).

NEED FOR STUDY: The processes governing how agricultural chemicals are transported from points of application to surface- and ground-water resources must be understood in order to minimize contamination of those resources. Agricultural chemicals, such as nitrate, atrazine, alachlor, metolachlor, and metribuzin, are soluble in water to varying degrees, and their transport is thought to be related to the major water-flow processes. Understanding how such factors as physical basin characteristics, land use, agricultural management systems, and meteorological factors affect water-flow processes should increase understanding of agricultural chemical transport.

OBJECTIVES: The Iowa District is working in cooperation with the U.S. Department of Agriculture, Agricultural Research Service (ARS), and the Agricultural Experiment Station (AES) of Iowa State University on the Iowa Management System Evaluation Area (MSEA) project. The following objectives relate to the Iowa District's effort on the Walnut Creek watershed (fig. 13) and are secondary objectives of the Iowa MSEA: (1) define ground-water flow paths and estimate movement in the saturated zone for selected basins; (2) evaluate the ability of the Precipitation-Runoff Modeling System to simulate, at various scales, the major flow processes (water and sediment) and compare with simulations of other models being used; and (3) relate the loadings of agricultural chemicals and sediment in streamflow and tileflow to the major flow processes.

PROGRESS: A brief workplan, with a schedule of activities was prepared. In conjunction with ARS, 65 ground-water wells, 3 surface-water gaging and sampling stations, and 1 surface-water-tileflow gaging and sampling station were installed. Since March 1991, wells have been monitored biweekly and sampled monthly, and surface water and precipitation have been monitored continuously. Approximately 590 water-quality and 350 sediment samples have been collected at surface-water stations. The ARS and AES have monitored and sampled tileflow and meteorological and agricultural contributions, and have worked on field studies. The following coverages were developed for a geographic information system of the watershed: (1) topography, (2) surface drainage, (3) transportation, (4) study sites, and (5) basin and municipal boundaries. A number of ground-water and rainfall samples were collected for oxygen-isotope analyses to help determine the origins of subsurface flow. 


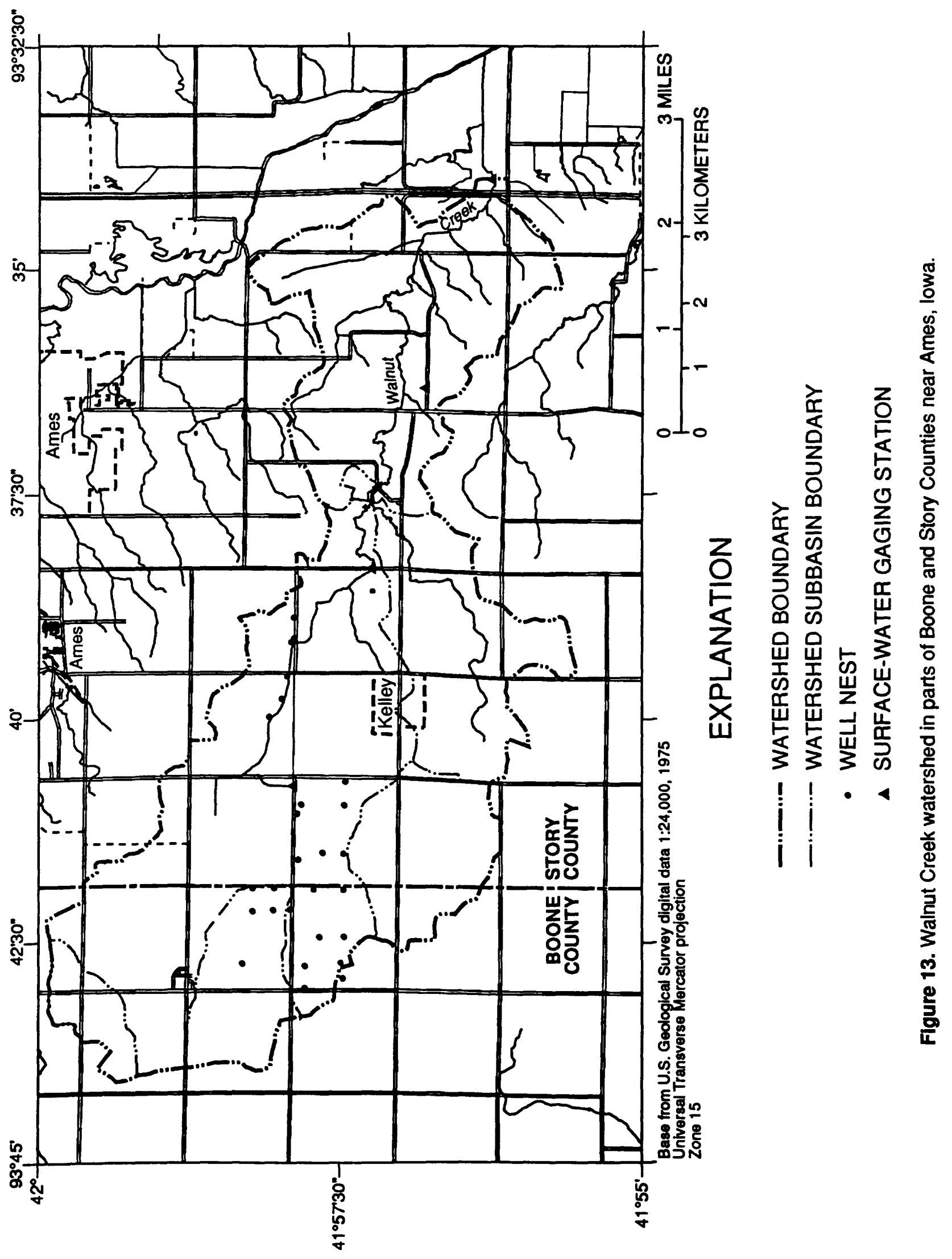




\section{AGRICULTURAL CHEMICALS IN A WATER-SUPPLY RESERVOIR IN SOUTH-CENTRAL IOWA (IA 91-068)}

PERIOD OF PROJECT: 1991-92

PROJECT CHIEF: S.J. Kalkhoff

STUDY AREA: Wayne County

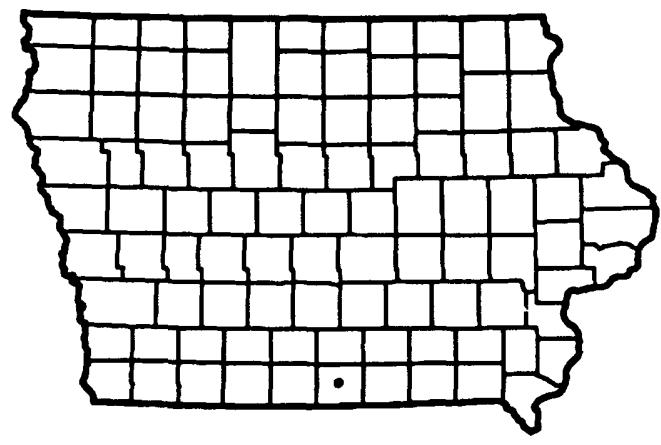

COOPERATING AGENCY: Iowa Department of Natural Resources (Geological Survey Bureau).

NEED FOR STUDY: Previous analyses of water samples from the Corydon Reservoir, used as a source for municipal water supply, indicate that, at times, agricultural-chemical concentrations exceed U.S. Environmental Protection Agency Maximum Contaminant Levels for drinking water. To reduce agricultural-chemical runoff, several Federal, State, and local agencies (U.S. Environmental Protection Agency; U.S. Department of Agriculture's Soil Conservation Service and Agricultural Stabilization and Conservation Service; Iowa State University Cooperative Extension Service; Iowa Department of Agriculture and Land Stewardship; and the City of Corydon) have provided funding and personnel to assist the landowners in the Corydon Reservoir watershed to modify their agricultural practices and to implement conservation practices. This is intended to reduce the input of nitrates, herbicides, and sediment into the reservoir. There is a need to monitor the water quality of the Corydon Reservoir to assess the affect of these conservation practices.

OBJECTIVES: To monitor the effect of changing land-management practices on the quality of water in the Corydon Reservoir, Wayne County, Iowa.

PROGRESS: An initial areal reservoir survey was completed in September 1990 to assess the variability of the water quality in the reservoir and to select representative sites for monthly sampling. The three sites selected have been sampled monthly for concentrations of nutrients, selected herbicides, total solids, bacteria, and chlorophyll. The reservoir level is recorded continuously to determine the reservoir storage and discharge from the watershed. Precipitation quality and quantity are being determined to document atmospheric input of herbicides into the watershed. 
PERIOD OF PROJECT: 1991-92

PROJECT CHIEF: R.C. Buchmiller

STUDY AREA: Statewide

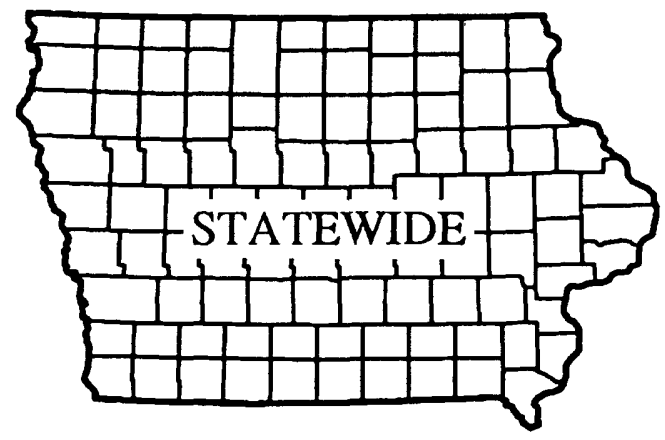

COOPERATING AGENCY: University of Iowa Hygienic Laboratory.

NEED FOR STUDY: Many of the pesticide ingredients used extensively in the cornand soybean-growing areas of the Midwest contain ingredients referred to on product labels as inert ingredients. Testing of raw product formulations has determined that the inert ingredients may contain chemicals such as volatile organic chemicals. Many volatile organic chemicals are regulated under provisions of the Safe Drinking Water Act. Therefore, there is a need to determine the effects of volatile organic compounds contained in pesticide formulations on ground-water quality.

OBJECTIVES: The primary objectives of this project are to determine if significant quantities of volatile organic compounds are present in pesticide formulations that are being applied to Iowa corn and soybean fields and to determine if these chemicals are entering the ground-water system beneath the fields. Samples of pesticide formulations will be obtained for analysis of volatile organic compounds. Field samples from areas where monitoring equipment is in place will be obtained prior to application and after application for analysis to compare with results from the product sampling. Sites that have detectable concentrations of volatile organic compounds will be studied in greater detail.

PROGRESS: Four sites were selected for collection of field samples: Deer Creek in Clayton County; the Nashua till site in Floyd County; and the Ames till site and Walnut Creek in Story County. More than 300 samples were collected from the sites prior to application and at selected dates after application. Samples of the product formulations that were applied to the sites were obtained. Several volatile organic compounds were found in the product formulations in percent amounts. Small concentrations of several volatile organic compounds were detected in field samples both before and after application. Plans for modifying the field-sampling procedure during 1992 are underway. 
DETERMINATION OF STORM-WATER RUNOFF QUALITY IN DAVENPORT, IOWA

(IA 91-070)

PERIOD OF PROJECT: 1991-94

PROJECT CHIEF: K.J. Lucey

STUDY AREA: Scott County

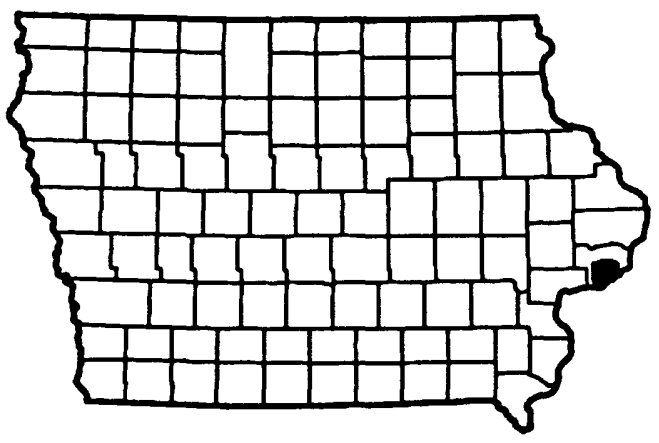

COOPERATING AGENCY: City of Davenport

NEED FOR STUDY: Cumulative constituent loads associated with urban runoff can have significant effects on the water quality of receiving streams. If the source of possible contaminants can be determined, management controls can be implemented. The quantity and quality of storm-water runoff will be characterized for five major land uses: (1) residential, (2) commercial, (3) industrial, (4) agricultural, and (5) urban open space. Annual contaminant loads for 12 constituents for each land-use type will be determined. The data collected will satisfy the requirements of the U.S. Environmental Protection Agency's National Pollution Discharge Elimination System permit application for discharges from municipal storm-sewer systems.

OBJECTIVE: The objective of the study is to characterize the quantity and the quality of storm water in streams and channels draining five areas of specific land uses. The data will be used to determine mean concentrations and loads of selected constituents in storm-water discharges for the city of Davenport for each period of runoff.

PROGRESS: Geographic-information-system (GIS) coverages for land-use data, outfall locations, outfall drainage areas, storm-sewer network, roads, topographic contours, and streams were compiled. The coverages can be used to determine the percentages of various land-use types contributing to a drainage area. Existing hydrologic data for the streams receiving urban runoff were compiled. Five monitoring sites were selected that will characterize urban runoff from the various land uses. A surface-water gaging station and a precipitation collector will be installed and maintained for a 2-year period to gather data from three storms at each site. Water-quality sampling will be done during the first 3 hours of each storm. 


\section{EFFECT OF LAND-USE CHANGES ON STREAM SEDIMENT IN THE SNY MAGILL WATERSHED, CLAYTON COUNTY, IOWA \\ (IA 92-072)}

PERIOD OF PROJECT: 1992-96

PROJECT CHIEF: S.J. Kalkhoff

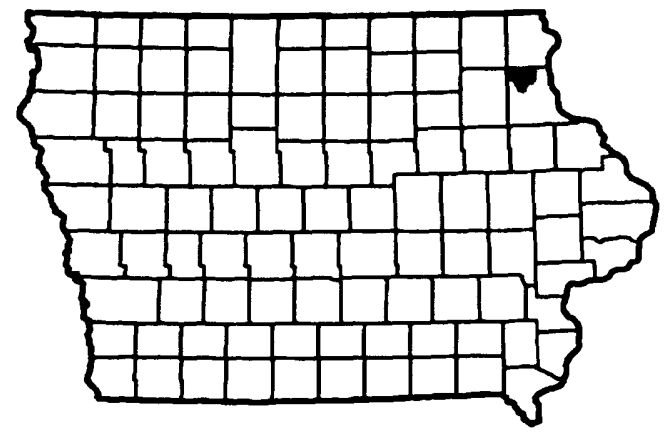

STUDY AREA: Clayton County

COOPERATING AGENCY: Iowa Department of Natural Resources (Geological Survey Bureau).

NEED FOR STUDY: Stream sediment continues to be a major problem in rivers and streams throughout the corn- and soybean-growing region of the midcontinental United States. Measurements of the sediment yield of Mississippi River tributaries in Iowa indicate that sediment losses are between 500 ton/acre in north-central Iowa to 1,500 ton/acre in east-central Iowa. Sediment yield from tilled agricultural land has been shown to be 20 times greater than pastured land and hundreds of times greater than forested land. Increases in stream sediment can cause the loss of productive topsoil, channel instability, altered stream biology, and can limit the recreational and aesthetic value of the stream's environment. Public and Congressional concern for the contamination of ground- and surface-water supplies has prompted the agricultural community to re-evaluate present farming methods. The U.S. Department of Agriculture has been charged with developing and implementing best-management practices intended to lessen the environmental effects of present agricultural land-use practices. These best-management practices will be implemented on the local level through a series of farm demonstration projects located in selected hydrologic units. To determine the effectiveness of best-management practices, monitoring of the changes in stream sediment is needed.

OBJECTIVES: The principal objective of the project is to determine the effectiveness of land-use practices implemented in the Sny Magill watershed to reduce stream-sediment yield. Specific project objectives include: (1) evaluation of the stream-sediment yield from a basin where present agricultural practices are continued; (2) evaluation of the effectiveness of riparian buffer strips in reducing stream-sediment yield; and (3) evaluation of the effects of best-management practices and sediment controls in reducing stream-sediment yield.

PROGRESS: The project began October 1991. 
INVESTIGATION OF SCOUR SUSCEPTIBILITY AT BRIDGES IN IOWA

(IA 92-071)

PERIOD OF PROJECT: 1992-94

PROJECT CHIEF: E.E. Fischer

STUDY AREA: Statewide

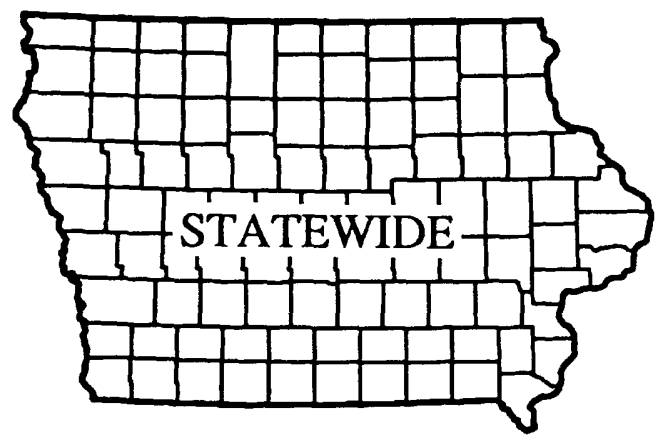

COOPERATING AGENCY: Iowa Department of Transportation.

NEED FOR STUDY: The Federal Highway Administration has requested that the Iowa Department of Transportation (IDOT) and the State's counties and cities assess the scour-related integrity of bridges in Iowa because of several scour-related bridge failures in other parts of the country. In 1990 , the IDOT initiated a bridge-scour evaluation of more than 2,000 bridges over water in the State's primary highway system. Transportation officials in Iowa's counties and cities also have begun to evaluate more than 21,000 bridges in the secondary highway system. To assist them in their scour review, IDOT has requested that the U.S. Geological Survey (USGS) provide an assessment of regional scour susceptibility for bridges in Iowa.

OBJECTIVE: The objective of the Iowa bridge scour-susceptibility project is to provide regional information and understanding of scour at bridge sites throughout the State. Working in cooperation with the IDOT, the USGS will assess scour susceptibility at about 100 bridge sites throughout the State by field inspection, select between 8 and 15 sites to collect data for more detailed evaluations of scour susceptibility, and make scour analyses. The USGS will attempt also to collect measurements of scour during several high-flow events.

PROGRESS: The project began October 1991. 


\section{SELECTED REFERENCES}

[Reports prepared since fiscal year 1984]

Bowman, P.R., 1991, Hydrologic data for a study of pre-Illinoian glacial till in Linn County, Iowa, water year 1990: U.S. Geological Survey Open-File Report 91-459, 76 p.

Buchmiller, R.C., 1986a, Iowa joins in monitoring acid rain: Iowa Conservationist, v. 45 , no. 9 , p. $28-29$.

1986b, Hydrologic reconnaissance and summary of existing data on surface and ground water in the Missouri River valley in Woodbury and Monona Counties, Iowa, 1985: U.S. Geological Survey Open-File Report 86-144, 21 p.

1986c, Use of the gas chromatograph-flame ionization dectector to identify organic substances, in Ragone, S.E., ed., U.S. Geological Survey Program on Toxic Waste--Ground-Water Contamination, 2nd, Cape Cod, Maine, 1985, Fiscal Year 1986 Program Overview and Selected Abstracts: U.S. Geological Survey Open-File Report 86-481, p. F17.

1988a, A strategy for data collection and modeling the Missouri River alluvial aquifer in Woodbury and Monona Counties, Iowa: U.S. Geological Survey Open-File Report 87-452, $16 \mathrm{p}$.

1988b, U.S. Geological Survey ground-water studies in Iowa: U.S. Geological Survey Open-File Report 88-146, 2 p.

1989 , Screening of ground water samples for volatile organic compounds using a portable gas chromatograph: Ground Water Monitoring Review, v. 9, no. 3, p. 126-130.

Buchmiller, R.C., Gaillot, Gary. and Soenksen, P.J., 1985, Water resources of north-central Iowa: Iowa Geological Survey Water Atlas 7, $93 \mathrm{p}$.

Buchmiller, R.C., Squillace, P.J., and Drustrup, R.D., 1988, Iowa ground-water quality, in National water summary 1986--Selected hydrologic events and ground-water quality: U.S. Geological Survey Water-Supply Paper
2325, p. 251-258.

Burkart, M.R., 1991, U.S. Geological Survey--mid-continent herbicide initiative, President's Water-Quality Initiative--A Market Emphasis, in Proceedings of the American Society of Agricultural Engineers, International Summer Meeting: Albuquerque, New Mexico, 1991, p. 18-20.

1991, Multiscale research on agrichemicals in water resources of the mid-continental United States, in Abstracts of the Proceedings of Midwest Ground Water Conference: Indianapolis, Indiana, 1991, p. 10-11.

in press, Hyrdologic research on agrichemicals in the mid-continental United States, in Proceedings of Water Quality Workshop for Eastern Europe: Poznan, Poland, 1991.

Burkart, M.R., and Buchmiller, R.C., 1990, Regional evaluation of hydrologic factors and effects of pumping, St. Peter-Jordan aquifer, Iowa: U.S. Geological Survey Water-Resources Investigations Report 90-4009, 44 p.

Burkart, M.R., Onstad, C.A., and Bubenzer, G.D., 1990, Research on agrichemicals in water resources: EOS, Transactions of the American Geophysical Union, v. 71, no. 29, p. 980-988.

Burkart, M.R., and Ragone, S.E., 1989, Planned studies of herbicides in ground and surface water in the mid-continental United States, in International Symposium on Integrated Approaches to Water Pollution Problems: Lisbon, Portugal, 1989, v. 3, p. 475-477.

Burkart, M.R., Ragone, S.E., Thurman, E.M., and Perry, C.A., 1989, Planned studies of herbicides in ground and surface water in the midcontinental United States, in Mallard, G.E., and Ragone, S.E., eds., U.S. Geological Survey Toxic Substances Hydrology Program, Proceedings of the Technical Meeting, Phoenix, Arizona, 1988: U.S. Geological Survey Water-Resources Investigations Report 88-4220, p. 445-452. 
Clark, M.L., and Thamke, J.N., 1988, Estimated water use in Iowa, 1985: U.S. Geological Survey Open-File Report 87-704, 28 p.

Detroy, M.G., 1986, Areal and vertical distribution of nitrate and herbicides in the Iowa River alluvial aquifer, Iowa County, Iowa, in Agricultural Impacts on Ground Water Conference, 1986, Proceedings: National Water Well Association, p. 381-398.

1989, Seasonal variation and vertical distribution and movement of herbicides and nitrate in unsaturated and saturated alluvium underlying a cropped field, Iowa County, Iowa, in Mallard, G.E., and Ragone, S.E., eds., U.S. Geological Survey Toxic Substances Hydrology Program, Proceedings of the Technical Meeting, Phoenix, Arizona, 1988: U.S. Geological Survey Water-Resources Investigations Report 88-4220, p. 634.

Detroy, M.G., Clark, M.L., Holub, M.A., and Hunt, P.K.B., 1990, Water quality of alluvial aquifers, Carroll and Guthrie Counties, Iowa, with emphasis on the occurrence of nitrates and pesticides: U.S. Geological Survey Water-Resources Investigations Report 89-4186, 52 p.

Detroy, M.G., Hunt, P.K.B., and Holub, M.A., 1988a, Ground-water-quality monitoring in Iowa--Nitrate and pesticides in shallow aquifers: U.S. Geological Survey WaterResources Investigations Report 88-4123, 31 p.

$1988 \mathrm{~b}$, Ground-water-quality monitoring in Iowa--Nitrate and pesticides in shallow aquifers, in Agricultural Impacts on Ground Water Conference, 1988, Proceedings: National Water Well Association, p. 255-278.

Detroy, M.G., and Kuzniar, R.L., 1988, Occurrence and distribution of nitrate and herbicides in the Iowa River alluvial aquifer, Iowa--May 1984 to November 1985: U.S. Geological Survey Water-Resources Investigations Report 88-4117, 93 p.

Eash, D.A., and Heinitz, A.J., 1991, Floods in the Nishnabotna River basin, Iowa: U.S.
Geological Survey Open-File Report 91-171, $118 \mathrm{p}$.

Goldberg, M.C., Cunningham, K.M., and Squillace, P.J., 1991a, Photolytic degradation of atrazine in the Cedar River, Iowa, and its tributaries, in Mallard, G.E., and Aronson, D.A., compilers, U.S. Geological Survey Toxics Substances Hydrology Program, Abstracts of the Technical Meeting, Monterey, California, 1991: U.S. Geological Survey Open-File Report, 91-88, p. 18.

1991b, Photolytic degradation of atrazine in the Cedar River, Iowa, and its tributaries, in Mallard, G.E., and Aronson, D.A., eds., U.S. Geological Survey Toxics Substances Hydrology Program, Proceedings of the Technical Meeting, Monterey, California, 1991: U.S. Geological Survey WaterResources Investigations Report 91-4034, p. 232-239.

Goolsby, D.A., Thurman, E.M., and Kolpin, D.W., 1990, Geographic and seasonal distribution of herbicides in streams of the upper midwestern United States, in Abstracts of the Northern Rocky Mountain Water Congress, Butte, Montana, 1990.

1991a, Geographic and temporal distribution of herbicides in surface waters of the upper midwestern United States, 1989-90, in Mallard, G.E., and Aronson, D.A., compilers, U.S Geological Survey Toxic Substances Hydrology Program, Abstracts of the Technical Meeting, Monterey, California, 1991: U.S. Geological Survey Open-File Report 91-88, p. 10.

1991b, Geographic and seasonal distribution of herbicides in streams of the midwestern United States, in Ritter, W.F., ed., Irrigation and Drainage, Proceedings of the 1991 National Conference of the American Society of Civil Engineers: Honolulu, Hawaii, 1991, p. 17-23. 
1991c, Geographic and temporal distribution of herbicides in surface waters of the upper midwestern United States, 1989-1990, in Mallard, G.E. and Aronson, D.A., eds., U.S. Geological Survey Toxic Substances Hydrology Program, Proceedings of the Technical Meeting, Monterey, California, 1991: U.S. Geological Survey WaterResources Investigations Report 91-4034, p. 183-188.

Goolsby, D.A., Thurman, E.M., Kolpin, D.W., and Detroy, M.G., 1989, A reconnaissance for triazine herbicides in surface waters of the upper midwestern United States, in Pederson, G.L., and Smith, M.M., compilers, U.S. Geological Survey Second National Symposium on Water Quality, Abstracts of the Technical Sessions, Orlando, Florida, 1989: U.S. Geological Survey Open-File Report 89-409, p. 31.

Hansen, R.E., 1985, Bedrock topography of central Iowa: U.S. Geological Survey Miscellaneous Geologic Investigations Map I-1609, scale 1:125,000, 2 sheets.

1986, Bedrock topography of northwest Iowa: U.S. Geological Survey Miscellaneous Geologic Investigations Map I-1726, scale $1: 125,000,2$ sheets.

Hansen, R.E., and Runkle, D.L., 1986, Bedrock topography of west-central Iowa: U.S. Geological Survey Miscellaneous Geologic Investigations Map I-1688, scale 1:125,000, 2 sheets.

Hansen, R.E., Thompson, C.A., and VanDorpe, P.E., 1992, Availability and quality of water from the alluvial, glacial-drift, and Dakota aquifers and water use in southwest Iowa: U.S. Geological Survey Water-Resources Investigations Report 91-4156, $187 \mathrm{p}$.

Heinitz, A.J., 1985a, Floods in south-central Iowa: U.S. Geological Survey Open-File Report 85-100, $95 \mathrm{p}$.

1985b, Floods of June-July 1982, in Iowa: U.S. Geological Survey Open-File Report 85-151, 18 p.

1985 c, Discharge ratings for control gates at Mississippi River lock and dam 11,
Dubuque, Iowa: U.S. Geological Survey Water-Resources Investigations Report 85-4105, 43 p.

1985d, Discharge ratings for control gates at Mississippi River lock and dam 14, Le Claire, Iowa: U.S. Geological Survey Water-Resources Investigations Report 85-4261, 38 p.

1986a, Floods in the Floyd River basin, Iowa: U.S. Geological Survey Open-File Report 86-476, 61 p.

$1986 \mathrm{~b}$, Discharge ratings for control gates at Mississippi River lock and dam 13, Fulton, Illinois: U.S. Geological Survey WaterResources Investigations Report 86-4134, 43 p.

1986c, Discharge ratings for control gates at Mississippi River lock and dam 12, Bellevue, Iowa: U.S. Geological Survey WaterResources Investigations Report 86-4135, 43 p.

1986d, Discharge ratings for control gates at Mississippi River lock and dam 16, Muscatine, Iowa: U.S. Geological Survey Water-Resources Investigations Report 86-4136, 36 p.

1986 e, Discharge ratings for control gates at Mississippi River lock and dam 22, Saverton, Missouri: U.S. Geological Survey Water-Resources Investigations Report 86-4137, 37 p.

1987a, Discharge ratings for control gates at Mississippi River lock and dam 18, Gladstone, Illinois: U.S. Geological Survey Water-Resources Investigations Report $87-4110,44$ p.

1987b, Discharge ratings for control gates at Mississippi River lock and dam 17, New Boston, Illinois: U.S. Geological Survey Water-Resources Investigations Report 87-4165, 44 p.

$1987 \mathrm{c}$, Discharge ratings for control gates at Mississippi River lock and dam 20, Canton, Missouri: U.S. Geological Survey WaterResources Investigations Report 87-4149, 36 p. 
1987d, Discharge ratings for control gates at Mississippi River lock and dam 21, Quincy, Illinois: U.S. Geological Survey WaterResources Investigations Report 87-4155, 44 p.

Horick, P.J., and Soenksen, P.J., 1989, Water resources of northeast Iowa: Iowa Department of Natural Resources Water Atlas 8, $133 \mathrm{p}$.

Hunt, P.K.B., and Detroy, M.G., 1987, Ground-water-quality monitoring in Iowa--Nitrate and pesticides in shallow aquifers, in FOCUS Conference on Midwestern Ground Water Issues, 1987, Proceedings: National Water Well Association, p. 591-612.

Hunt, P.K.B., and Runkle, D.L., 1985, Ground-water data for the alluvial, buried channel, basal Pleistocene and Dakota aquifer in west-central Iowa: U.S. Geological Survey Open-File Report 84-819, $168 \mathrm{p}$.

Kalkhoff, S.J., 1989a, Hydrologic data for the Big Spring basin, Clayton County, Iowa, water year 1988: U.S. Geological Survey Open-File Report 89-230, 44 p.

$1989 \mathrm{~b}$, Contamination of ground and surface water by agricultural chemicals, Big Springs basin, Clayton County, Iowa, in Pederson, G.L., and Smith, M.M., compilers, U.S. Geological Survey Second National Symposium on Water Quality, Abstracts of the Technical Sessions, Orlando, Florida, 1989: U.S. Geological Survey Open-File Report 89-409, p. 43.

$1989 c$, Quality of surface water discharged from the Big Spring basin, Clayton County, Iowa: The Journal of the Iowa Academy of Science, Program Abstracts, v. 96, no. 1, p. A23.

in press, Effect of agricultural chemicals on water quality of Corydon reservoir, Corydon, Iowa: The Regional Lake Management Conference (Program Abstracts).

Kalkhoff, S.J., and Kuzniar, R.L., 1991, Hydrologic data for the Big Spring basin,
Clayton County, Iowa, water year 1989: U.S. Geological Survey Open-File Report 91-63, $66 \mathrm{p}$.

Karsten, R.A., 1985, Iowa--A summary of water-resources activities of the United States Department of the Interior Geological Survey: Iowa City, Iowa, U.S. Geological Survey Report, 46 p.

1990, A summary of water-resources activities of the U.S. Geological Survey in Iowa, fiscal year 1990: Iowa City, Iowa, U.S. Geological Survey Open-File Report 90-583, $49 \mathrm{p}$.

Karsten, R.A., and Burkart, M.R., 1985, Iowa ground-water resources, in National water summary 1984--Hydrologic events, selected water-quality trends, and ground-water resources: U.S. Geological Survey Water-Supply Paper 2275, p. 211-216.

Karsten, R.A., and Waite, P.J., 1987, Water year 1986 in Iowa--Precipitation and water resources: U.S. Geological Survey Open-File Report 87-681, 18 p.

Kolpin, D.W., 1989, Indicators of pesticides in shallow aquifers in Iowa: The Journal of the Iowa Academy of Science, Program Abstracts, v. 96 no. 1, p. A22.

Kolpin, D.W., and Burkart, M.R., 1989, Indicators of pesticides in shallow aquifers in Iowa, in Pederson, G.L., and Smith, M.M., compilers, U.S. Geological Survey Second National Symposium on Water Quality, Abstracts of the Technical Sessions, Orlando, Florida, 1989: U.S. Geological Survey Open-File Report 89-409, p. 47.

1990, Indicators of pesticides in shallow aquifers in Iowa, in Abstracts of the Information Exchange on Models and Data Needs Relating to the Impact of Agricultural Practices on Water Quality Workshop: Proceedings of the U.S. Geological Survey, Agriculturai Research Service, and Soil Conservation Service, p. 87. 
1991, Work plan for regional reconnaissance for selected herbicides and nitrate in ground water of the mid-continental United States, 1991: U.S. Geological Survey Open-File Report 91-59, 18 p.

Kolpin, D.W., and Kalkhoff, S.J., 1992, Time of travel and dispersion in a selected reach of Roberts Creek, Clayton County, Iowa: U.S. Geological Survey Water-Resources Investigations Report 91-4145, 16 p.

Lara, O.G., 1987, Method for estimating the magnitude and frequency of floods at ungaged sites on unregulated rural streams in Iowa: U.S. Geological Survey Water-Resources Investigations Report 87-4132, 34 p.

Lara, O.G., and Eash, D.A., 1987, Floods in Iowa--Stage and discharge: U.S. Geological Survey Open-File Report 87-382, 793 p.

Lara, O.G., and Matthes, W.J., 1986, The sedigraph as an alternative method to the pipet, in Federal Interagency Sedimentation Conference, 4th, 1986: Proceedings, v. 1, p. 1-12.

Lara, O.G., and Soenksen, P.J., 1986, Iowa surface-water resources, in National water summary 1985--Hydrologic events and surface-water resources: U.S. Geological Survey Water-Supply Paper 2300, p. 229-236.

Liszewski, M.J., and Squillace, P.J., 1991, The effect of surface-water and ground-water exchange on the transport and storage of atrazine in the Cedar River, Iowa, in Mallard, G.E., and Aronson, D.A., compilers, U.S. Geological Survey Toxic Substances Hydrology Program, Abstracts of the Technical Meeting, Monterey, California, 1991: U.S. Geological Survey Open-File Report 91-88, p. 12.

1991, The effect of surface-water and ground-water exchange on the transport and storage of atrazine in the Cedar River, Iowa, in Mallard, G.E. and Aronson, D.A., eds., U.S. Geological Survey Toxics Substances Hydrology Program, Proceedings of the Technical Meeting, Monterey, California, 1991: U.S. Geological
Survey Water-Resources Investigations Report 91-4034, p. 195-202.

Lucey, K.J., 1991, Analysis of the ground-water flow system, geochemistry, and underseepage in the vicinity of the Red Rock dam, near Pella, Iowa: U.S. Geological Survey Water-Resources Investigations Report 91-4092, $68 \mathrm{p}$.

Majure, J.J., and Soenksen, P.J., 1991, Using a geographic information system to determine physical basin characteristics for use in flood frequency equations, in Balthrop, B.H., and Terry, J.E., eds., U.S. Geological Survey National Computer Technology Meeting, Phoenix, Arizona, November 14-18, 1988: U.S. Geological Survey Water-Resources Investigations Report 90-4162, p. 31-40.

Matthes, W.J., Sholar, C.J., and George, J.R., 1992, Quality-assurance plan for the analysis of fluvial sediment by laboratories of the U.S. Geological Survey: U.S. Geological Survey Open-File Report 91-467, $31 \mathrm{p}$.

Melcher, N.B., 1988, Computing stream discharge for stream-gaging stations in Iowa during periods of ice effect using index stations and regression equations, in International Symposium on Ice, 9th, Proceedings: Sapporo, Japan, 1988, v. 2, p. 293-303.

Melcher, N.B., Baebenroth, R.W., Detroy, M.G., Karsten, R.A., and Matthes, W.J., 1988, Water resources data, Iowa, water year 1987: U.S. Geological Survey Water-Data Report IA-87-1, 345 p.

Melcher, N.B., Detroy, M.G., Karsten, R.A., and Matthes, W.J., 1989, Water resources data, Iowa, water year 1988: U.S. Geological Survey Water-Data Report IA-88-1, 377 p.

Melcher, N.B., Detroy, M.G., Matthes, W.J., and Hansen, R.E., 1986, Water resources data, Iowa, water year 1985: U.S. Geological Survey Water-Data Report IA-85-1, 332 p. 
Melcher, N.B., Detroy, M.G., Matthes, W.J., and Karsten, R.A., 1987, Water resources data, Iowa, water year 1986: U.S. Geological Survey Water-Data Report IA-86-1, 333 p.

Melcher, N.B., and Walker, J.F., 1990, Evaluation of selected methods for determining streamflow during periods of ice effect: U.S. Geological Survey Open-File Report 90-554, 51 p.

Miller, V.E., Matthes, W.J., Detroy, M.G., and Hansen, R.E., 1985, Water resources data, Iowa, water year 1984: U.S. Geological Survey Water-Data Report IA-84-1, 263 p.

O’Connell, D.J., Liszewski, M.J., Lambert, R.B., and Matthes, W.J., 1989, Water resources data, Iowa, water year 1989: U.S. Geological Survey Water-Data Report IA-89-1, 399 p.

1990, Water resources data, Iowa, water year 1990: U.S. Geological Survey Water-Data Report IA-90-1, 421 p.

Onstad, C., Burkart, M.R., and Bubenzer, G. D., 1991, Agricultural research to improve water quality: Journal of Soil and Water Conservation, v. 46 , no. 3 , p. 184-188.

Ragone, S.E., Burkart, M.R., Thurman, E.M., and Perry, C.A., 1988, Herbicides in ground and surface water in the mid-continental United States--A research plan, in Annual Meeting of the Agricultural Research Institute, 37th, Proceedings: Washington, D.C., 1988, p. 55-67.

Runkle, D.L., [1986], Hydrology of the alluvial, buried channel, basal Pleistocene and Dakota aquifers in west-central Iowa: U.S. Geological Survey Water-Resources Investigations Report 85-4239, $111 \mathrm{p}$.

Runkle, D.L., Newman, J.L., and Shields, E.M., 1986, Permitted water use in Iowa: U.S. Geological Survey Open-File Report 86-302, 24 p.

Schulmeyer, P.M., 1991, Relation of selected water-quality constituents to river stage in the Cedar River, Iowa, in Mallard, G.E., and
Aronson, D.A., compilers, U.S. Geological Survey Toxics Substances Hydrology Program, Abstracts of the Technical Meeting, Monterey, California, 1991: U.S. Geological Survey Open-File Report 91-88, p. 18 .

1991, Relation of selected water-quality constituents to river stage in the Cedar River, Iowa, in Mallard, G.E., and Aronson, D.A., eds., U.S. Geological Survey Toxic Substances Hydrology Program, Proceedings of the Technical Meeting, Monterey, California, 1991: U.S. Geological Survey Water-Resources Investigations Report 91-4034, p. 227-231.

Soenksen, P.J., 1990, Automatic tracer-dilution method used for stage-discharge ratings and flood hydrographs on small Iowa streams: U.S. Geological Survey Water-Resources Investigations Report 89-4187, $45 \mathrm{p}$.

Soenksen, P.J., Eash, D.A., Hillaker, H.J., and Gordon, E.M., 1991, Iowa floods and droughts, in National water summary 1988-89--Hydrologic events and floods and droughts: U.S. Geological Survey Water-Supply Paper 2375, p. 279-286.

Squillace, P.J., and Engberg, R.A., 1988, Surface-water quality of the Cedar River basin, Iowa-Minnesota, with emphasis on the occurrence and transport of herbicides, May 1984 through November 1985: U.S. Geological Survey Water-Resources Investigations Report 88-4060, 81 p.

Squillace, P.J., Sandstrom, M.W., and Pereira, W.E., 1989, Herbicides in surface and ground water, Cedar River basin, Iowa and Minnesota, in Pederson, G.L., and Smith, M.M., compilers, U.S. Geological Survey Second National Symposium on Water Quality, Abstracts of the Technical Sessions, Orlando, Florida, 1989: U.S. Geological Survey Open-File Report 89-409, p. 95. 
Squillace P.J., and Thurman, E.M., 1989, Herbicide transport in the Cedar River, Iowa--Importance of hydrology and geochemistry in nonpoint-source contamination, in Mallard, G.E., and Ragone, S.E., eds., U.S. Geological Survey Toxic Substances Hydrology Program, Proceedings of the Technical Meeting, Phoenix, Arizona, 1988: U.S. Geological Survey Water-Resources Investigations Report 88-4200, p. 114.

1990, Source of atrazine and desethylatrazine in a selected reach of the Cedar River, Iowa during base flow conditions: EOS, Transactions of the American Geophysical Union, v. 71 , no. 43 , Oct. 23, 1990, p. 1331.

1991, Source of atrazine and desethylatrazine in a selected reach of the Cedar River, Iowa during base flow conditions: Iowa Ground Water Association, Oakdale Iowa, v. 3, no. 2, p. 7.

1992, Herbicide transport in the Cedar River, Minnesota and Iowa--Importance of hydrology and geochemistry in nonpoint-source contamination: Environmental Science and Technology, v. 26, no. 3, p. 538-545.

Squillace, P.J., Thurman, E.M., Fischer, E.E., and Soenksen, P.J., 1991a, Source of atrazine, desethylatrazine, and metolachlor in a selected reach of the Cedar River, Iowa, during base-flow conditions, in Mallard, G.E., and Aronson, D.A., compilers, U.S. Geological Survey Toxic Substances Hydrology Program, Abstracts of the Technical Meeting, Monterey, California, 1991: U.S. Geological Survey Open-File Report, 91-88, p. 11.

1991b, Source of atrazine and desethylatrazine in a river, during base flow, in Ritter, W.F., ed., Irrigation and Drainage, Proceedings of the 1991 National Conference of the American Society of Civil Engineers: Honolulu, Hawaii, 1991, p. 253-259.
1991, Source of atrazine, desethylatrazine, and metolachlor in a selected reach of the Cedar River, Iowa, during base-flow conditions, in Mallard, G.E., and Aronson, D.A., eds., U.S. Geological Survey Toxics Substances Hydrology Program, Proceedings of the Technical Meeting, Monterey, California, 1991: U.S. Geological Survey Water-Resources Investigations Report 91-4034, p. 189-194.

Thamke, J.N., 1988, Iowa water use and supply, in National Water Summary 1987-Hydrologic events and water supply and use: U.S. Geological Survey Water-Supply Paper 2350, p. 251-258.

Thamke, J.N., and Clark, M.L., 1988, Populations affected by pesticides in public ground-water supplies in Iowa, in Symposium on Water-Use Data for Water Resources Management, Proceedings, Tucson, Arizona, 1988: American Water Resources Association Technical Publication Series TPS-88-2, p. 381-387.

Thurman, E.M., Goolsby, D.A., Meyer, M.T., and Kolpin, D.W., 1991, Herbicides in surface waters of the midwestern United States--Effects of the spring flush: Environmental Science and Technology, v. 25 , no. 10 , p. 1794-1796.

Thurman, E.M., Squillace, P.J., Strezpek, K.M., and Garcia, L., 1988, Intelligent data analyzer--Herbicide transport in the Cedar River, Iowa, in Preprints of papers presented at the 196th American Chemical Society National Meeting, v. 26, no. 2, Division of Environmental Chemistry, American Chemical Society, Washington, D.C., 1988: p. 13-15.

For a listing of reports published by the Iowa District, U.S. Geological Survey, prior to fiscal year 1984 see:

Karsten, R.A., 1985, Iowa--A summary of water resources activities of the United States Department of the Interior Geological Survey: Iowa City, Iowa, U.S. Geological Survey Report, 46 p. 